Contract No. and Disclaimer:

This manuscript has been authored by Savannah River Nuclear Solutions, LLC under Contract No. DE-AC09-08SR22470 with the U.S. Department of Energy. The United States Government retains and the publisher, by accepting this article for publication, acknowledges that the United States Government retains a non-exclusive, paid-up, irrevocable, worldwide license to publish or reproduce the published form of this work, or allow others to do so, for United States Government purposes. 


\title{
Numerical Modeling of Contaminant Transport in Fractured Porous Me- dia using Mixed Finite Element and Finite Volume Methods
}

\author{
Chen Dong ${ }^{1}$, Shuyu Sun ${ }^{1, *}$, Glenn A. Taylor ${ }^{2}$ \\ ${ }^{1}$ Department of Mathematical Sciences, Clemson University \\ Clemson, SC 29634-0975, USA \\ ${ }^{2}$ Savannah River National Laboratory \\ Aiken, SC 29808, USA
}

\begin{abstract}
A mathematical model for contaminant species passing through fractured porous media is presented. In the numerical model, we combine two locally conservative methods, i.e. mixed finite element (MFE) and the finite volume methods. Adaptive triangle mesh is used for effective treatment of the fractures. A hybrid MFE method is employed to provide an accurate approximation of velocities field for both the fractures and matrix which are crucial to the convection part of the transport equation. The finite volume method and the standard MFE method are used to approximate the convection and dispersion terms respectively. The model is used to investigate the interaction of adsorption with transport and to extract information on effective adsorption distribution coefficients. Numerical examples in different fractured media illustrate the robustness and efficiency of the proposed numerical model.
\end{abstract}

Keywords: mixed finite element method, finite volume method, adaptive triangle mesh, fractured porous medium, flow transportation, simulation.

\section{Introduction}

It is well known that fractures play an important role in flow and transport processes through saturated and unsaturated geologic media. By fracture, we mean the opening in the media that is small in one direction (the fracture aperture) and relatively large in two directions along the plane of fracture. A fracture to be modeled in this paper could be any local separation or discontinuity plane (such as joint or fault) in a geologic formation; it could also be the smallscale cracks in concrete barriers. Because fracture permeability is generally much greater than cementitious matrix permeability, fracture networks have the potential for being highly effective pathways for conducting fluid containing contaminant species.

Since the fracture geometry and connectivity of fractured rock is complex, flow behavior through saturated and unsaturated fractures is difficult to characterize for a given site (Bodvarsson et al., 2002). Even with the significant progress made in the last two decades, many aspects of flow and transport processes in fractured rocks are currently still poorly understood. The main challenge arises from technical difficulties in observing details of flow processes and accurately describing such phenomena within fractured rocks. However, flow processes occurring at this scale are crucial to many field-scale applications.

In recent years, through field studies as well as mathematical simulations, considerable progress has been made in understanding convection-dominated processes within fractured matrix. Glass et al. (1996) demonstrated that the main flow mechanism for a vertical fractured is fingering which results from gravitational instability and aperture heterogeneities. Tokunaga and Wan (1997) showed that film flow could be an important mechanism at low fracture saturations. In their laboratory experiments, Su et al. (1999) demonstrated intermittent flow behavior not con- 
sidered by classical theory. However how to incorporate this small-scale mechanism into fieldscale models remains a challenge. Bodvarsson et al., (2002) used an approach to investigate the development of flow focusing and discrete paths that may occur through fractures. To quantify flow-focusing behavior, their stochastic fracture-continuum models incorporated fracture data measured from the welded tuffs to study flow-allocation mechanisms and patterns.

A number of approaches are available in the literature to model flow and transport in saturated and unsaturated fractured matrix. In general, these modeling approaches fall in to one of the two categories: the continuum approach and the discrete fracture network approach. Excellent reviews on these approaches which have been developed and used in different fields (including oil reservoir engineering, groundwater hydrology, geothermal engineering, and soil physics), which can be found in Bear et al.(1993), National Research Council (1996), and Pruess et al. (1999). The dual-porosity model (Warren and Root, 1963; Kazemi, 1969; Thomas et al., 1983; Arbogast et al., 1990) is widely used in the flow simulators in fractured media. This model is numerically efficient since computations are performed separately for the matrix and for in the fracture-equivalent media. However, because appropriate transfer functions are not well established, the model is inadequate to describe the compositional effects in fractured media. Another limitation is the lack of taking into account the discrete fractures specifically, since this model assumes the medium to be described by a dense connected fractured network. One can also use the single-porosity model with explicit grid blocks to describe the fractures in the same way as the matrix is described. An alternative is the discrete fracture model, which can be considered as a simplification of the single-porosity model. Assuming that the fracture aperture is small compared to the matrix scale, fractures are represented by (n-1)-dimensional elements in an $n$ dimensional domain (Noorishad and Mehran,1982; Baca et al., 1984; Granet et al., 1998).

Recent works in fractured porous media simulation are more relevant to our project. Kim and Deo (1999, 2000), Bastian et al. (2000), Karimi-Fard and Firoozabadi (2003) have studied immiscible fluid flow excellently and extensively. H. Hoteit and A. Firoozabadi (2005) demonstrated the possibility of modeling multi-component compressible flow in discrete fractured media. The reliability and efficiency of this approach are conditional to two essential approximations, the matrix fracture and the fracture-fracture fluxes. The pressure in a fracture element is assumed to be equal to the pressure in the surrounding matrix elements, based on the cross-flow equilibrium concept.

Barriers which this paper considers consisting of porous media, i.e. cement, grout, rock, etc., are important for the sequestration of radioactive waste. Contaminant transport through these barriers is typically calculated using a bulk material property known as the distribution coefficient, $\mathrm{K}_{\mathrm{d}}$, which represent the distribution of contaminant between the solid and liquid phases. The amount of solute adsorbed on the solid part of the porous media is expressed per unit mass of the bulk medium in a dry state. A unit mass of the porous medium is used as a reference quantity. The effect of the $K_{d}$ is to show a retardation in the breakthrough curve of a contaminant. The retardation of the contaminant front is relative to the bulk water. A more mechanistic, but more difficult to implement, approach would be to use a unit surface area of the porous medium as the reference quantity. Adsorption reactions are more closely related to surface area than to mass so using surface area would be an improvement in the definition of the distribution coefficient. Retardation is extremely important in the analyses and design of barriers to radioactive waste migration. Regulations and laws which govern permissible releases from shallow land burial of low level radioactive wastes typically have some period of performance. That 
is, at some time in the future, say 1,000 or 10,000 years, the peak release from a burial facility must be below some performance measure.

In this work, we employ discrete fracture model to describe flow and transport processes in fractured media. Unlike the classical discrete fracture model where (n-1)-dimensional elements are used to represent the cracks, our discrete fracture model still use physically meaningful ndimensional elements. We address the numerical difficulty arising from the fracture by using mesh adaptation rather than by using ( $n-1)$-dimensional elements. A numerical scheme based on mixed finite element (MFE) method is developed to approximate the second-order partial derivate terms in the flow and transport equations. The convection term in the transport is treated using an upwind finite volume method (FVM), and the adsorption is modeled via effective porosity.

This paper is organized as follows: First, the differential equations describing the contaminant diffusion and transport in the fractured cementitious matrix are presented. Second, a numerical model in saturated fractured media is described. We discuss in detail various components of our numerical approach, which include the MFE method for the flow equation, the combined FVMMFE method for the transport equation and the numerical discretization in time. We then present numerical examples in fractured media with various fracture distributions. For each example, we provide and discuss simulated concentration profiles at different times, together with pressure and velocity fields. Finally, we numerically carry out a sensitivity analysis of parameters in our model and investigate the relationship between the intrinsic $K_{d}$ value and effective $\mathrm{K}_{\mathrm{d}}$ value which will help to design barriers that is protective of the public and environment while at the same time not being so over-designed as to be prohibitively expensive.

\section{Mathematical model}

Modeling equations for contaminant species through cracked porous media consists of two coupled differential equations, one is the flow equation and the other is reactive transport equation.

\section{Flow Equation:}

The flow equation is obtained from the conservation of total fluid volume and Darcy's law.

$$
-\nabla \cdot(\mathrm{K} \nabla p) \equiv \nabla \cdot \mathbf{u}=0, \quad x \in \Omega .
$$

Here $\mathrm{K}$ is the conductivity defined by

$$
\mathrm{K}=\frac{\text { permeability }}{\text { viscosity }} \text {. }
$$

The unknowns are $p$ (the pressure in the fluid mixture) and $\mathbf{u}$ (the Darcy velocity of the mixture, i.e. the volume of fluid flowing cross a unit cross-section per unit time). The conductivity $K$ is assumed to be uniformly symmetric positive definite and bounded.

We assume our domain $\Omega$ is a polygonal and bounded in $R^{d}(d=1,2$, or 3 ) with boundary $\partial \Omega=\Gamma_{D} \cup \Gamma_{N}$. Here $\Gamma_{D}$ is the Dirichlet boundary and $\Gamma_{N}$ is the Neumann boundary. The boundary conditions are taken as: 


$$
\begin{array}{ll}
p=p_{B}, & x \in \Gamma_{D}, \\
\mathbf{u} \cdot \mathbf{n}=u_{B}, & x \in \Gamma_{N} .
\end{array}
$$

\section{Reactive Transport Equations:}

The reactive equation system is obtained from the conservation of considered contaminant species. The contaminant concentrations in the fluid and in the solid as well as their relation can be described by

$$
\left\{\begin{array}{l}
\frac{\partial \phi c}{\partial t}+\nabla \cdot(\mathbf{u} c-D(\mathbf{u}) \nabla c)=r\left(c, c_{s}\right), \\
\frac{\partial\left(\rho c_{s}\right)}{\partial t}=-r\left(c, c_{s}\right), \\
c_{s}=K_{d} c .
\end{array} \quad(x, t) \in \Omega \times(0, T] .\right.
$$

Here the unknown $c$ is the concentration of the interested species within the fluid (i.e. the amount of the species per unit volume of the fluid mixture) and $c_{s}$ the concentration of the interested species in the solid. $T$ is the final simulation time. The parameter $K_{d}$ is the partitioning coefficient of the considered species between the fluid and the rock.

Summation of the two concentration equations above (one in fluid and another one in solid) yields:

$$
\frac{\partial \phi^{e f f} c}{\partial t}+\nabla \cdot(\mathbf{u} c-D(\mathbf{u}) \nabla c)=0
$$

Where $\phi^{e f f}=\phi+\rho K_{d}$ is the effective porosity and it is calculated separately for the matrix and for the fractures:

In the matrix: $\quad \phi=\phi_{\text {matrix }}, \phi^{\text {eff }}=\phi+\rho K_{d}$

In the fracture: $\quad \phi=1, K_{d}=0, \phi^{e f f}=\phi=1.0$

We impose the following boundary conditions:

$$
\begin{array}{ll}
(\mathbf{u} c-D(\mathbf{u}) \nabla c) \cdot \mathbf{n}=c_{B} \mathbf{u} \cdot \mathbf{n}, & x \in \Gamma_{\text {inflow }}, \\
-D(\mathbf{u}) \nabla c \cdot \mathbf{n}=0, & x \in \Gamma_{\text {outflow }} .
\end{array}
$$

Here $\Gamma_{\text {inflow }}$ is the inflow boundary and $\Gamma_{\text {outflow }}$ is the outflow boundary, i.e,

$$
\begin{aligned}
& \Gamma_{\text {inflow }}=\left\{x \in \partial \Omega: \mathbf{u}(x) \cdot \mathbf{n}_{\partial \Omega}<0\right\}, \\
& \Gamma_{\text {oufflow }}=\left\{x \in \partial \Omega: \mathbf{u}(x) \cdot \mathbf{n}_{\partial \Omega} \geq 0\right\} .
\end{aligned}
$$

We specify an initial concentration:

$$
c(x, 0)=c_{0}(x) \text {. }
$$


The porosity $\phi$ is assumed to be time-independent, uniformly bounded above and below by positive numbers. The dispersion-diffusion tensor $\mathrm{D}(\mathbf{u})$ has contributions from molecular diffusion and mechanical dispersion, and can be calculated by

$$
D(\mathbf{u})=d_{m} I+|\mathbf{u}|\left\{\alpha_{l} E(\mathbf{u})+\alpha_{t}(I-E(\mathbf{u}))\right\},
$$

where $\mathrm{E}(\mathbf{u})$ is the tensor that projects onto the $\mathbf{u}$ direction, whose $(i, j)$ component is $(\mathrm{E}(\mathbf{u}))_{\mathrm{ij}}=\frac{\mathrm{u}_{\mathrm{i}} \mathrm{u}_{\mathrm{j}}}{|\mathrm{u}|^{2}} ; d_{m}$ is the molecular diffusivity and is assumed to be strictly positive; $\alpha_{1}$ and $\alpha_{t}$ are the longitudinal and the transverse dispersivities, respectively, and are assumed to be nonnegative.

\section{Numerical Algorithms:}

In this work, the system contains two parts: a flow equation involving the pressure and Darcy velocity, and a contaminant species transport equation for describing the evolution of concentrations. We first generate a triangular mesh containing fractures represented by long and thin rectangles consisting of small triangles. Based on this triangular mesh, we solve the flow equation by a mixed finite element method (MFE); then we solve the reactive transport equation semi-implicitly (explicitly for convection and implicitly for diffusion and adsorption) in time by using the combination of a finite volume method (FVM) and MFE method.

\section{Mesh generation for fractured media}

Creating a mesh is the first step in our algorithm. Since the fractures are distributed randomly in the domain, not just vertical to the boundaries, the triangular mesh is better to fit the fractured media than the rectangular mesh which is easier to generate. The mesh quality is important here since a low mesh quality will significantly affect the efficiency and accuracy of the algorithm. There are several mesh generation methods including the TRIANGLE (J. Shewchuk, 1996), DISTMESH (P. -O. Persson and G.Strang, 2004) and MESHGEN (L. Ju, 2007). Although all of these methods yield good results in most cases, the two methods later are proved to have better mesh quality than the first one (H. Nguyen, J. Burkardt, M. Gunzburger, L. Ju, Y. Saka, 2009). In this paper, we modify the code of DISTMESH for the 2D fractured media. At first, several fractures are randomly distributed in the $0.6 \mathrm{~m} \times 0.6 \mathrm{~m}$ rectangular domain. The length of each fracture is randomly chosen from $0.02 \mathrm{~m}$ to $0.2 \mathrm{~m}$. The angle between each fracture and the lower boundary is from 0 to $2 \pi$. The aperture of fractures is set to $0.3 \mathrm{~mm}$ (Liu and Bodvarsson 2001). We constrain the center of each fracture to lie in the region $0.5 \mathrm{~m} \times 0.5 \mathrm{~m}$ so that there is no fracture out of the domain in the worst case. Next, we generate conforming triangular mesh to fit these fractures. We refine the triangular mesh with small triangles near fractures for it is where several parameters including concentration and velocity of contaminant species would change significantly during the simulation. In our model, each fracture is represented in two-dimension, which means all fractures consist of triangles but have much lower porosity. We keep the mesh fixed during our simulation for simplicity.

\section{Mixed Finite Element method:}

We apply a mixed finite element method for the treatment of the flow equation. MFE methods (Brezzi \& Fortin, 1991) are based on a variational principle expressing an equilibrium or saddle point condition that can be satisfied locally on each finite element. The MFE formulation for the flow equation involves solving for both the scalar variable (pressure) and the flux vector (total velocity). Approximating spaces for the MFE method can be chosen to satisfy three important properties: local mass conservation, flux continuity, and the same order of convergence (and in 
some cases superconvergence) for both the scalar variable and the flux (Ewing, Lazarov, \& Wang, 1991). Because of the finite element setting, MFE can readily accommodate full permeability tensors. By using a mixed formulation, the MFE method is more accurate in flux calculation than the conventional finite volume and finite element methods.

Let $(., .)_{D}$ denote the $L^{2}(D)$ inner product over a domain $D \subset \mathbb{R}^{d}, d=1,2,3$ for scalar functions and the $\left(L^{2}(D)\right)^{d}$ inner product for vector functions. We use (.,.), when $D=\Omega$. And the $L^{P}(D)$ norm for a scalar function or the $\left(L^{P}(D)\right)^{d}$ norm for a vector function is denoted by

$$
\|\cdot\|_{L^{p}(D)}, 1 \leq p \leq \infty
$$

We define the following standard spaces:

$$
\begin{aligned}
& W=L^{2}(\Omega) \\
& V=H(\operatorname{div} ; \Omega)=\left\{\mathbf{v} \in\left(L^{2}(\Omega)\right)^{d}: \nabla \cdot \mathbf{v} \in L^{2}(\Omega)\right\} \\
& V^{0}=\{\mathbf{v} \in H(\operatorname{div} ; \Omega): \mathbf{v} \cdot \mathbf{n}=0 \text { on } \partial \Omega\} \\
& V_{N}^{0}=\left\{\mathbf{v} \in H(\operatorname{div} ; \Omega): \mathbf{v} \cdot \mathbf{n}=0 \text { on } \Gamma_{N}\right\}
\end{aligned}
$$

\section{MFE for Flow equation:}

\section{Weak formulation}

The weak formulation of the flow equation is to find $\mathbf{u} \in V_{N}^{0}+E\left(u_{B}\right), p \in W$ such that:

$$
\begin{cases}\left(\mathrm{K}^{-1} \mathbf{u}, \mathbf{v}\right)-(\nabla \cdot \mathbf{v}, p)=-\int_{\Gamma_{D}} p_{B} \mathbf{v} \cdot \mathbf{n} d s, & \forall \mathbf{v} \in V_{N}^{0}, t \in(0, T], \\ (\nabla \cdot \mathbf{u}, w)=(0, w), & \forall w \in W, t \in(0, T],\end{cases}
$$

Here $E\left(u_{B}\right)$ is the velocity extension such that its normal component agrees with $u_{B}$ on $\Gamma_{N}$.

\section{MFE scheme}

We now employ the Raviart-Thomas (RT) space [Raviart,R.A. 1977] to approximate the Darcy velocity.

For a two-dimensional triangular element, the $r$-th order RT space is defined by

$V_{h}(K)=\left(P_{r}(K)\right)^{2} \oplus\left(\left(x_{1}, x_{2}\right) P_{r}(K)\right)$

$W_{h}(K)=P_{r}(K)$

Restricted to the element $K, P_{r}(K)$ is the space of polynomials degree less than or equal to $r$. In our numerical examples below we use $\mathrm{RT}_{0}$ space. The MFE method for approximating the flow equation is to find $p_{h}(., t) \in W_{h}$ and $\mathbf{u}_{h}(., t) \in V_{h}^{0}=V^{0} \cap V_{h}+E_{h}\left(u_{B}\right)$, such that 


$$
\begin{cases}\left(\mathrm{K}^{-1} \mathbf{u}_{h}, \mathbf{v}\right)-\left(\nabla \cdot \mathbf{v}, p_{h}\right)=-\int_{\Gamma_{D}} p_{B} \mathbf{v} \cdot \mathbf{n} d s, & \forall \mathbf{v} \in V_{h}, \\ \left(\nabla \cdot \mathbf{u}_{h}, w\right)=(0, w), & \forall w \in W_{h} .\end{cases}
$$

Since the original MFE formulation leads to a saddle point problem for elliptic equations, we use the Mixed-Hybrid algorithms [Brezzi and Fortin, 1991; Chavent and Roberts, 1991] for the pressure equation. The main idea is to add unknowns which represent the edge pressure averages, such that the reduced linear system we solve includes a symmetric and positive definite matrix and thus it has advantages in iterative linear solvers.

\section{Matrix formulation}

On the basis of $\mathrm{RT}_{0}$ space, the $\mathbf{u}_{h}$ in the linear system (2) can be expressed as

$$
\mathbf{u}_{h}=\sum_{E \in \partial K} u_{K, E} v_{K, E}
$$

Where $v_{K, E}$ is a $\mathrm{RT}_{0}$ basis function, $u_{K, E}$ is the total flux across an edge $E$. For convenience, we first consider a single element $\mathrm{K}$. Substituting $\mathbf{v}$ with the test function $v_{K, E}$, the total flux can be expressed as

$$
A_{K} U_{K}=p_{K} e-P_{K E}
$$

Where

$$
\begin{aligned}
& A_{K}=\left[\left(A_{K}\right)_{E, E^{\prime}}\right]_{E, E^{\prime} \in \partial K}, \quad\left(A_{K}\right)_{E, E^{\prime}}=\int_{K} v_{K, E} \mathrm{~K}^{-1} v_{K, E^{\prime}}, \\
& U_{K}=\left[u_{K, E}\right]_{E \in \partial K}, \quad e=[1]_{E \in \partial K}, \quad P_{K, E}=\left[\bar{p}_{K, E}\right]_{E \in \partial K} .
\end{aligned}
$$

Thus equation (4) expresses the flues $u_{K, E}$ through each edge as a function of the cell pressure average $p_{K}$ and edge pressure average $\bar{p}_{K, E}$,

$$
u_{K, E}=\alpha_{K, E} p_{K}-\sum_{E^{\prime} \in \partial K} \beta_{K, E, E^{\prime}} \bar{p}_{K, E^{\prime}}
$$

where, $\alpha_{K, E}=\sum_{E^{\prime} \in \partial K}\left(A_{K}^{-1}\right)_{E, E^{\prime}}, \beta_{K, E, E^{\prime}}=\left(A_{K}^{-1}\right)_{E, E^{\prime}}$.

In the second equation in linear system (2), $w \in W_{h}(K)=P_{0}(K)$. Together with equation (3), we have

$$
B_{K} U_{K}=0
$$

where 
$B_{K}=\left[\left(B_{K}\right)_{K, E}\right], \quad\left(B_{K}\right)_{K, E}=\int_{K}\left(\nabla \cdot v_{K, E}\right) w_{K}, w_{K}=1$

Equations (4) and (5) lead to

$$
p_{K} B_{K} A_{K}^{-1} e-B_{K} A_{K}^{-1} P_{K E}=0
$$

Thus

$$
p_{K}=\left(B_{K} A_{K}^{-1} e\right)^{-1} B_{K} A_{K}^{-1} P_{K E}
$$

The continuity of the fluxes across the inter-element boundaries provides

$$
u_{K, E}=\left\{\begin{array}{lll}
-u_{K, E^{\prime}} & \text { if } & E=K \cap K^{\prime}, \\
u_{E}^{N} & \text { if } & E \in \Gamma_{N} .
\end{array}\right.
$$

Then equations (4), (7) and (8) lead to the following algebraic linear system with main unknowns, the pressure edge averages in $P_{E}$.

$$
A_{T} P_{E}=V
$$

where

$$
\begin{aligned}
& A_{T}=Q^{T} R-M, \\
& Q=\left[Q_{K, E}\right]_{N_{K}, N_{E}}, \quad Q_{K, E}=\alpha_{K, E}, \quad E \in \partial K, \\
& R=\left[R_{K}\right]_{N_{K}}, R_{K}=\left(B_{K} A_{K}^{-1} e\right)^{-1} B_{K} A_{K}^{-1}, \\
& M=\left[M_{E, E^{\prime}}\right]_{N_{E}, N_{E}}, \quad M_{E, E^{\prime}}=\sum_{E, E^{\prime} \subset \partial K} \beta_{K, E, E^{\prime}}, \mathrm{E} \notin \Gamma_{D},
\end{aligned}
$$

The $N_{K}$ denotes the number of cells in the mesh and $N_{E}$ is the number of edges in the mesh not belonging to $\Gamma_{D}$. V is a vector of size $N_{E}$ which represents the boundary conditions.

\section{MFE and FVM for reactive transport equation:}

\section{Weak formulation}

The weak formulation of transport equation is to find the concentration solution $c \in W$ and the diffusive flux solution $\mathbf{v} \in V^{0}$ such that: 


$$
\begin{cases}\left(\frac{\partial \phi^{e f f} c}{\partial t}, w\right)+(\nabla \cdot \mathbf{v}, w)+\sum_{K} \int_{\partial K} w \mathbf{u} c^{*} \cdot \mathbf{n} d s-\sum_{K}\left(c^{*} \mathbf{u}, \nabla w\right)=0, & \forall w \in W, t \in(0, T], \\ \left(-D^{-1} \mathbf{v}, \hat{\mathbf{v}}\right)+(c, \nabla \cdot \hat{\mathbf{v}})=0, & \forall \hat{\mathbf{v}} \in V^{0}, t \in(0, T], \\ (c, w)=\left(c_{0}, w\right), & \forall w \in W, t=0 .\end{cases}
$$

Here $c^{*}$ denotes the upwind value of the concentration on an edge.

\section{MFE scheme}

Now the continuous-in-time MFE method for approximating the transport equation is to find $c_{h} \in W_{h}, \mathbf{v}_{h} \in V_{h}^{0}$ such that:

$$
\begin{cases}\left(\frac{\partial \phi^{e f f} c_{h}}{\partial t}, w\right)+\left(\nabla \cdot \mathbf{v}_{h}, w\right)+\sum_{K} \int_{\partial K} w \mathbf{u} c_{h}^{*} \cdot \mathbf{n} d s-\sum_{K}\left(c_{h}^{*} \mathbf{u}, \nabla w\right)=0, & \forall w \in W_{h}, t \in(0, T], \\ \left(-D^{-1} \mathbf{v}_{h}, \hat{\mathbf{v}}\right)+\left(c_{h}, \nabla \cdot \hat{\mathbf{v}}\right)=0, & \forall \hat{\mathbf{v}} \in V_{h}^{0}, t \in(0, T], \\ \left(c_{h}, w\right)=\left(c_{0}, w\right), & \forall w \in W_{h}, t=0 .\end{cases}
$$

Now for the transport equation, we consider a fully discretized algorithm. We partition the simulation time $[0, T]$ into $m$ subintervals: $0=t_{0}<t_{1}<\cdots<t_{m-1}<t_{m}=T$. We let $\Delta t_{k}=t_{k}-t_{k-1}$, $\Delta t=\max \Delta t_{k}$. Assuming that there exists a constant $C$ satisfying that $\Delta t \leq C \min \Delta t_{k}$, the transport equation can be solved by semi-implicit Euler method in time and the combined FVMMFE method in space. The fully discretized approximation is to find $c_{h, k} \in W_{h}$ and $\mathbf{v}_{h, k} \in V_{h}^{0}$, for $k=0,1,2, \ldots, m$, such that

$$
\begin{cases}\left(\frac{\phi^{e f f} c_{h, k}-\phi^{e f f} c_{h, k-1}}{\Delta t}, w\right)+\left(\nabla \cdot \mathbf{v}_{h, k}, w\right)+\sum_{K} \int_{\partial K} w \mathbf{u} c_{h, k-1}^{*} \cdot \mathbf{n} d s-\sum_{K}\left(c_{h, k-1}^{*} \mathbf{u}, \nabla w\right)=0, & \forall w \in W_{h}, t \in(0, T], \\ \left(-D^{-1} \mathbf{v}_{h, k}, \hat{\mathbf{v}}\right)+\left(c_{h, k}, \nabla \cdot \hat{\mathbf{v}}\right)=0, & \forall \hat{\mathbf{v}} \in V_{h}^{0}, t \in(0, T], \\ \left(c_{h, 0}, w\right)=\left(c_{0}, w\right), & \forall w \in W_{h}, t=0 .\end{cases}
$$

\section{Matrix formulation}

We use standard MFE algorithm to solve this reactive transport equation. For the second equation in the linear system (10), similar to the algorithm solving the flow equation, we have that on the basis of $\mathrm{RT}_{0}$ space, the $\mathbf{v}_{h}$ in linear system (2) can be expressed as

$$
\mathbf{v}_{h}=\sum_{E \in \partial K} \tilde{v}_{K, E} \hat{v}_{K, E}
$$


Where $\hat{v}_{K, E}$ is a $\mathrm{RT}_{0}$ basis function, $\tilde{v}_{K, E}$ is the total diffusive flux across an edge $E$. Substituting $\hat{\mathbf{v}}$ with the test function $\hat{v}_{K, E}$ and integrating by parts, then the total diffusive flux can be expressed as

$$
A_{K}^{\text {conc }} \tilde{V}_{K}=c_{K} B_{K}^{c o n c}
$$

Where

$$
\begin{aligned}
& A_{K}^{c o n c}=\left[\left(A_{K}^{c o n c}\right)_{E, E^{\prime}}\right]_{E, E^{\prime} \in \partial K}, \quad\left(A_{K}^{c o n c}\right)_{E, E^{\prime}}=\int_{K} \hat{v}_{K, E} D^{-1} \hat{v}_{K, E^{\prime}}, \\
& \tilde{V}_{K}=\left[\tilde{v}_{K, E}\right]_{E \in \partial K}, \\
& B_{K}^{c o n c}=\left[\left(B_{K}^{c o n c}\right)_{K, E}\right], \quad\left(B_{K}^{c o n c}\right)_{K, E}=\int_{K}\left(\nabla \cdot \hat{v}_{K, E}\right) w_{K}, \quad w_{K}=1 .
\end{aligned}
$$

For the diffusion part in the first equation in linear system (10),

$$
\left(\frac{\partial \phi^{e f f} c_{h}}{\partial t}, w\right)+\left(\nabla \cdot \mathbf{v}_{h}, w\right)=0
$$

We have

$$
M_{K}^{\text {conc }} \frac{d c_{K}}{d t}+\left(B_{K}^{\text {conc }}\right)^{T} \tilde{V}_{K}=0
$$

Where

$$
M_{K}^{c o n c}=\left[\left(M_{K}^{c o n c}\right)_{K}\right], \quad\left(M_{K}^{c o n c}\right)_{K}=\int_{K} \phi^{e f f} w_{K} \cdot w_{K}, \quad w_{K}=1
$$

Then equations (14) and (16) lead to the following algebraic linear system with main unknowns, the concentration cell averages $C$ and the diffusive flux $\tilde{V}$.

$$
M_{T} \frac{d Y}{d t}+\tilde{A}_{T} Y=0,
$$

where

$$
\begin{aligned}
& M_{T}=\left(\begin{array}{cc}
0 & 0 \\
0 & M^{c o n c}
\end{array}\right), M^{c o n c}=\operatorname{diag}\left(M_{K}^{\text {conc }}\right)_{N_{K}}, \\
& Y=\left(\begin{array}{l}
\tilde{V} \\
C
\end{array}\right), \tilde{A}_{T}=\left(\begin{array}{cc}
A^{c o n c} & -B^{c o n c} \\
\left(B^{c o n c}\right)^{T} & 0
\end{array}\right), A^{\text {conc }}=\operatorname{diag}\left(A_{K}^{c o n c}\right)_{N_{K}}, B^{c o n c}=\left[B_{K}^{c o n c}\right]_{N_{K}} .
\end{aligned}
$$

For the advection part in the first equation in linear system (10), 


$$
\left(\frac{\partial \phi^{e f f} c}{\partial t}, w\right)+\sum_{K} \int_{\partial K} w \mathbf{u} c^{*} \cdot \mathbf{n} d s-\sum_{K}\left(c^{*} \mathbf{u}, \nabla w\right)=0
$$

Since the space we consider is $\mathrm{RT}_{0}$, thus $\nabla w=0$. Equation (18) can be simplified as

$$
\left(\frac{\partial \phi^{e f f} c}{\partial t}, w\right)+\sum_{K} \int_{\partial K} w \mathbf{u} c^{*} \cdot \mathbf{n} d s=0
$$

The velocity vector $\mathbf{u}_{r}$ across each edge can be derived in equation (4). Let

$$
\bar{u}_{r}=\mathbf{u}_{r} \cdot \mathbf{n}_{r}, r=1,2, \ldots, N_{E} .
$$

$\mathbf{n}_{r}$ is the unit normal vector of each edge. To find the upwind value of the concentration on each edge, let $G^{+}$denote the edge-element signed adjacency matrices where the entry equals 1 if $\bar{u}_{r}>0$ for each edge $r$, on contrary, the entry of $G^{-}$equals -1 if $\bar{u}_{r}<0$. Then the upwind concentration on edges can be expressed as $G^{+} C$ or $G^{-} C$. Let $\overline{\mathbf{u}}=\left[\bar{u}_{r}\right]_{N_{E}}, \overline{\mathbf{u}}^{+}=\max (\overline{\mathbf{u}}, 0)$, $\overline{\mathbf{u}}^{-}=\min (\overline{\mathbf{u}}, 0)$, taking account of the boundary condition, then the flux across edges can be expressed as

$$
\mathbf{f}=\operatorname{diag}\left(\overline{\mathbf{u}}^{+}\right) G^{+} C+\operatorname{diag}\left(\overline{\mathbf{u}}^{-}\right)\left(G^{-} C+B C_{B}\right) .
$$

Here $B$ is the edge-boundary adjacency matrix and $C_{B}$ is the concentration on inflow boundary. Then the total divergence amount is

$$
G^{T} \mathbf{f}=G^{T}\left(\operatorname{diag}\left(\overline{\mathbf{u}}^{+}\right) G^{+}+\operatorname{diag}\left(\overline{\mathbf{u}}^{-}\right) G^{-}\right) C+G^{T} \operatorname{diag}\left(\overline{\mathbf{u}}^{-}\right) B C_{B},
$$

Here $G$ is the edge-element adjacency matrix, $G=G^{+}-G^{-}$. Equation (20) leads to an algebraic linear system

$$
M^{\text {conc }} \frac{d C}{d t}+\hat{A} C=\hat{b}
$$

Where

$M^{\text {conc }}=\operatorname{diag}\left(M_{K}^{\text {conc }}\right)_{N_{K}}$ denoted in equation (16),

$\hat{A}=G^{T}\left(\operatorname{diag}\left(\overline{\mathbf{u}}^{+}\right) G^{+}+\operatorname{diag}\left(\overline{\mathbf{u}}^{-}\right) G^{-}\right)$,

$\hat{b}=-G^{T} \operatorname{diag}\left(\overline{\mathbf{u}}^{-}\right) B C_{B}$. 
The equation (17) and equation (21) lead to the final algebraic linear system for transport equation,

$$
M_{T} \frac{d Y}{d t}+\tilde{A}_{T} Y+\hat{A}_{T} Y=b
$$

Where

$$
\hat{A}_{T}=\left(\begin{array}{ll}
0 & 0 \\
0 & \hat{A}
\end{array}\right), b=\left(\begin{array}{l}
0 \\
\hat{b}
\end{array}\right) .
$$

For equation (22), we can easily employ the backward Euler method and treating equation (17) implicitly and equation (21) explicitly,

$$
M_{T} \frac{Y_{k+1}-Y_{k}}{\Delta t}+\tilde{A}_{T} Y_{k+1}+\hat{A}_{T} Y_{k}=b .
$$

The trapezoid methods, high order Runge-Kutta methods can also be employed in solving ODE equation (22).

\section{Numerical Results:}

\subsection{Simulation Examples}

All numerical examples presented in this paper are carried out on a computational domain of $(0$, $0.6 \mathrm{~m}) \times(0,0.6 \mathrm{~m})$. We partition a $(0,0.6 \mathrm{~m}) \times(0,0.6 \mathrm{~m})$ rectangular domain with random fractures into non-uniform and adaptive triangular mesh. We apply densely refined triangles to the area close to fractures. All the results are for non-uniform conforming grids and standard parameters.

We present seven numerical examples for contaminant species passing through 2D porous media on the domain of $(0,0.6 \mathrm{~m}) \times(0,0.6 \mathrm{~m})$. Example 1 is the base case to be compared with other six examples. In this base case, we use a set of standard parameters arising in typical applications in SRNL (see Table 1). In Examples 2 and 3, we manually set the fractures with different lengths and distributions, but the fracture does not extend onto the boundary. Example 4 is the case that the crack starts at one boundary but does not penetrate the medium all the way to the other end. Examples 5 and 6 simulate the fracture networks that extend onto both inflow and outflow boundaries. Examples 7 represent a more realistic scenario where we randomly generated different number of fractures in the domain to investigate the effect of fracture density. In all examples, no-flow boundary conditions apply to the top $(y=0.6 \mathrm{~m})$ and bottom boundaries $(y=0 \mathrm{~m})$. We specify a constant pressure of 0 (gauge pressure against a reference pressure) on the right boundary $(x=0.6 \mathrm{~m})$. Contaminant species is injected on the inflow boundary located on the left, where a higher pressure condition of $1 \mathrm{~m}-\mathrm{H}_{2} \mathrm{O}$ is imposed. The medium is initially saturate with clean water.

Example 1. Three horizontal and two vertical fractures

The model parameters employed in this base case are listed in table 1 and we attempt to simulate up to 10,000 years. The fracture network involves three horizontal and two vertical fractures that are interconnected (Figure 1). We generate an adaptive triangular mesh for this frac- 
tured media with 43,384 triangles (Figure 2). The triangles are densely and locally refined in the area close to the fractures. As described before, we apply $\mathrm{RT}_{0}-\mathrm{MFE}$ for the flow equation, and semi-implicit FVM-MFE for the transport equation using a uniform time step of 100 years. The simulated pressure field (Figure 3 ) clearly indicates the influence of the fractures on the flow. In particular, two inlets of the fracture network have corresponding local pressure irregularities. The velocity fields are displayed in the streamline/quiver plot (Figure 3). As expected, the magnitude of the velocity is much smaller in the matrix as compared to it in the fracture. Moreover, it can be observed that streamlines tend to converge into fractures in the left part of the domain but diverge from the fractures in the right part, which suggests that factures are the main pathways for transporting contaminants via convection. Figure 4 are results of simulated concentration profiles at different times (100 years, 300 years, 1000 years, 2000 years, 5000 years, and 10,000 years). At 100 years to 1000 years, the contaminant transports mainly through the convection within fractures. This is clearly demonstrated by the concentration plume formed quickly at the fracture outlets while the matrix closer to the inflow boundary is still quite clean. After 2,000 years, diffusion and convection via the matrix also start to play a significant role in the overall contaminant transport behavior.

Example 2. Single fracture

We consider a single fracture in our domain with fracture inlet/outlet not touching the domain boundary. The fracture network is depicted in Figure 5. Even one fracture significantly affects the distribution of pressure and also the streamline (Figure 6). Since streamlines tend to converge into fractures, within about 1,000 years, the concentration plume forms quickly at the outlet. For about ten thousand years, more than $90 \%$ of the region is polluted with the contaminant up to the injected concentration (Figure 7).

Example 3. Two crossed fractures

To compare with the single fracture case, we test another fracture distribution in which there are two crossed fractures in our medium (Figure 8). The other crossed fracture has little influence on the pressure distribution but highly affect the streamline field. More streamlines will be absorbed in this crossed fracture (Figure 9). However, since this fracture blocks pathways of flow around the cross point, the normalized concentration on the right side of the inclined fracture increases slower than it on the left (Figure 10).

Example 4. Single fracture starting at inflow boundary

In the above 3 cases, all fractures does not touch any domain boundary. We now generate a single fracture beginning on the inflow boundary but not reaching on the opposite boundary (Figure 11). The pressure field is significantly impacted by this inlet on the inflow boundary (Figure 12). Unlike the case in Example 2, the fracture attaching on the inflow boundary in this example results into very small pressure drop along the fracture, which explains that the pressure around the fracture is close to the pressure near the inflow boundary. The interaction of this single fracture with the surrounding matrix leads to two low concentration areas downstream to the fracture inlet (Figure 13).

Example 5. Single fracture extending through the domain Similar to the above example, we employ a single fracture, but we now extend it all the way to the outflow boundary (Figure 14). Dramatically different pressure and velocity field are observed from the simulation (Figure 15). One horizontal fracture extending all the way through the entire domain does not have much effect on the pressure pattern (Figure 15). Contaminant 
species passed through the fracture quickly and flow out of our domain in a very short time. This single fracture plays an important role in the transport of chemicals but has less effect on the pressure, velocity and moving direction of normalized concentration in our domain (Figure16).

Example 6. Two crossed fractures with one penetrating the entire domain

When one of the two crossed fractures is penetrating the entire domain (Figure 17), the simulated pressure and velocity (Figure 18) are now completed different from the one we observed in Example 5. Compared with the case in Example 3, the contaminant species convects through fracture much more quickly because the horizontal fracture here connects the inflow and outflow boundaries. As a result, we observe notable difference on the concentration profiles between this example (Figure 19) and Example 3 (Figure 10).

Example 7. Fractures randomly generated in the domain

In Example 7, we randomly generate 3, 6 and 20 fractures in the domain with fracture thickness $0.38 \mathrm{~m}, 0.92 \mathrm{~m}$ and $2.18 \mathrm{~m}$ respectively (Figure 20,23 and 26 ). Since the fracture distribution is more realistic than the other examples above, the streamline and pressure filed are more complicated (Figure 21, 24 and 27). In addition to crucial factors of pressure and streamline, the fracture is also mainly pathways of contaminant species. The normalized concentration in the region enclosed by this fracture network is highly affected by this fracture pattern, since most part of streamlines tends to converge in fractures around. It will take significantly more time to reach the injected concentration level. We present concentration profiles for each example (Figure 22, 25 and 28 ), in which the fracture network highly affect the concentration and streamline distribution during the simulation.

\subsection{Condition numbers of resultant linear systems}

The condition of the algebraic linear systems (9) and (22) is important to our simulation results. At first, we test the condition number of matrix $A_{T}$ in equation (9) with different mesh element quantity and quality (Figure 29). When the mesh is generated uniformly without any fracture in the domain, the condition number of matrix $A_{T}$ maintains a low value as the number of elements is increasing. If some fractures are put into the domain and the mesh is still generated 'uniformly' (the elements near or in the fracture will not be uniformly generated), the condition number of matrix $A_{T}$ is significantly increased to infinity which means it is nearly singular. Thus we need to use adaptive mesh to complete our simulation. The condition numbers of matrix $A_{T}$ with different number of adaptive mesh elements are shown in (Figure 29). We can see that the condition of the matrix is slightly worse than the matrix of the uniform mesh without fractures, but much better than that of the uniform mesh with the same number of fractures. The relation between the fracture density and the condition number of matrix $A_{T}$ is also investigated in this paper (Figure 30). The condition of the matrix will become worse when the fracture density is increasing significantly.

Then we consider the equation (22). When the whole system reaches its equilibrium, we only need to take care of the condition of matrix $A=\tilde{A}_{T}+\hat{A}_{T}$. Figure 31 represents the condition numbers of this matrix as the mesh quality and the number of elements change. The same result as that in the equation (9) is concluded. The adaptive mesh significantly contributes to the 
condition of matrix $A$ and enhances the accuracy of our simulation results. Also as the number of elements increases, the condition of matrix $A$ increases as expected (Figure 32).

\subsection{Sensitivity Analysis Effect of fracture thickness}

To analyze the effect of fracture pattern, we test three cases in Example 7. We use average effluent concentration to test the influence of fracture thickness. The average effluent concentration $\bar{c}$ is defined as follows:

$$
\bar{c}=\frac{\int_{\Gamma_{\text {ouffow }}} \mathrm{u} \cdot n c d s}{\int_{\Gamma_{\text {ouffow }}} \mathrm{u} \cdot n d s}
$$

Different fracture thickness is employed and the average effluent concentration on outflow boundary versus time is plotted in Figure 33. The average effluent concentration on the outflow boundary for the case with higher fracture thickness increases more quickly during our simulation time than those with lower fracture thickness.

\section{Effect of diffusion coefficient}

We use 20 randomly generated fractures media in Example 7 to analyze the effect of diffusivity. The saturated effective diffusion coefficient is changed from the low value $5 \times 10^{-12} \mathrm{~m}^{2} / \mathrm{s}$ to the high value $5 \times 10^{-5} \mathrm{~m}^{2} / \mathrm{s}$ (Figure 34). It spends more time to reach $50 \%$ concentration with higher diffusivity, however, it takes less time to reach a higher value like $90 \%$ concentration.

\section{Effect of $K_{d}$ value}

$\mathrm{K}_{\mathrm{d}}$, which represent the distribution of contaminant between the solid and liquid phases, is defined as follows (Freeze and Cherry, 1979 and Burkholder, 1976),

$$
K_{d}=\frac{\text { mass of solute on the solid phase per unit mass of solid phase }}{\text { concentration of solute in solution }}
$$

The effect of the $K_{d}$ expresses as a retardation in the breakthrough curve of a contaminant. The retardation of the contaminant front relative to the bulk water is

$$
\frac{\bar{v}}{\bar{v}_{c}}=1+\frac{\rho_{b}}{n} \bullet K_{d}
$$

where $\bar{v}$ is the average linear velocity of the groundwater, $\bar{v}_{c}$ is the velocity of the $\mathrm{C} / \mathrm{C}_{0}=0.5$ point of the concentration profile of the retarded constituent, $\rho_{b}$ is the bulk porous medium density, and $n$ is the porosity of the porous medium.

We use the 20 randomly generated fractures media in Example 7 to test the effect of $K_{d}$ value. The $K_{d}$ value is changed from standard value $0 \mathrm{ml} / \mathrm{g}$ (no adsorption) to high value $0.5 \mathrm{ml} / \mathrm{g}$ (high adsorption), with the results displayed in Figure 35. It takes more time for the normalized concentration on the outflow boundary to reach any fixed concentration value with higher $\mathrm{K}_{\mathrm{d}}$ value. 
4.3 Lumping an entire fracture network into one equivalent crack via effective $K_{d}$ Although the retardation equation for fractured media is quite simple, its application is quite difficult due to the issue of actually knowing how the fractures appear. This paper addresses the issue of how to apply this equation by numerically solving the transport equation for various combinations of fractures and then developing a relationship between intrinsic $K_{d}$ value and effective $K_{d}$ value.

In this section, we calculate the effective $K_{d}$ value by lumping a complicated fractured network into a simple network. We use average effluent concentration on the outflow boundary to build a relation between intrinsic $K_{d}$ value of fracture network (Figure 26) and effective $K_{d}$ value of fracture network (Figure 5). At first, we use our flow and contaminant transport simulator to obtain the relationship between $50 \%$ average effluent concentration time and intrinsic $K_{d}$ values (Figure 36). Next, we calculate the relationship between $50 \%$ average effluent concentration time and effective $K_{d}$ values (Figure 36) for a single-fracture network. We find that both of these two relations appear to be linear in the range of $K_{d}$ value from $0 \mathrm{ml} / \mathrm{g}$ to $5 \mathrm{ml} / \mathrm{g}$. Then, from these two linear relations, we can conclude that the relation between intrinsic $K_{d}$ value and effective $K_{d}$ value is also linear. To verify this conclusion, we test relationship between effective $K_{d}$ and intrinsic $K_{d}$ over the same $50 \%$ average effluent concentration time. The final result we obtained is depicted in Figure 37. The relationship between the effective $K_{d}$ is the intrinsic $K_{d}$ appears to be strictly linear from 0 to 5 . This investigation indicates that effective $K_{d}$ can be computed from intrinsic $K_{d}$ by applying a multiplier.

\section{Conclusions and future work}

In this paper, an efficient and robust simulator has been developed for the solution of contaminant species passing through a fractured cementitious matrix. We first presented our mathematical model consisting of two differential equations, i.e. the flow equation and the reactive transport equation. A numerical scheme based on the mixed finite element (MFE) method is developed to approximate the second-order partial derivate terms in the flow and transport equations. The convection term in the transport equation is treated using an upwind finite volume method (FVM). With the MFE method, the fluxes through fractures are accurately approximated using the adaptive conforming triangular mesh since the triangular grids have the potential for effective mesh adaptation. Various patterns of fractures are simulated and compared. In the triangular computation, we locally refine the elements in the areas close to fractures for improved accuracy and efficiency of the numerical solution. Sensitivity analysis has been carried out for the simulations by perturbing several parameters. The effective $K_{d}$ calculation for lumping an entire crack network to an equivalent single-fracture system has been proposed and carried out using our contaminant transport simulator. In a near future, we will investigate the relationship between lumped effective $K_{d}$ and fracture density, which we believe is meaningful and useful and plays a crucial rule in the contaminant transport system and it tightly interacts with many other parameters in our mathematics model such as conductivity, porosity and intrinsic $K_{d}$, thus affecting the lumped effective $K_{d}$. Moreover, we also plan to study more effective mesh adaptation for our triangular discretization, especially the adaptivity guided by physical quantities important to our system such as flow rate and concentration gradients.

Acknowledgements: This work has been supported by a research grant through the Savannah River National Laboratory (SRNL). The authors would also like to thank Dr. Richard A. Dimenna in SRNL for his insightful discussion and constructive suggestions. 


\section{Reference}

Arbogast, T., J. Douglas, and U. Hornung (1990), Derivation of the double porosity model of single phase via homogenization theory, SIAM J. Math. Anal., 21: 823-836.

Baca, R., R. Arnett, and D. Langford (1984), Modeling fluid flow in fractured porous rock masses by finite element techniques, Int. J. Numer. Methods Fluids, 4: 337- 348.

Bastian, P., R. Helmig, H. Jakobs, and V. Reichenberger (2000), Numerical simulation of multiphase flow in fractured porous media, in Numerical Treatment of Multiphase Flows in Porous Media, Lect. Notes Phys., vol. 552, pp. 1- 18, Springer, New York.

Bear, J., C.F. Tsang, and G. de Marsily (ed.) (1993). "Flow and contaminant transport in fractured rock," San Diego: Academic Press.

Bodvarsson, G.S., Y.S. Wu, and K. Zhang. (2002). Development of discrete flow paths in unsaturated fractures. J. Contam. Hydrol. 62-63:23-42.

Brezzi, F., \& Fortin, M. (1991), "Mixed and hybrid finite element methods," New York: SpringerVerlag.

Burkholder, H. C. (1976), Methods and data for predicting nuclide migration in geologic media. International Symposium on Management of Wastes from the LWR Fuel Cycle, Denver, Colorado.

Chavent, G., and J.-E. Roberts (1991), A unified physical presentation of mixed, mixed-hybrid finite element method and standard finite difference approximations for the determination of velocities in water flow problems, Adv. Water Resour., 14(6): 329-348.

Ewing, R. E., Lazarov, R. D., \& Wang, J. (1991), Superconvergence of the velocity along the gauss lines in mixed finite element methods. SIAM J. Numer. Anal., 28: 1015-1029.

Freeze, R. A. and Cherry, J. A. (1979), “Groundwater," New Jersey: Prentice-Hall, Inc.

Glass, R.J., M.J. Nicholl, and V.C. Tidwell (1996), Challenging and improving conceptual models for isothermal flow in unsaturated, fractured rocks through exploration of small-scale processes. Rep. SAND95-1824. Sandia National Laboratories, Albuquerque, NM.

Granet, S., P. Fabrie, P. Lemmonier, and M. Quitard (1998), A single phase flow simulation of fractured reservoir using a discrete representation of fractures, in paper presented at 6th European Conference on the Mathematics of Oil Recovery (ECMOR VI), Eur. Assoc. of Geosci. and Eng., Peebles, U. K.

H. Hoteit and A. Firoozabadi(2005), Multicomponent fluid flow by discontinuous Galerkin and mixed methods in unfractured and fractured media, Water Resour. Res., 41: W11412.

Hoa Nguyen, John Burkardt, Max Gunzburger, Lili Ju, Yuki Saka (2009), Constrained CVT Meshes and a Comparison of Triangular Mesh Generators, Computational Geometry: Theory and Applications, Volume 42, Number 1: 1-19. 
J. Shewchuk (1996), Triangle: Engineering a 2D quality mesh generator and Delaunay triangulator, in: Lecture Notes in Comput. Sci., vol. 1148, Springer, New York, 203-222.

Karimi-Fard M., and A. Firoozabadi (2003), Numerical simulation of water injection in 2-D fractured media using discrete-fracture model, SPE Revervoir Eval. Eng., 4: 117-126.

Kazemi, H. (1969), Pressure transient analysis of naturally fractured reservoirs with uniform fracture distribution, SPE J., 9: 451-462.

Kim, J., and M. Deo (1999), Comparison of the performance of a discrete fracture multiphase model with those using conventional methods, paper SPE 51928 presented at SPE Reservoir Simulation Symposium, Soc. Of Pet. Eng., Houston, Tex.

Kim, J., and M. Deo (2000), Finite element, discrete fracture model for multiphase flow in porous media, AIChE J., 46(6): 1120-1130.

Liu, H.H., and G.S. Bodvarsson (2001), Constitutive relation for unsaturated flow in fractured networks, J.Hydrol. (Amsterdam) 252:116-125.

L. Ju (2007), Conforming centroidal Voronoi Delaunay triangulation for quality mesh generation, Inter. J. Numer. Anal. Model. 4: 531-547.

National Research Council. (1996). Rock fractures and fluid flow: Con temporary understanding and applications. National Academy Press, Washington, DC.

Noorishad, J., and M. Mehran (1982), An upstream finite element method for solution of transient transport equation in fractured porous media, Water Resour. Res., 18(3), 588-596.

Pruess, K., B. Faybishenko, and G.S. Bodvarsson. (1999). Alternative concepts and approaches for modeling flow and transport in thick unsaturated zones of fractured rocks. J. Contam. Hydrol. 38:281-322.

P. -O. Persson, G. Strang. (2004). A Simple Mesh Generator in MATLAB. SIAM Review, Volume 46 (2), 329-345.

Raviart, R.A and Thomas, J.M(1977). A mixed finite method for $2^{\text {nd }}$ order elliptic problems, in Mathematical Aspects of the Finite Element Method, Lecture Notes in March. 606, SpringerVerlag, New York,292-315.

Su, G.W., J.T. Geller, K. Pruess, and F. Wen. (1999). Experimental studies of water seepage and intermittent flow in unsaturated rough-walled fractures. Water Resour. Res. 35:1019-1037.

Thomas, L., T. Dixon, and R. Pierson (1983), Fractured reservoir simulation,SPE J., 23: 42- 54.

Tokunaga, T.K., and J. Wan. (1997). Water film flow along fracture surface of porous rock. Water Resour. Res. 33:1287-1295.

Warren, J., and P. Root (1963), The behavior of naturally fractured reservoirs, SPE J., 3: 245- 255. 
Table 1. Standard parameters

\begin{tabular}{cccc}
\hline $\begin{array}{c}\text { Saturated Hydraulic } \\
\text { Conductivity, Ks } \\
(\mathrm{cm} / \mathrm{s})\end{array}$ & $\begin{array}{c}\text { Saturated Effective } \\
\text { Diffusion Coefficient, } \\
\mathrm{D}_{\mathrm{e}}(\mathrm{cm} 2 / \mathrm{s})\end{array}$ & $\begin{array}{c}\text { Effective } \\
\text { Porosity } \\
(\%)\end{array}$ & $\mathrm{K}_{\mathrm{d}}$ value \\
\hline $1.0 \mathrm{E}-12$ & $5.0 \mathrm{E}-11$ & 18.4 & 0 \\
\hline
\end{tabular}

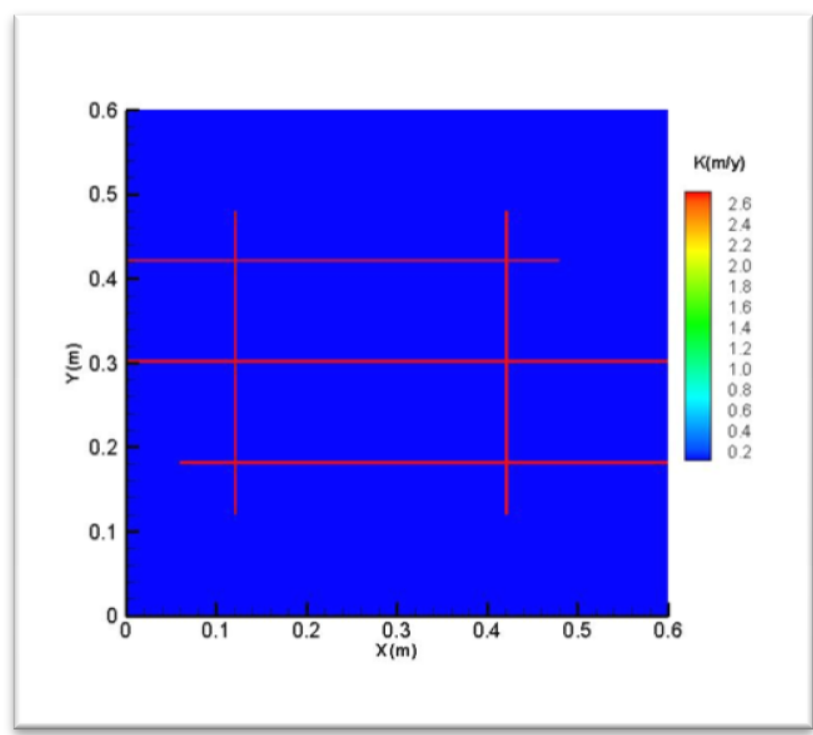

Figure 1. Enlarged fracture and conductivity distribution for base case 


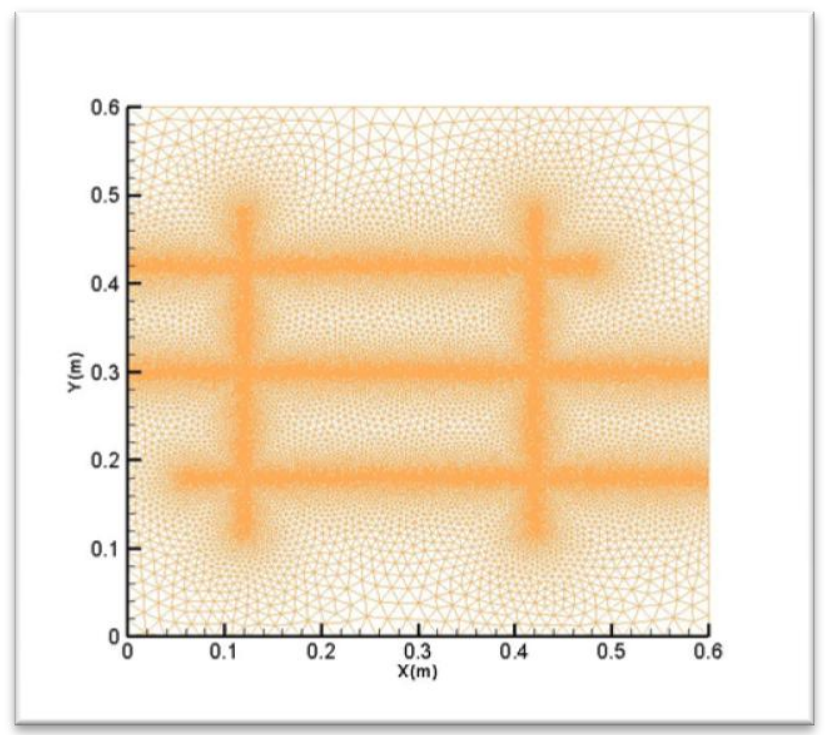

Figure 2. Adaptive triangular mesh for base case

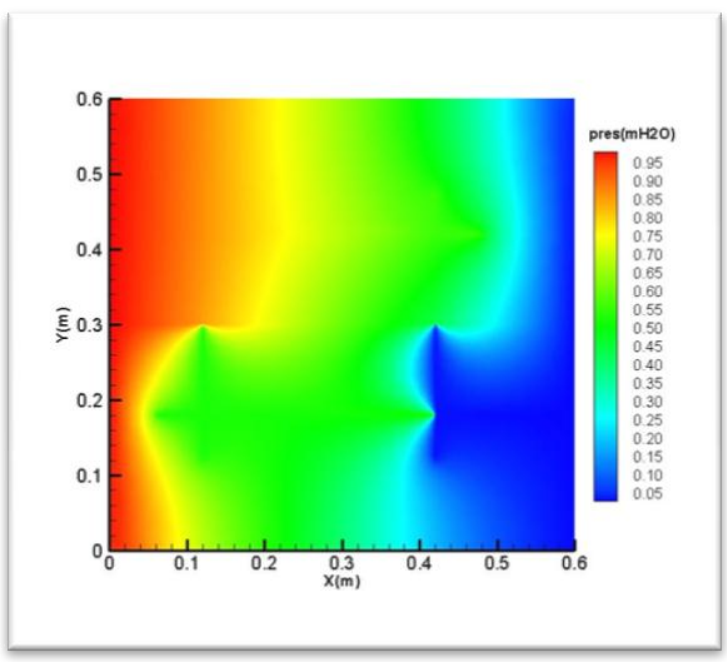

Pressure distribution

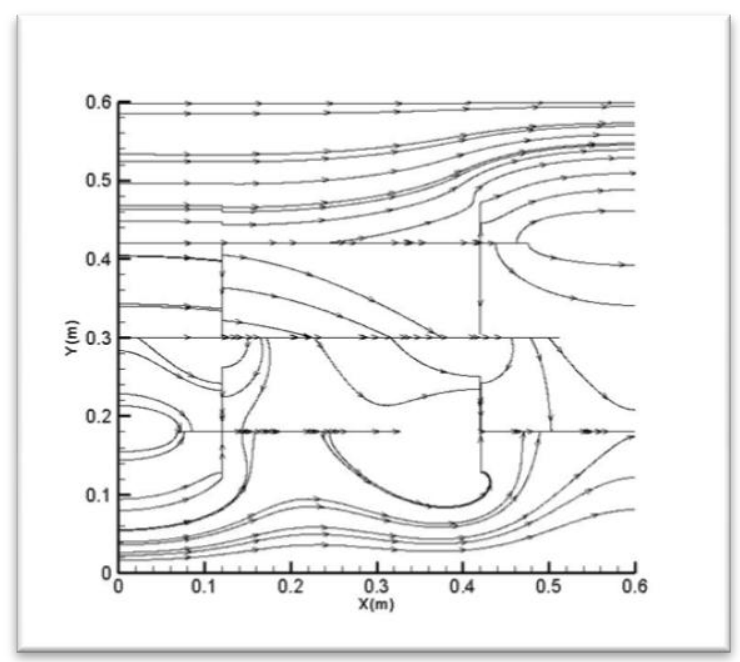

Streamline field

Figure 3. Pressure distribution and Streamline filed for Example 1 


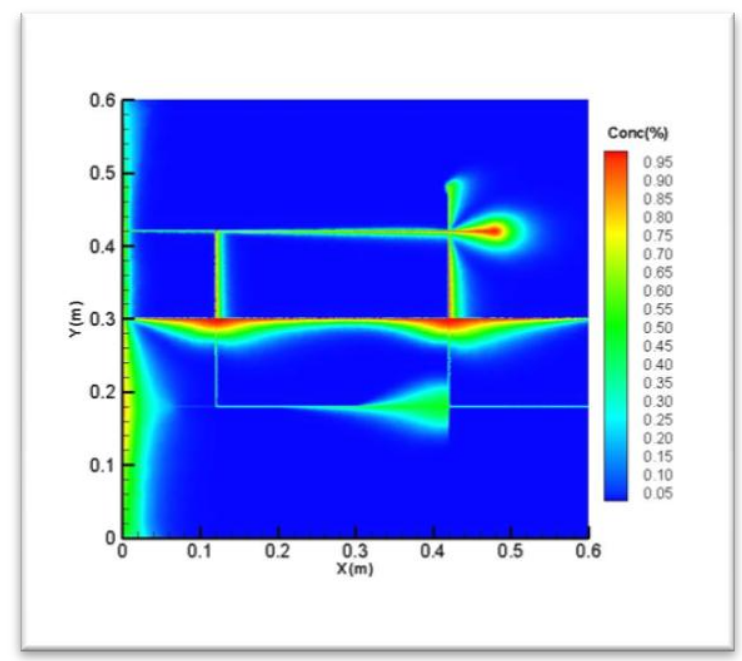

Concentration distribution at 100year

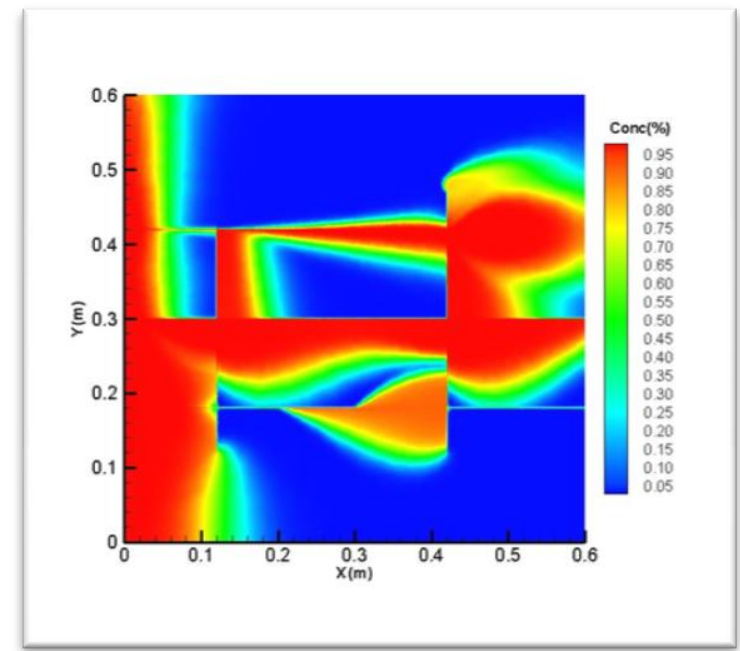

Concentration distribution at 1000 year

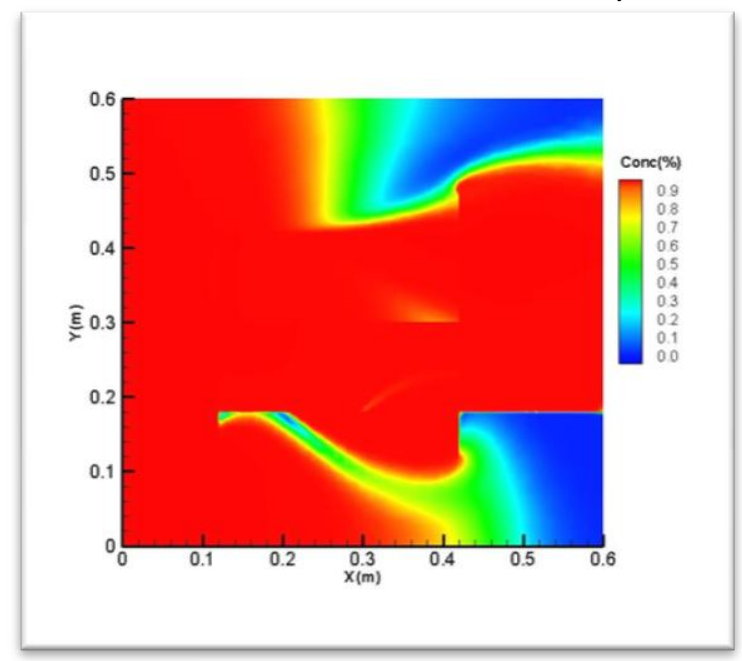

Concentration distribution at 5000 year

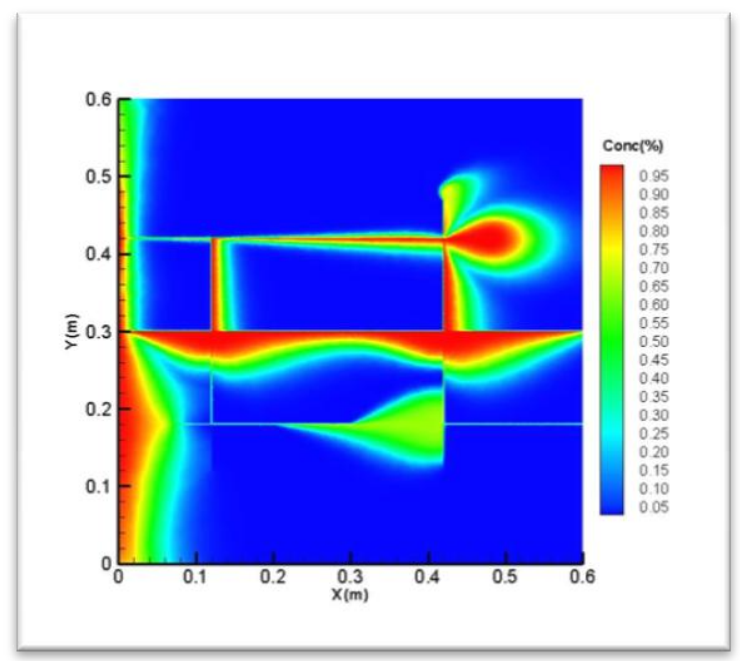

Concentration distribution at 300 year

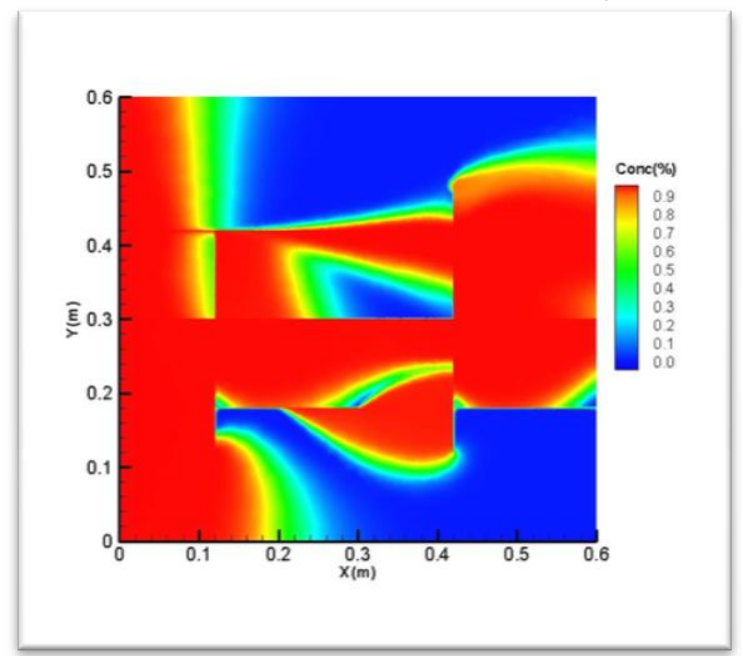

Concentration distribution at 2000 year

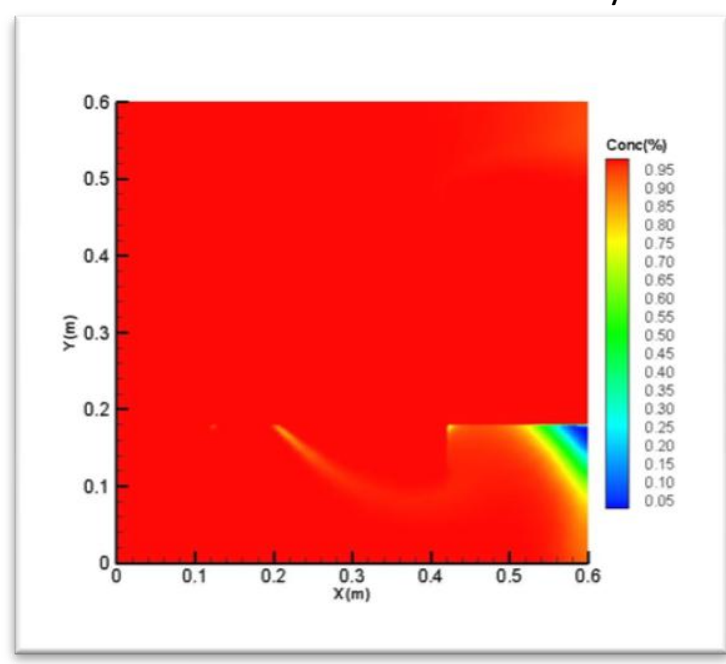

Concentration distribution at 10000 year Figure 4. Concentration at different time within ten thousand years 


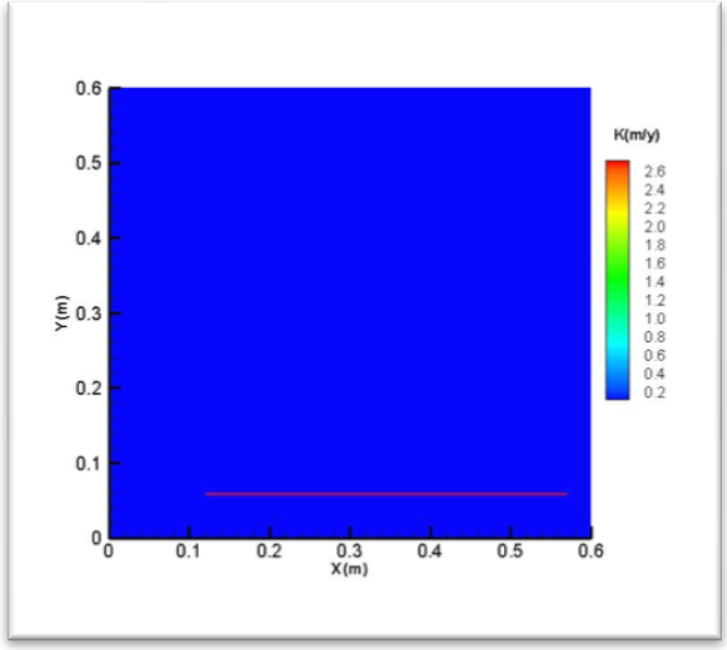

Enlarged fracture and conductivity distribution

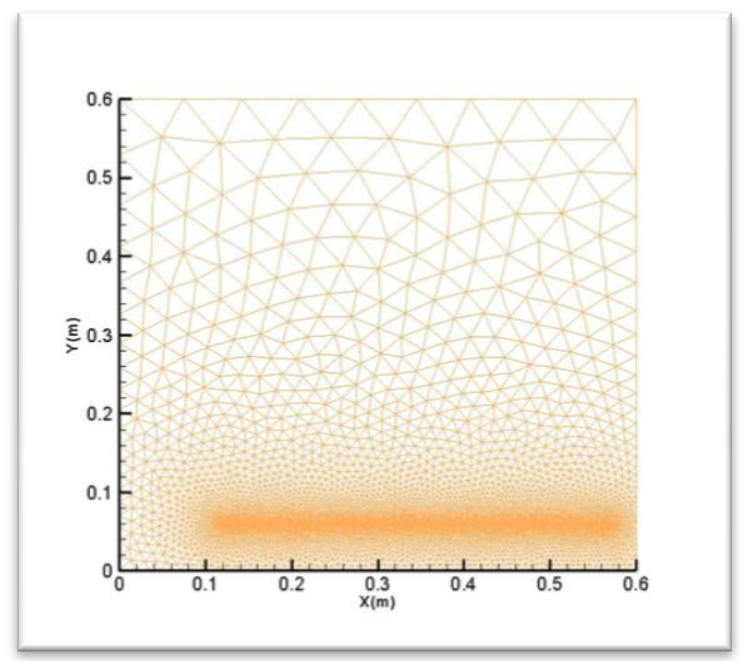

Triangular mesh for single fracture

Figure 5. Fracture network for single fracture with no begin at any boundary

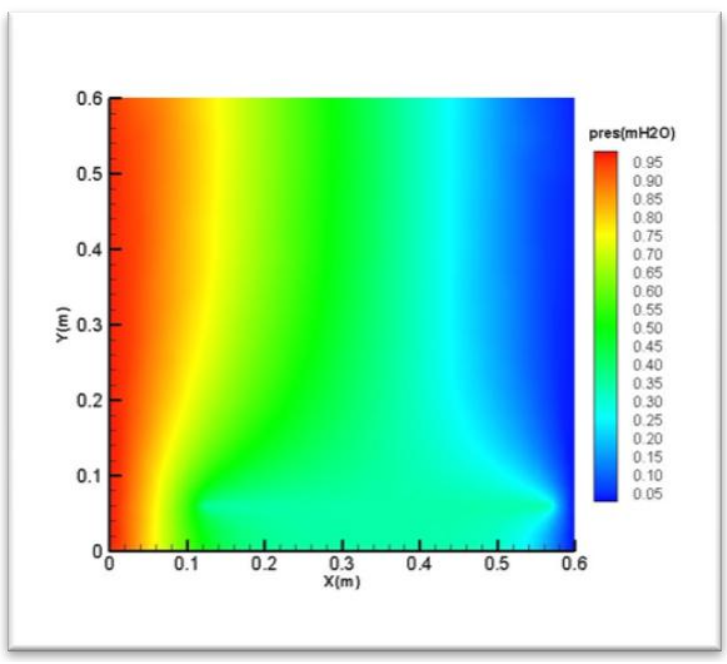

Pressure distribution

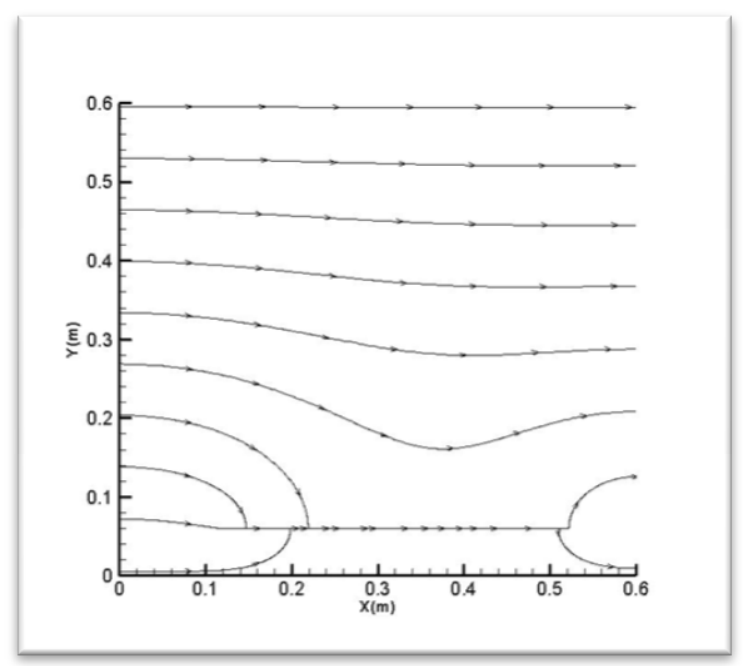

Streamline field

Figure 6. Pressure distribution and Streamline field for single fracture 


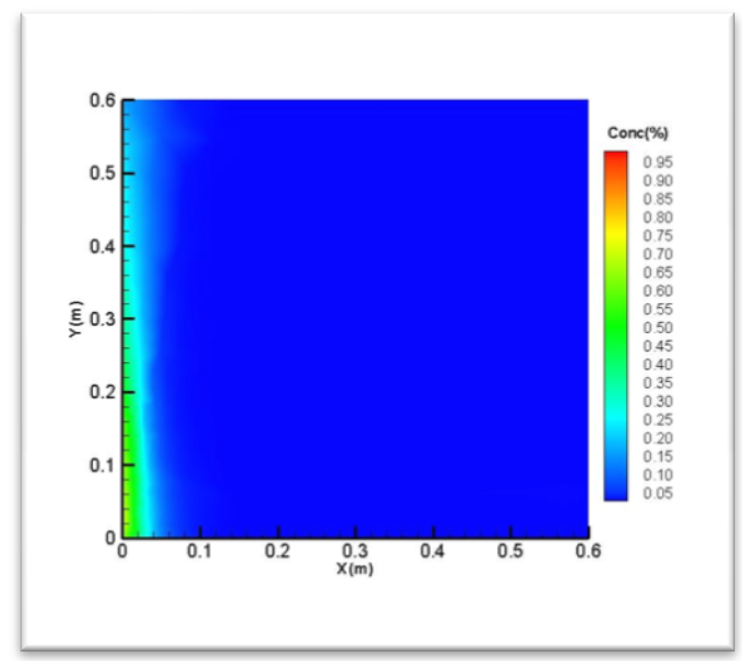

Concentration distribution at 100 year

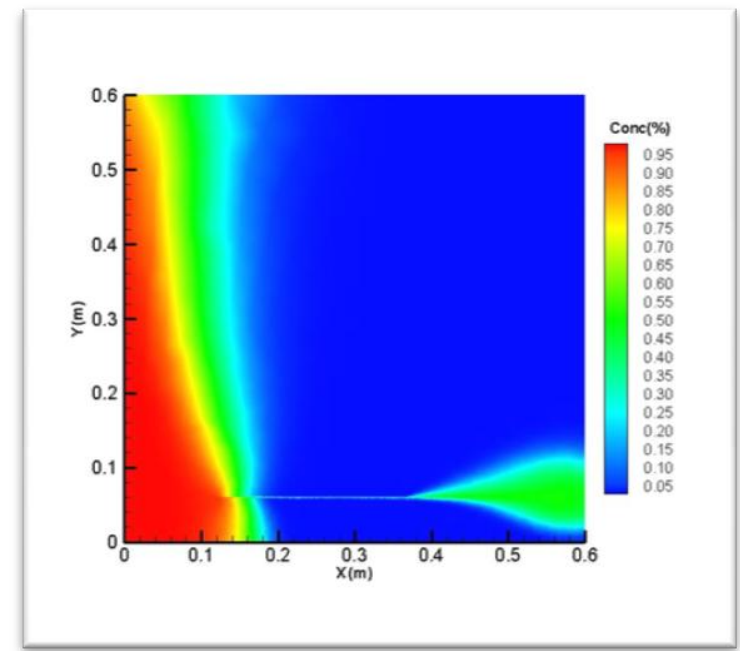

Concentration distribution at 1000 year

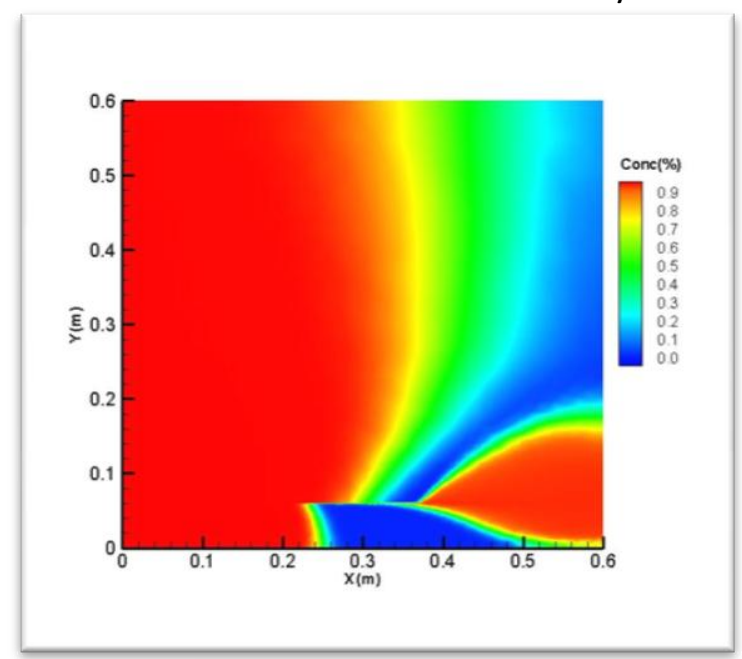

Concentration distribution at 5000 year

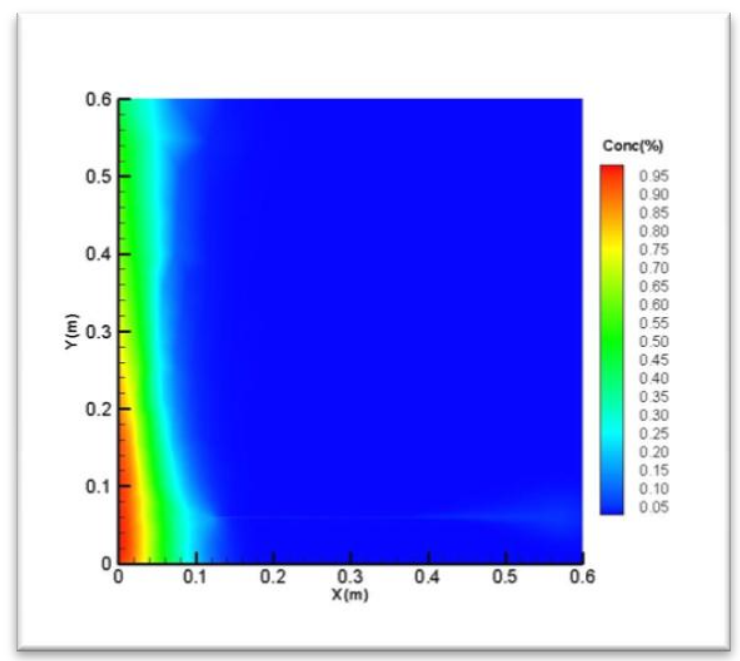

Concentration distribution at 300 year

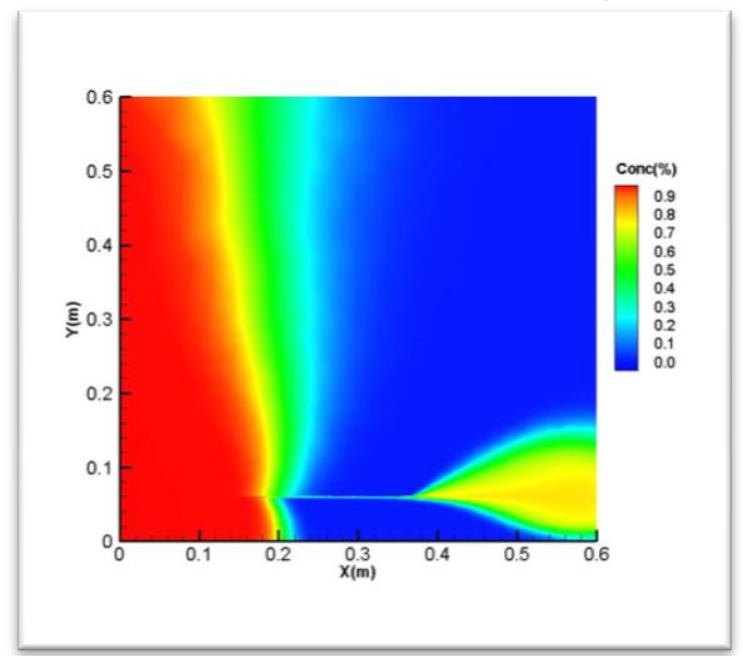

Concentration distribution at 2000 year

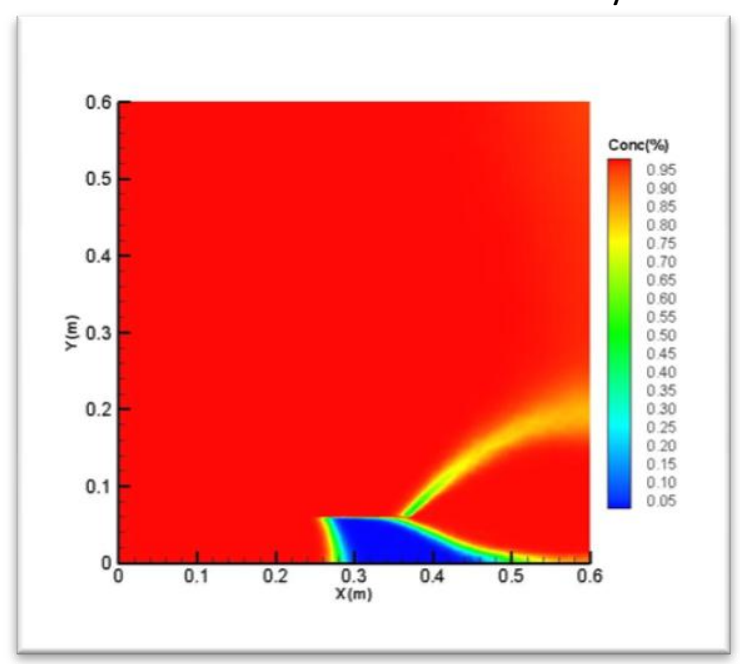

Concentration distribution at 10,000 year Figure 7. Concentration at different time within ten thousand years of single fracture 


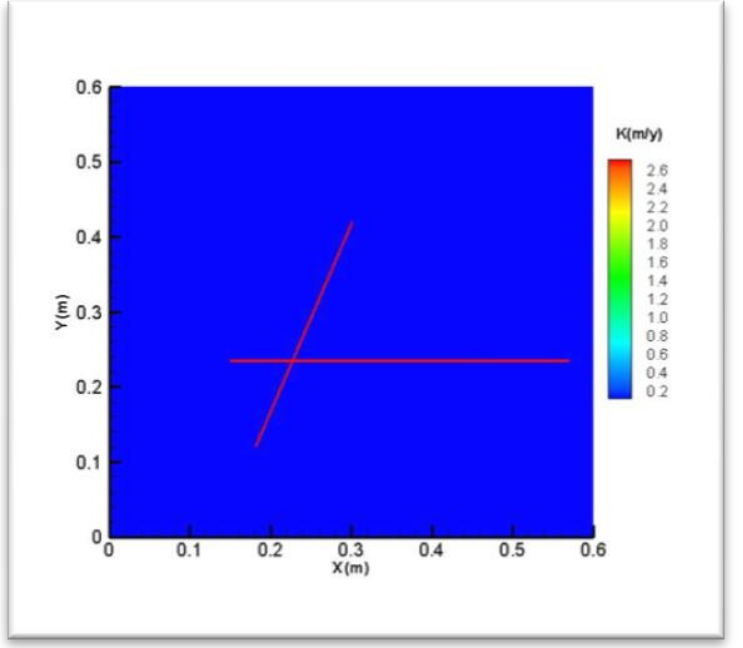

Enlarged fracture and conductivity distribution

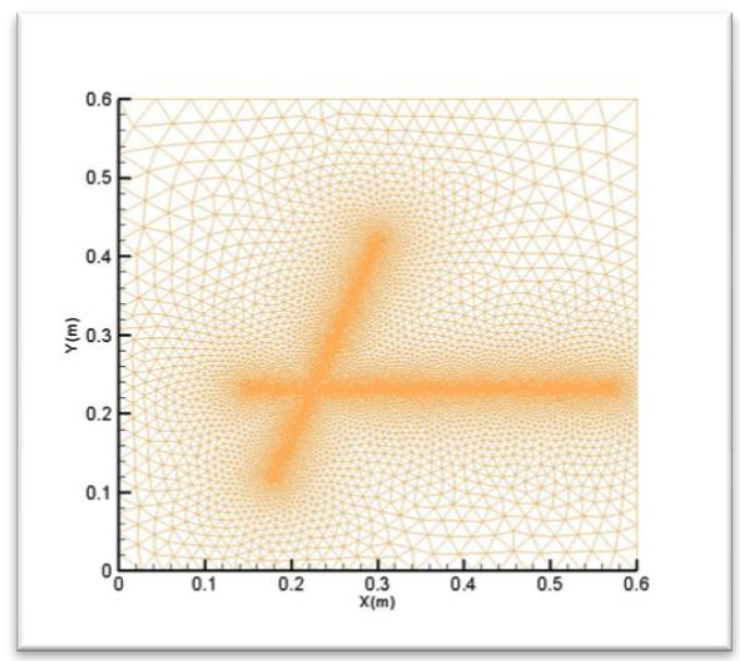

Triangular mesh for single fracture

Figure 8 . Fracture network for two crossed fracture with no begin at any boundary

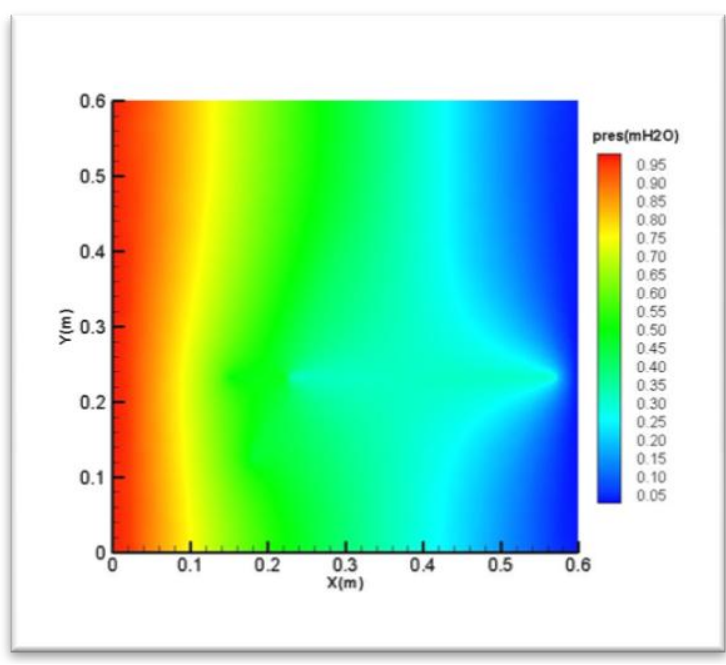

Pressure distribution

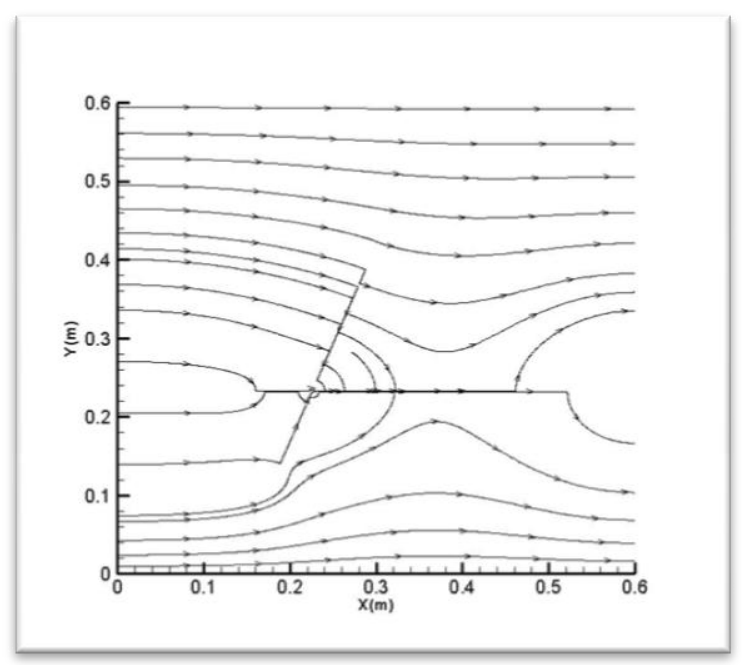

Streamline field

Figure 9. Pressure distribution and Streamline filed for two crossed fractures 


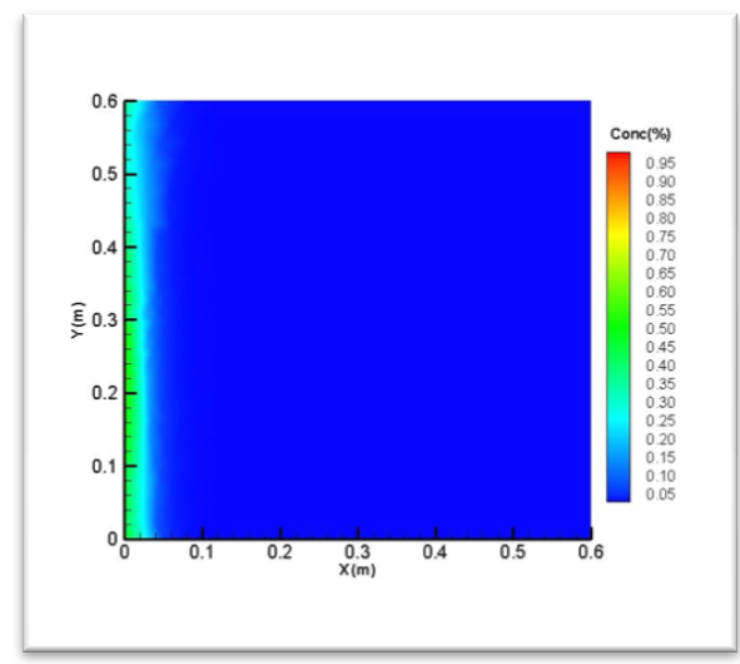

Concentration distribution at 100 year

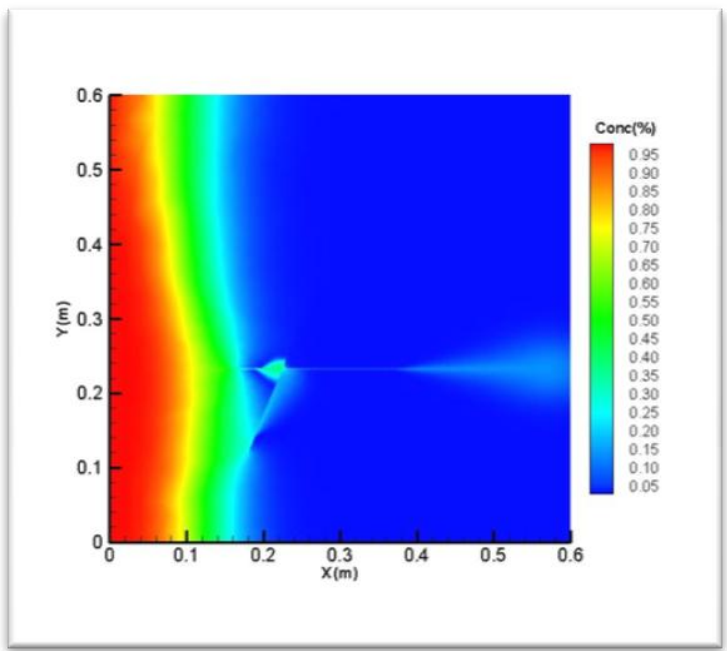

Concentration distribution at 1000 year

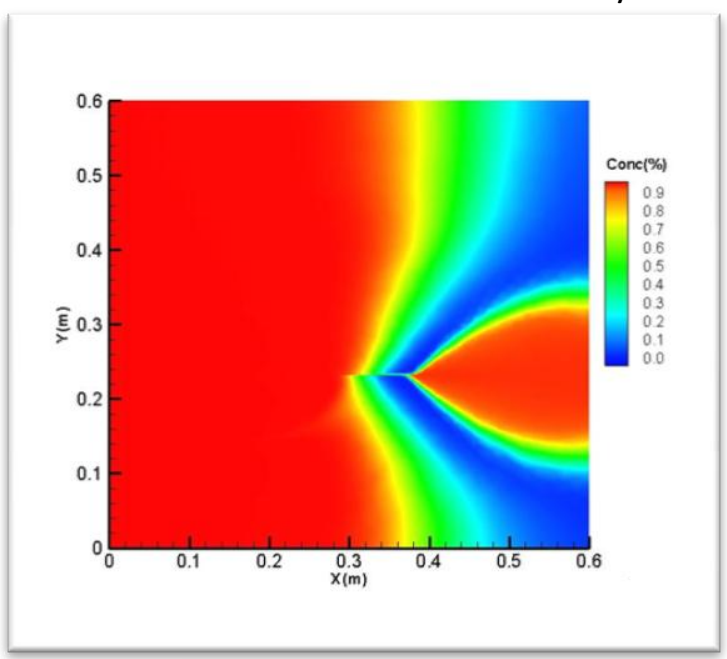

Concentration distribution at 5000 year

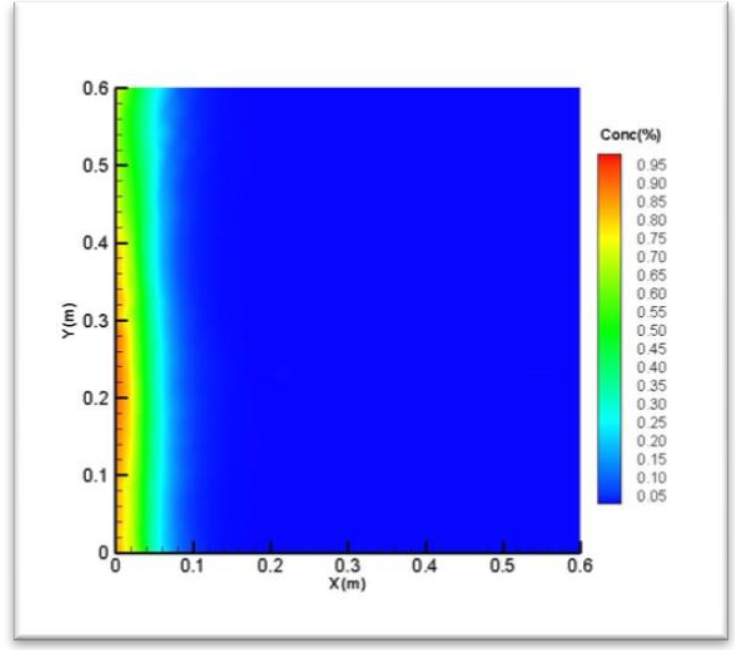

Concentration distribution at 300 year

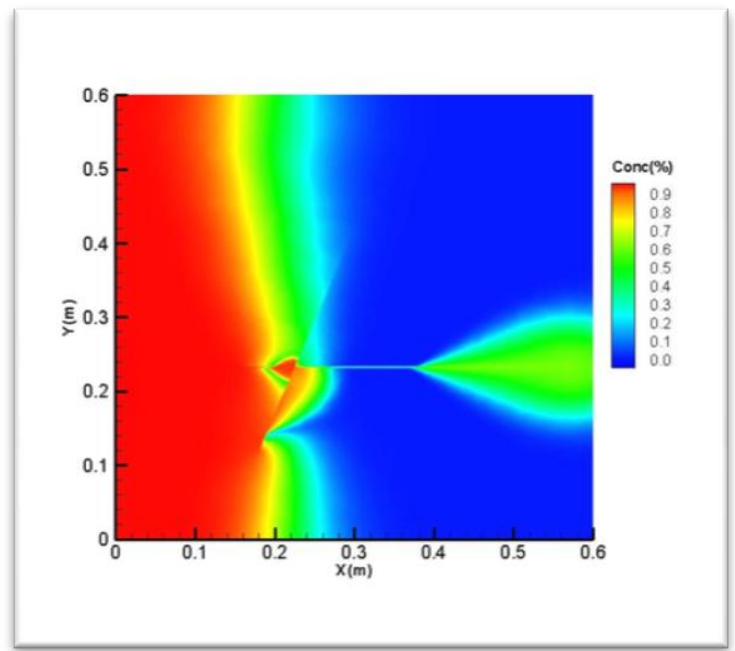

Concentration distribution at 2000 year

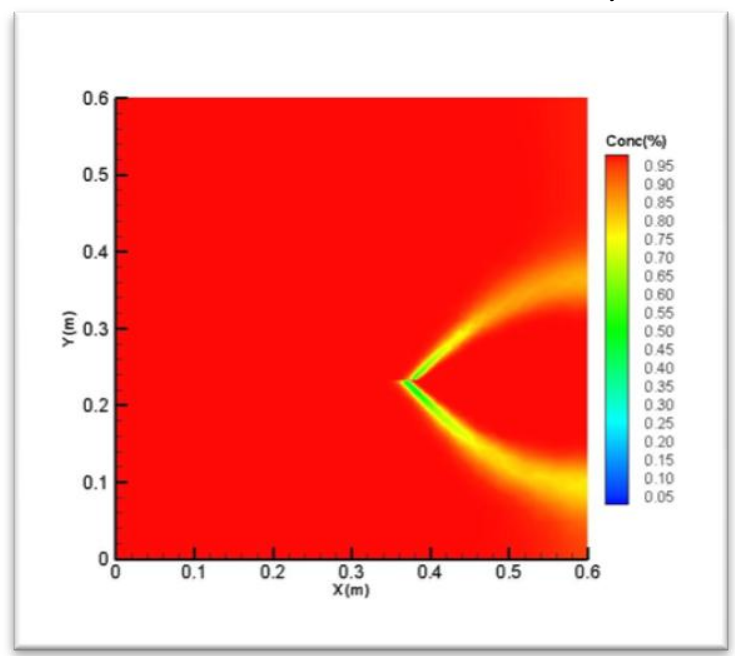

Concentration distribution at 10000 year Figure 10. Concentration at different time within ten thousand years of two crossed fracture 


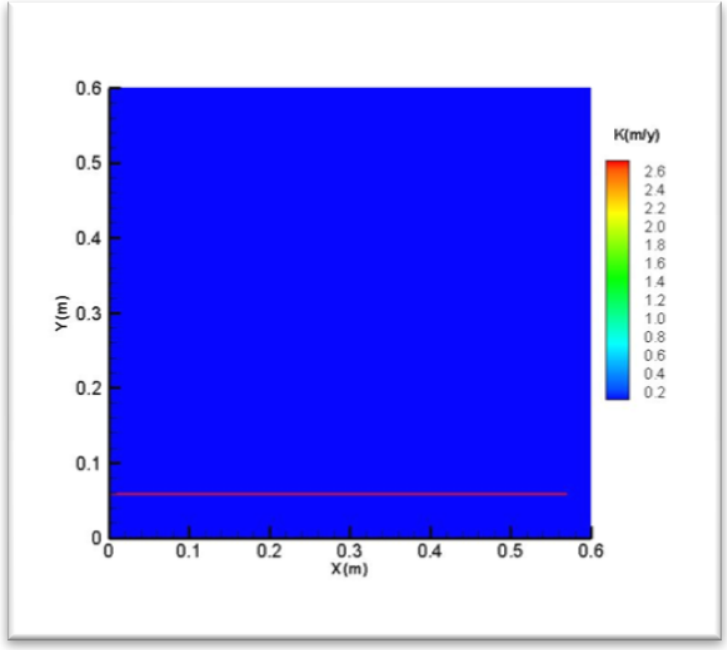

Enlarged fracture and conductivity distribution

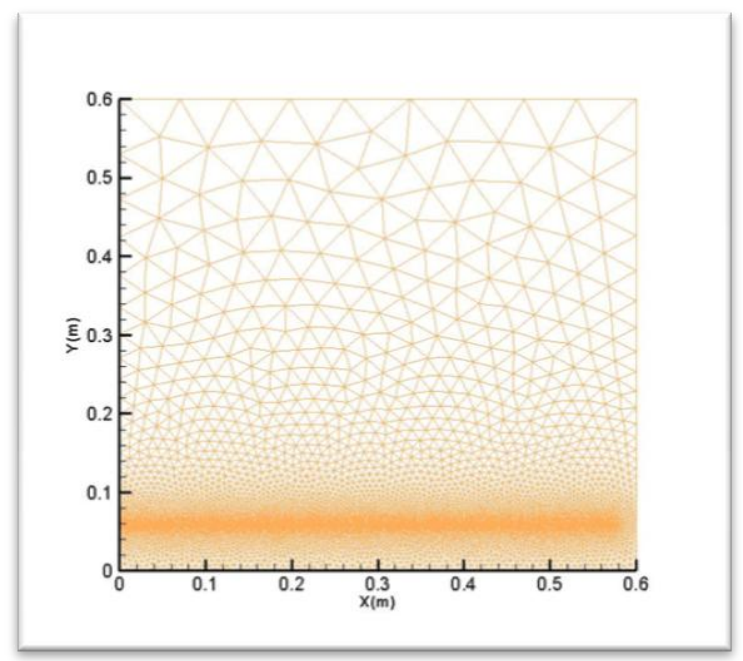

Triangular mesh for single fracture

Figure 11. Fracture network for single fracture cross inflow boundary

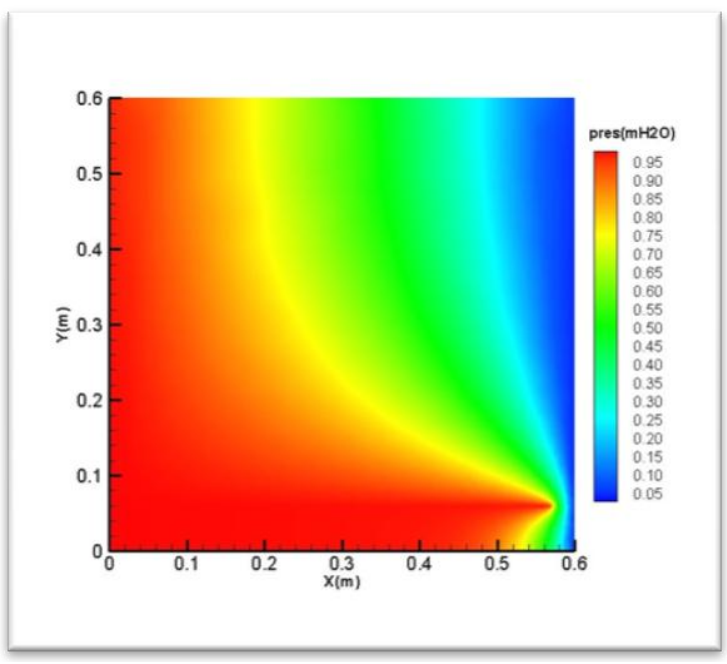

Pressure distribution

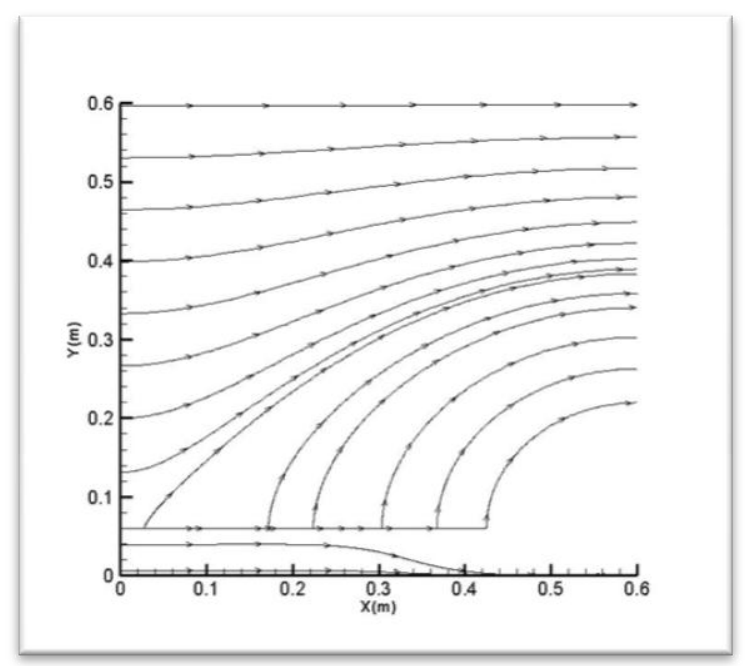

Streamline field

Figure 12. Pressure distribution and Streamline filed for one fracture cross inflow boundary 


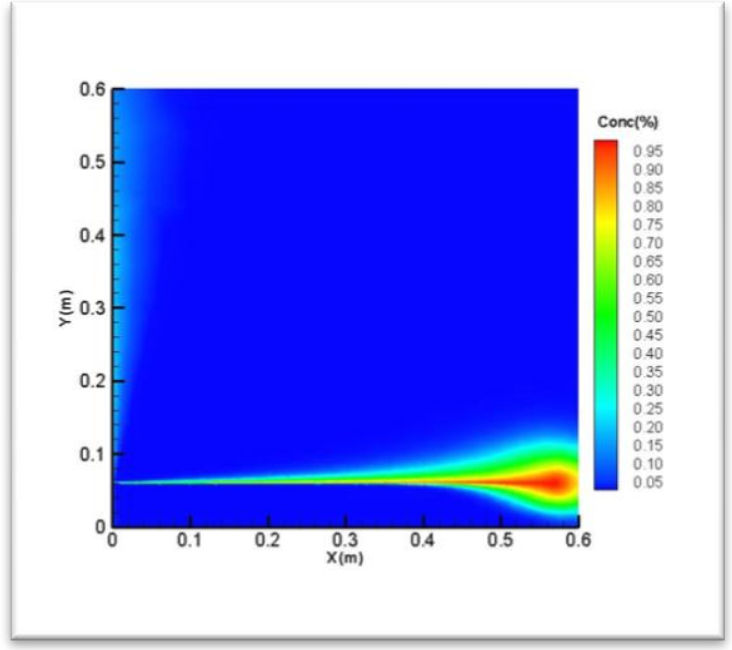

Concentration distribution at 100 year

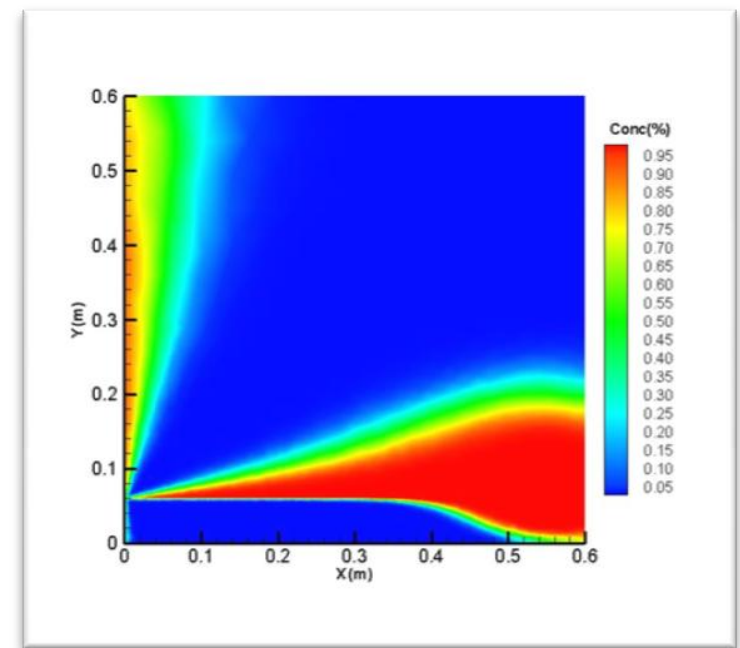

Concentration distribution at 1000 year

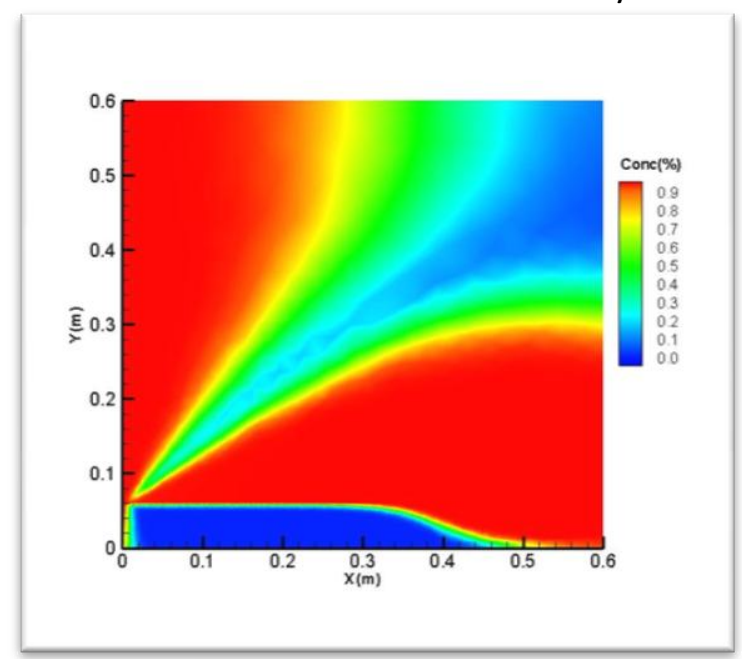

Concentration distribution at 5000 year

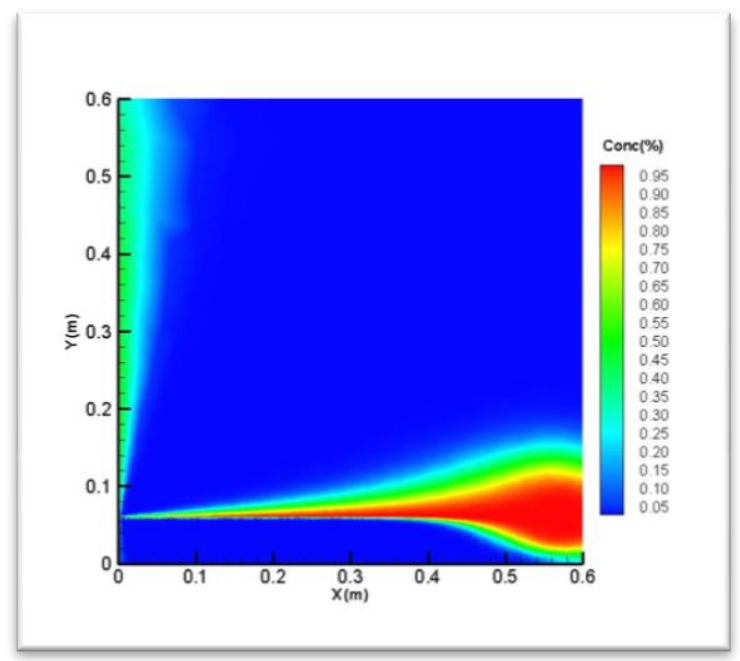

Concentration distribution at 300 year

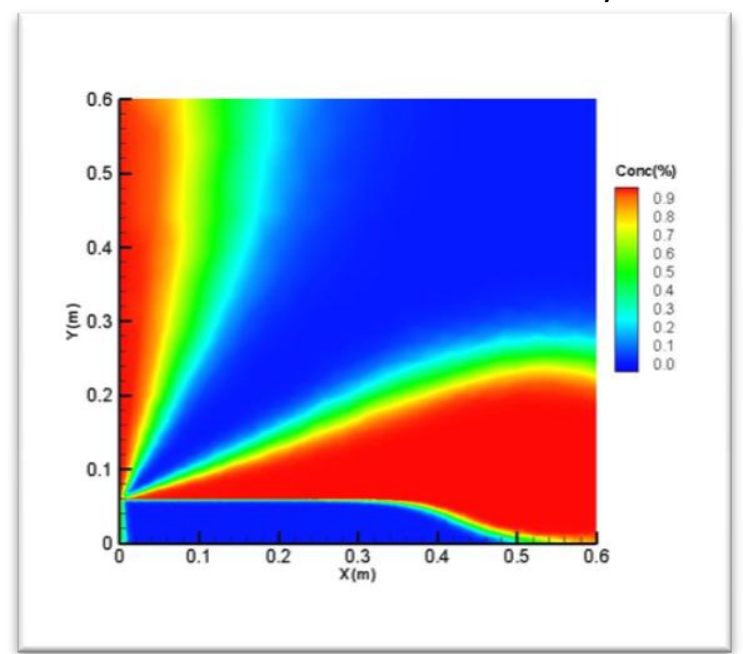

Concentration distribution at 2000 year

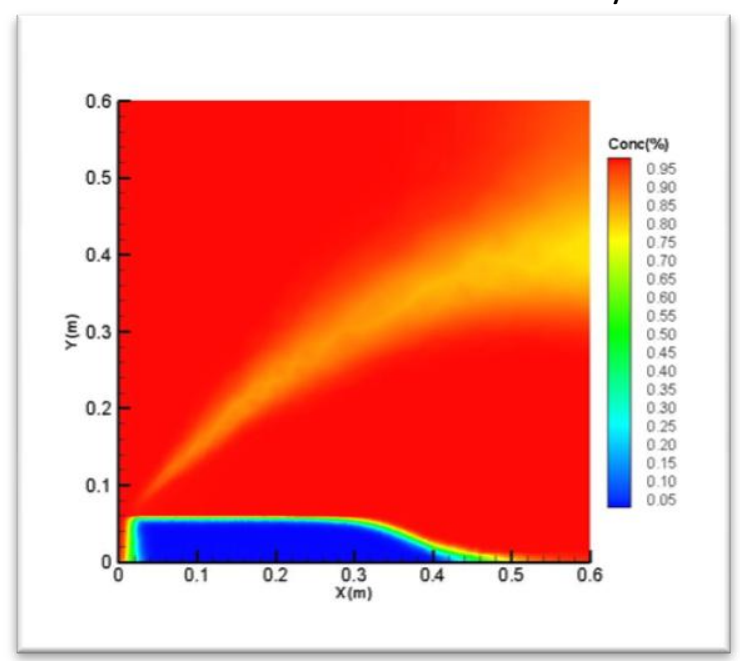

Concentration distribution at 10000 year

Figure 13. Concentration at different time within ten thousand years for one fracture cross inflow boundaries 


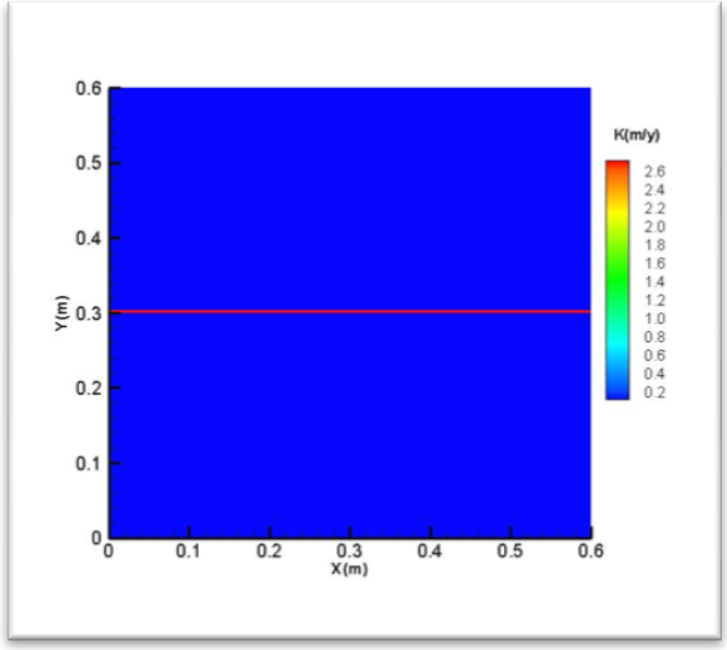

Enlarged fracture and conductivity distribution

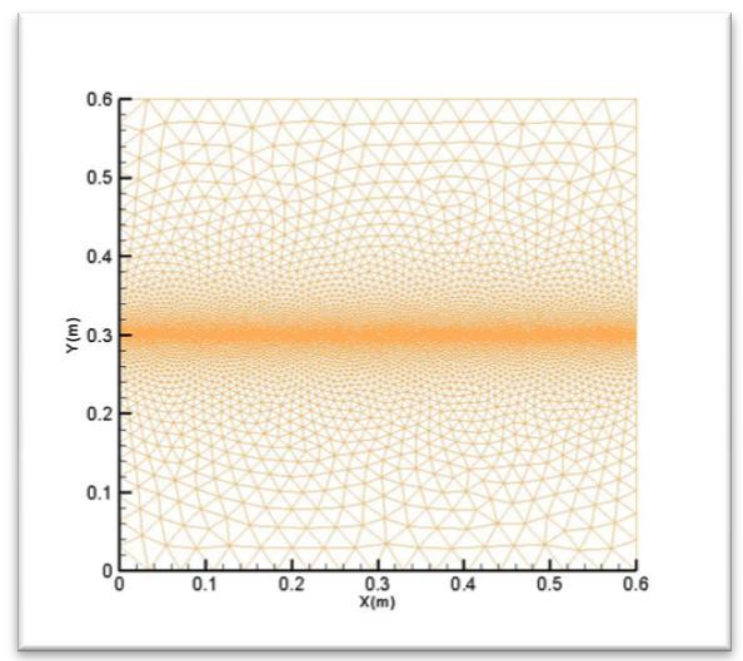

Triangular mesh for single fracture

Figure 14. Fracture network for single fracture cross two boundaries

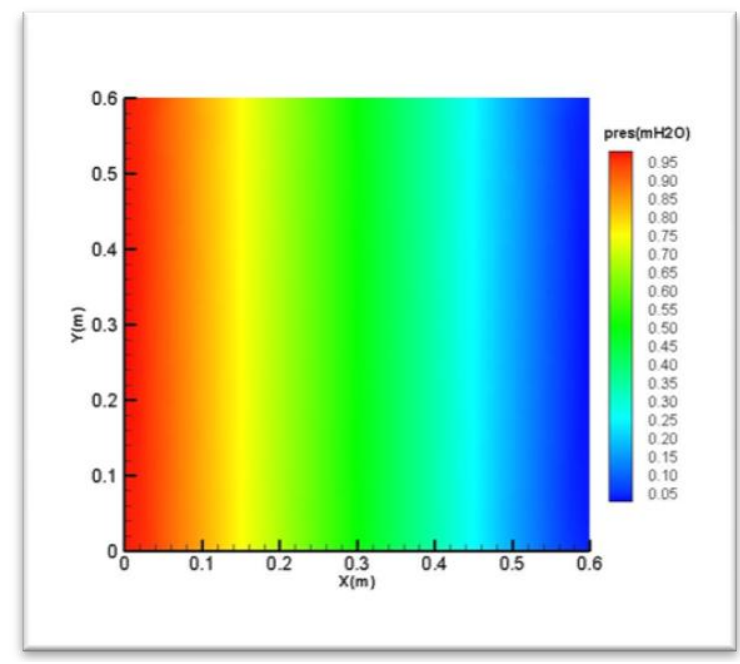

Pressure distribution

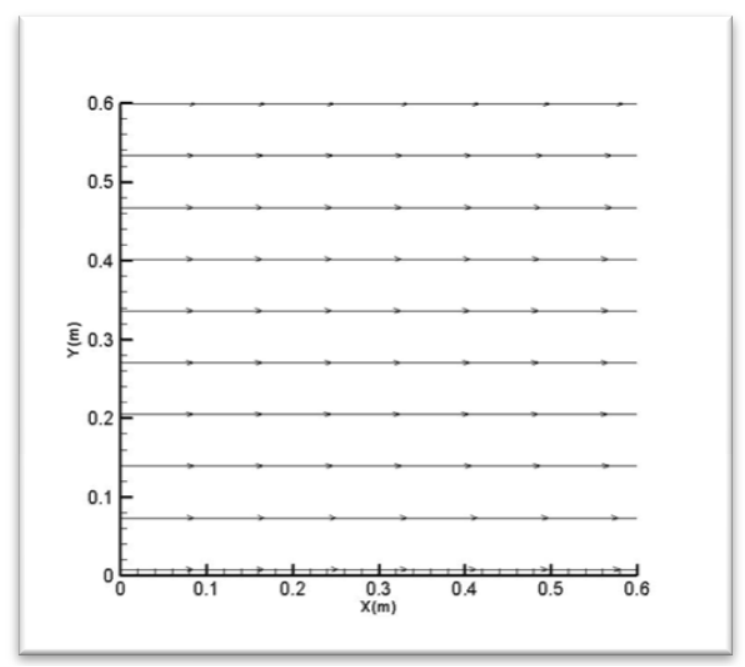

Streamline field

Figure 15. Pressure distribution and Streamline filed for one fracture cross two boundaries 


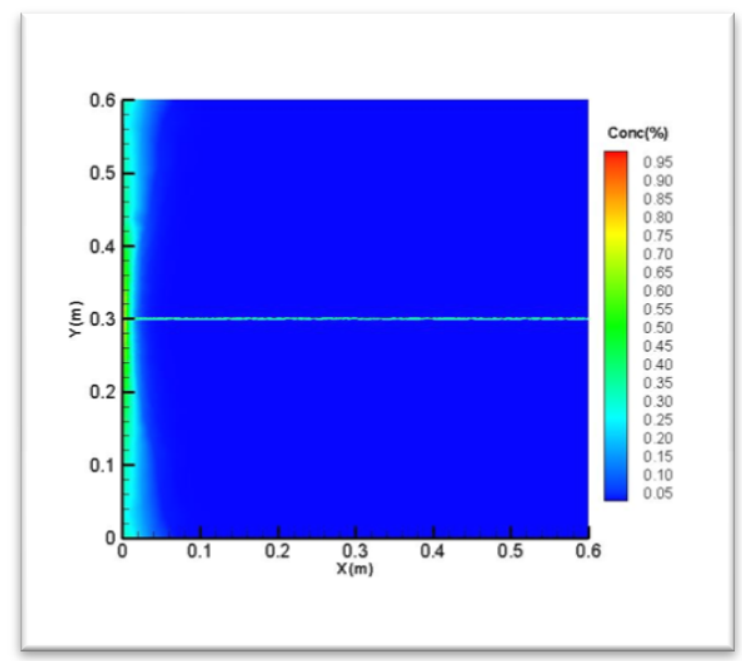

Concentration distribution at 100 year

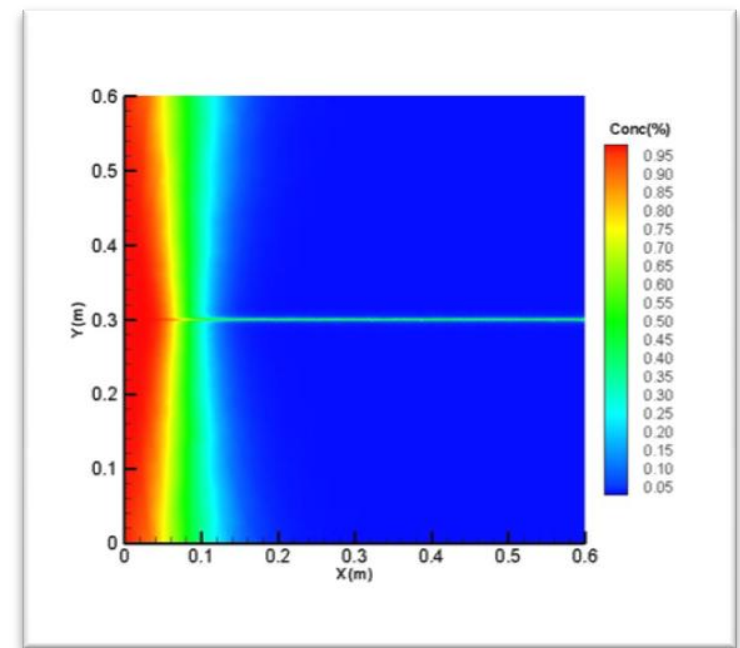

Concentration distribution at 1000 year

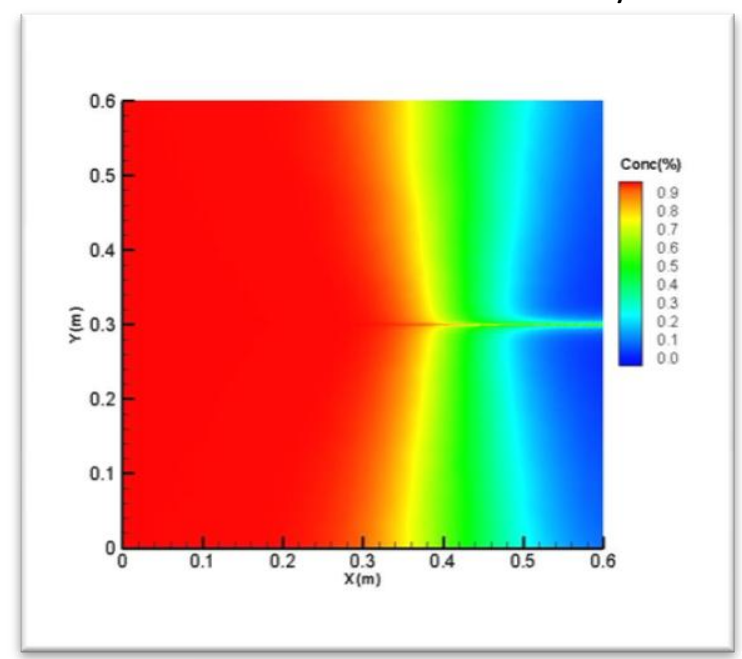

Concentration distribution at 5000 year

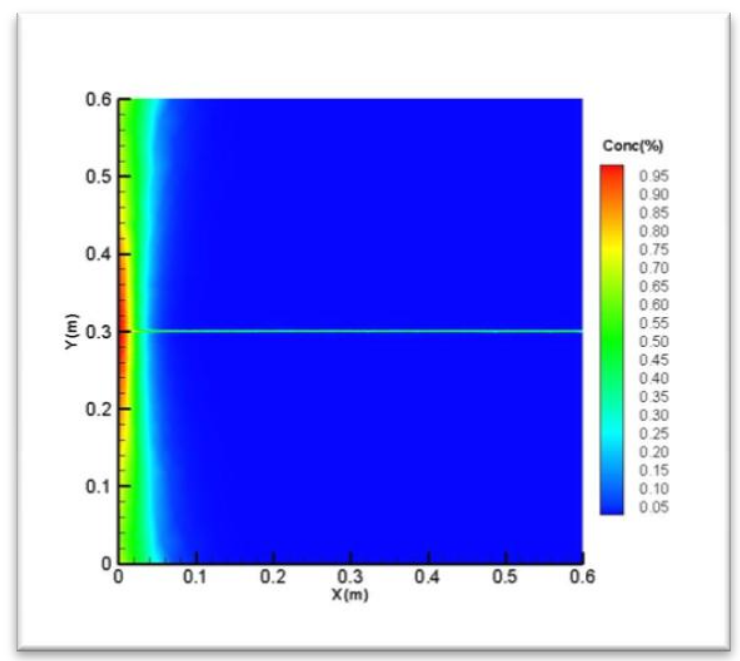

Concentration distribution at 300 year

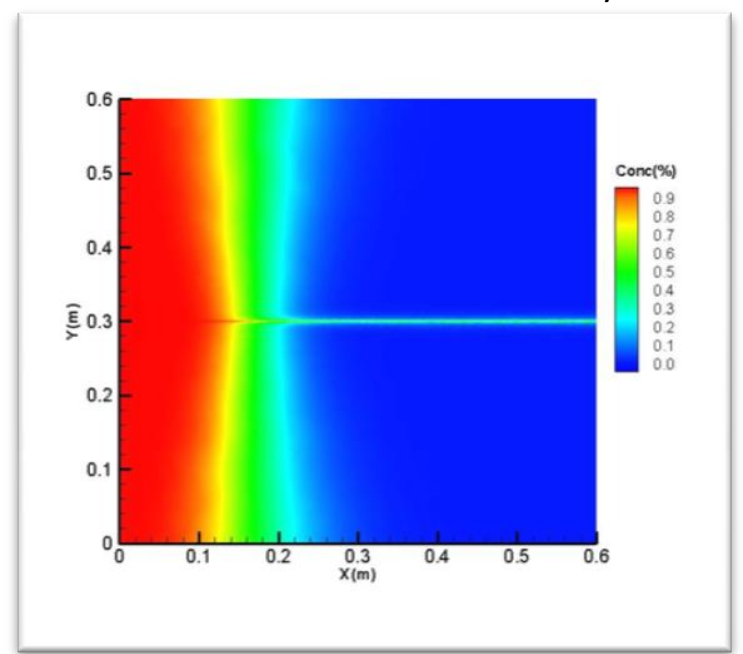

Concentration distribution at 2000 year

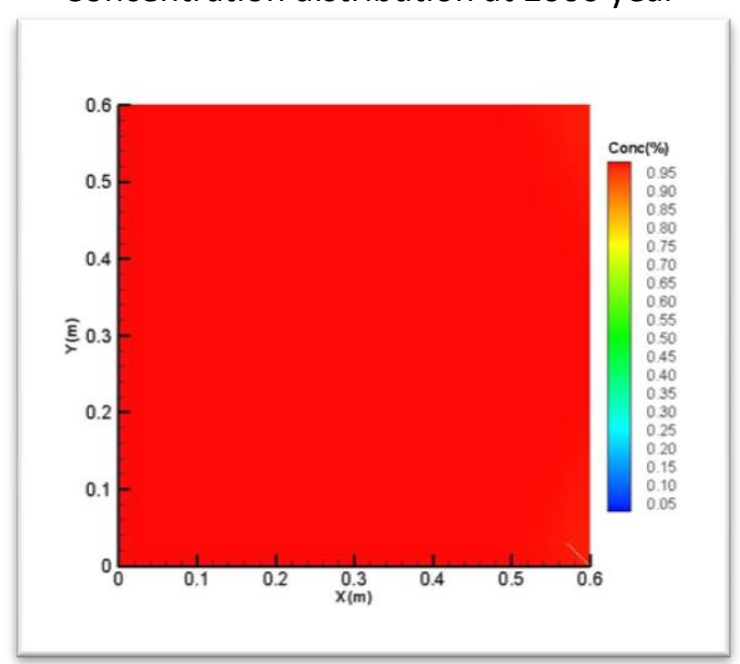

Concentration distribution at 10000 year Figure 16. Concentration at different time within ten thousand years for one fracture cross two boundaries 


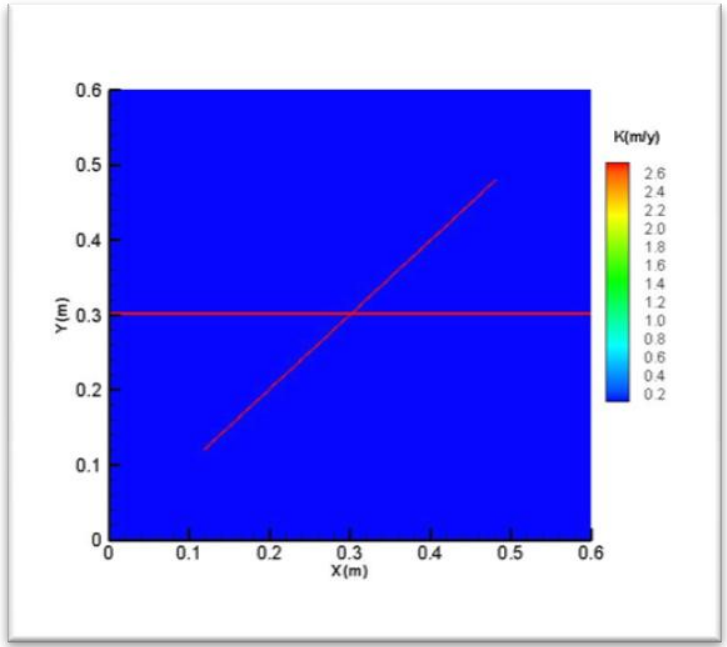

Enlarged fracture and conductivity distribution

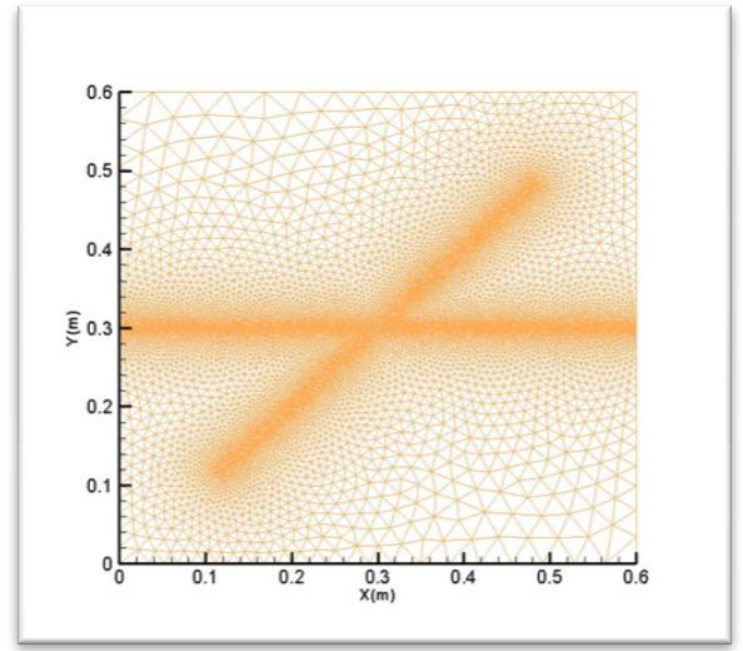

Triangular mesh for two crossed fracture

Figure 17. Fracture network for two crossed fractures extends to two boundaries

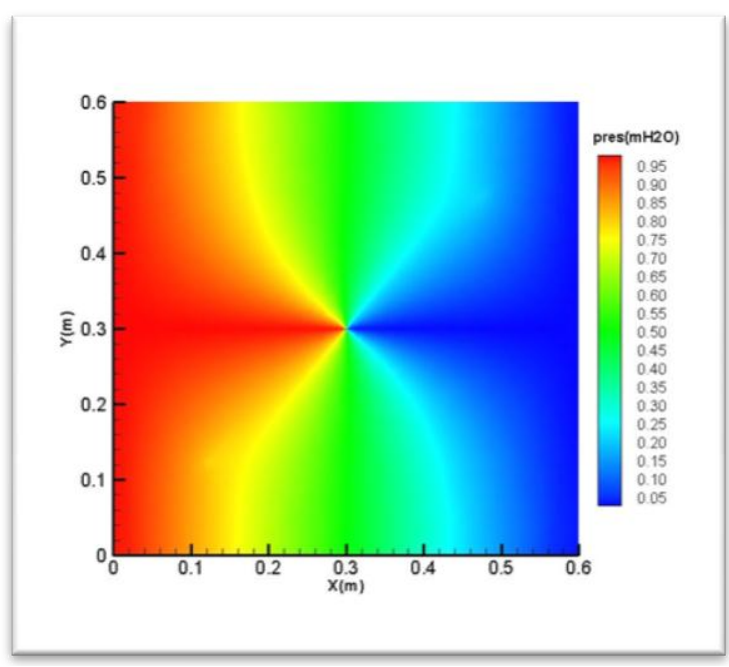

Pressure distribution

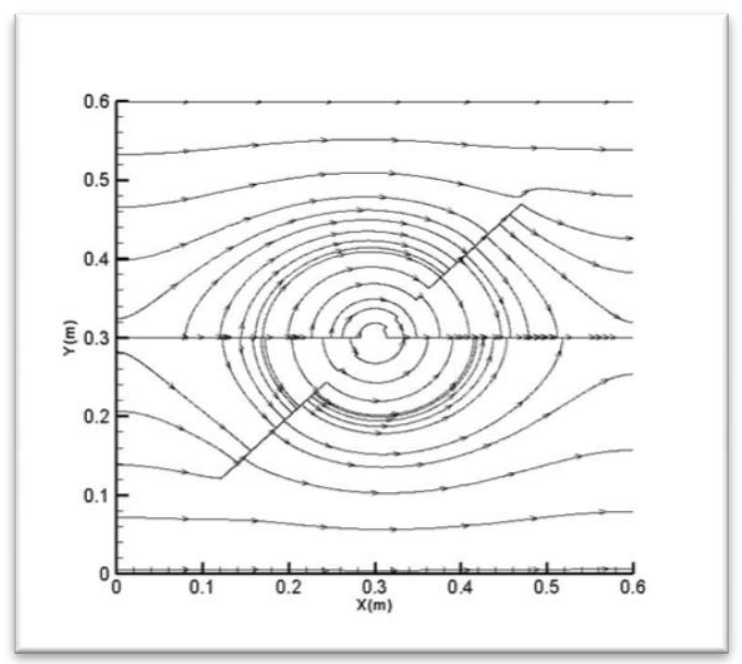

Streamline field

Figure 18. Pressure distribution and Streamline filed for two crossed fractures extends to two boundaries 


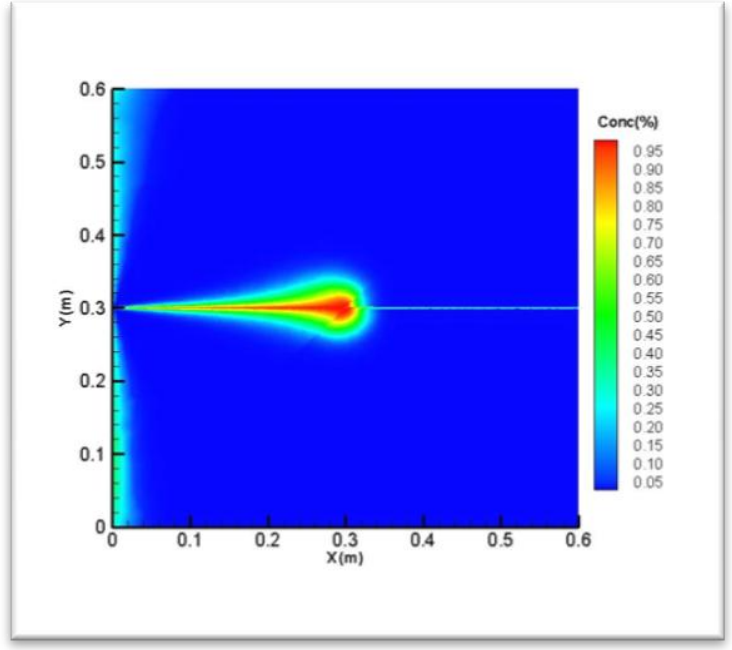

Concentration distribution at 100 year

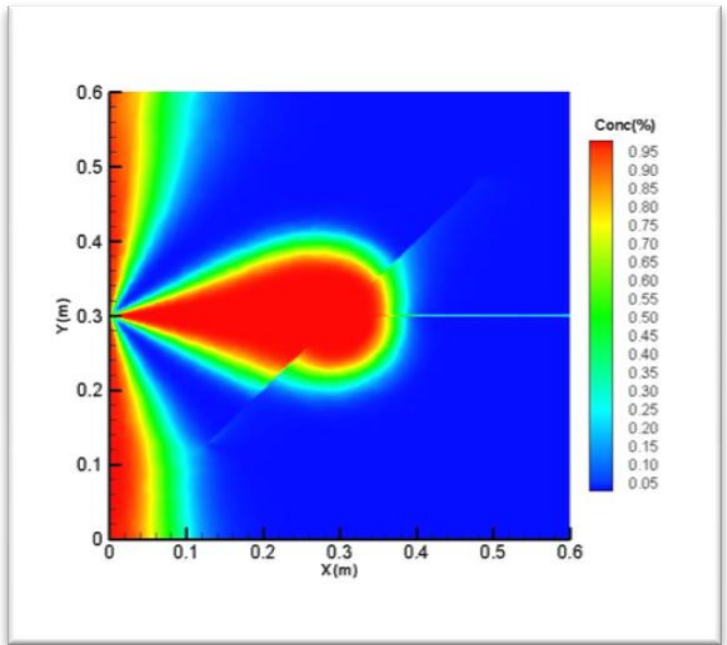

Concentration distribution at 1000 year

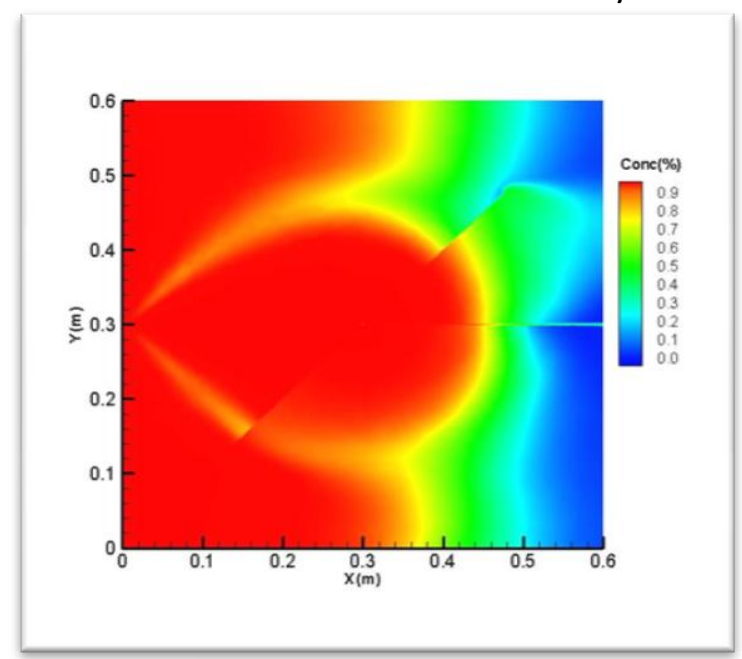

Concentration distribution at 5000 year

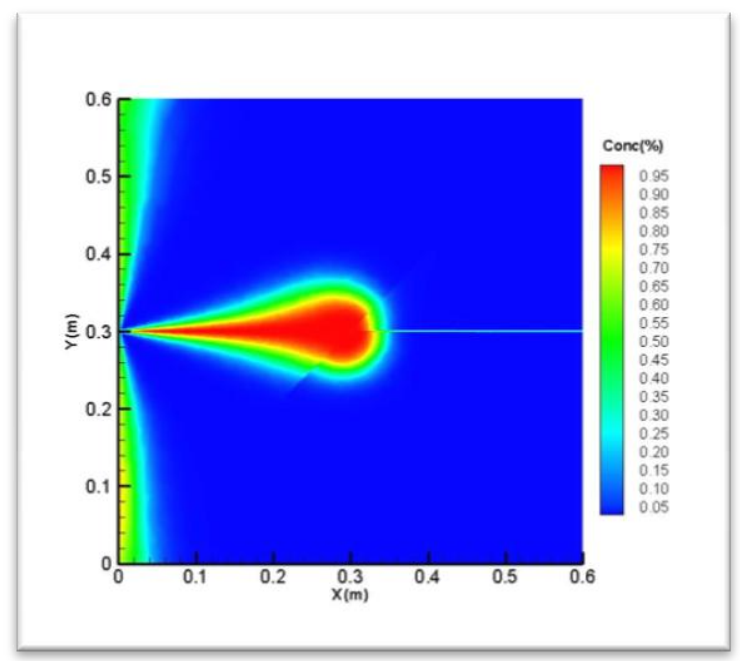

Concentration distribution at 300 year

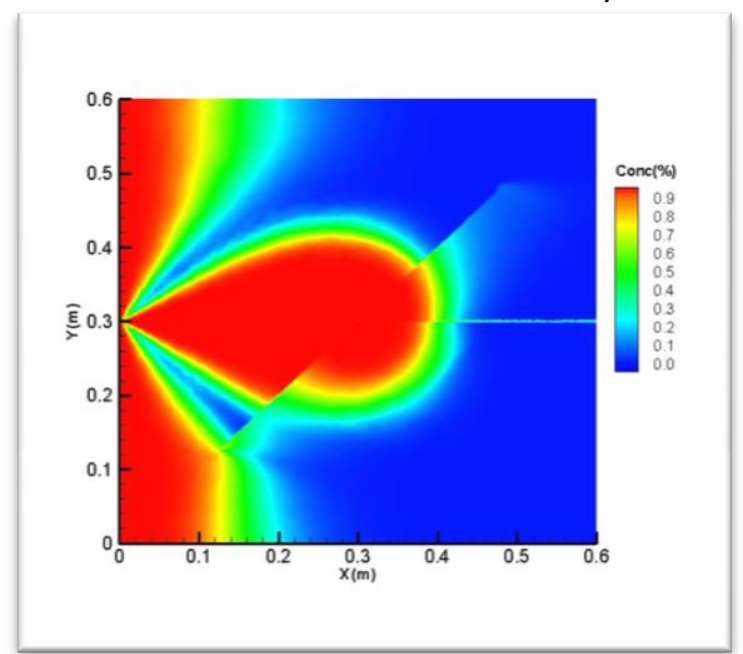

Concentration distribution at 2000 year

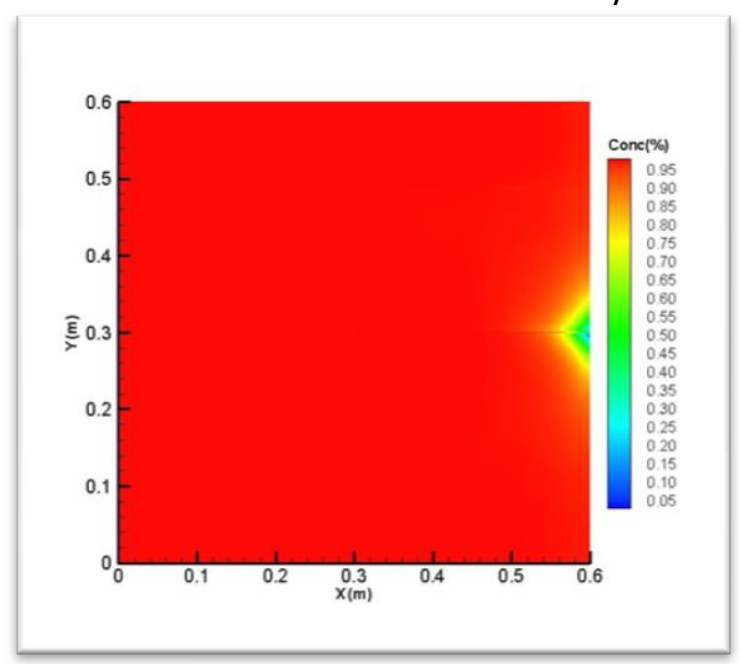

Concentration distribution at 10000 year Figure 19. Concentration at different time within ten thousand years for one fracture cross two boundaries 


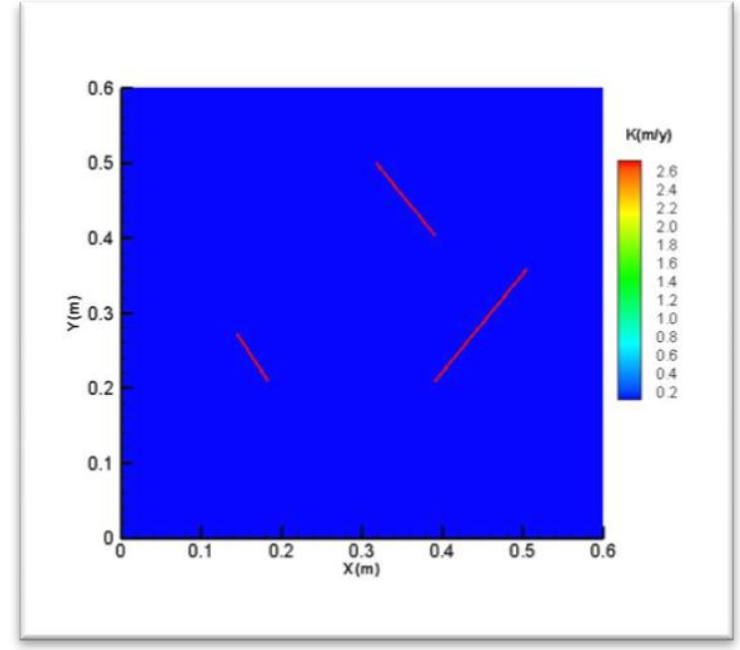

Enlarged fracture and conductivity distribution

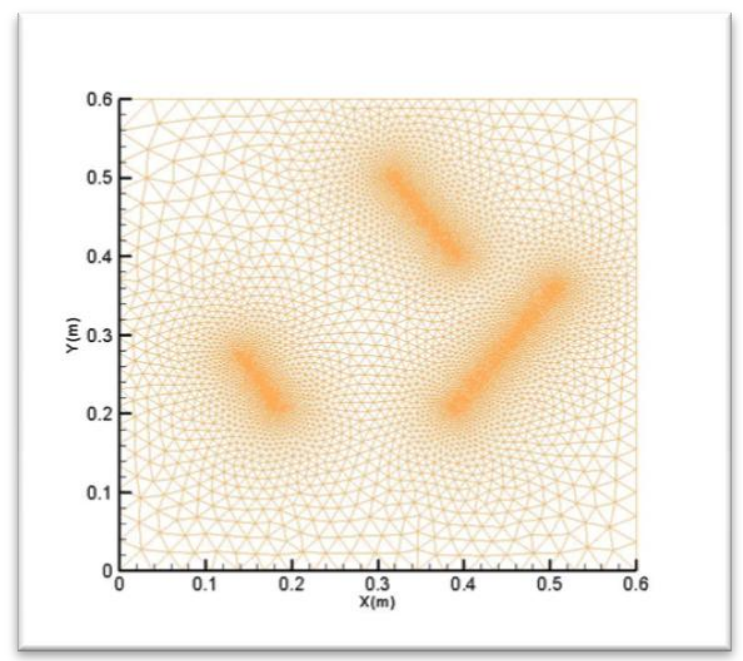

Triangular mesh for single fracture

Figure 20. Fracture network for 3 randomly generated fractures

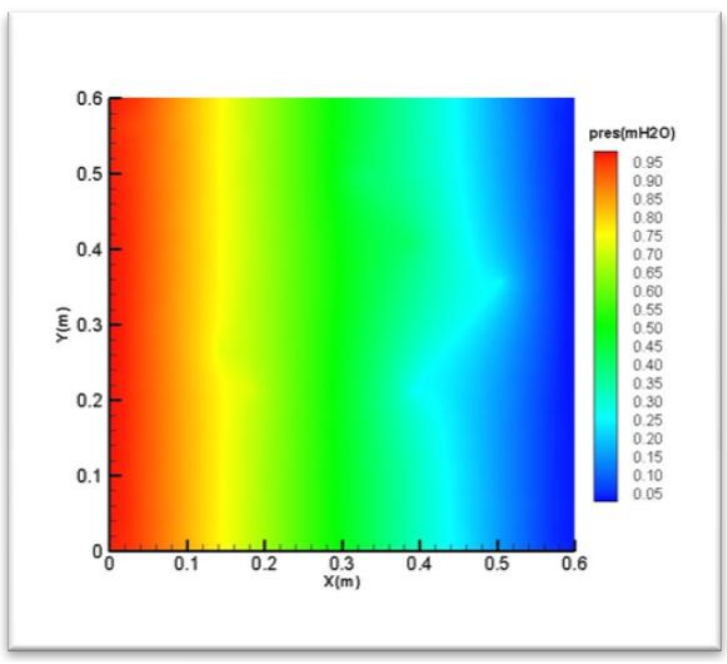

Pressure distribution

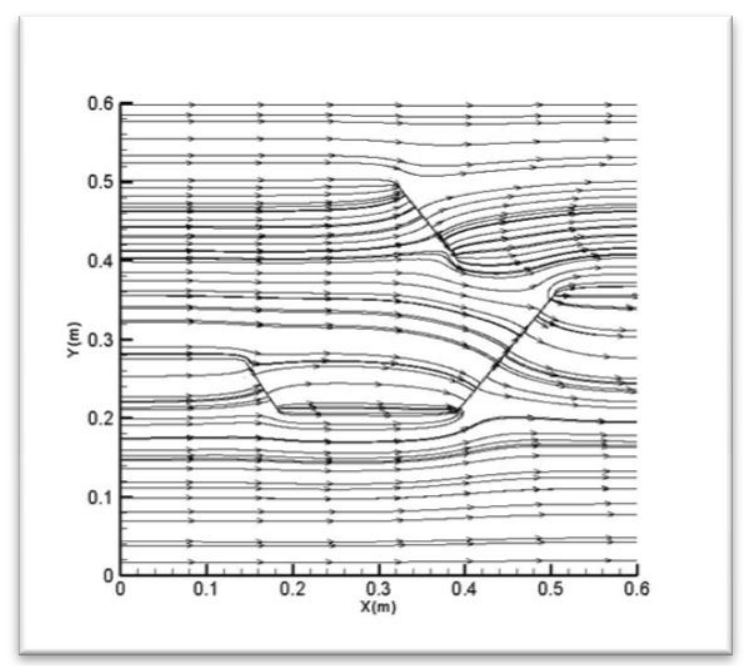

Streamline field

Figure 21. Pressure distribution and streamline filed for 3 randomly generated fractures 


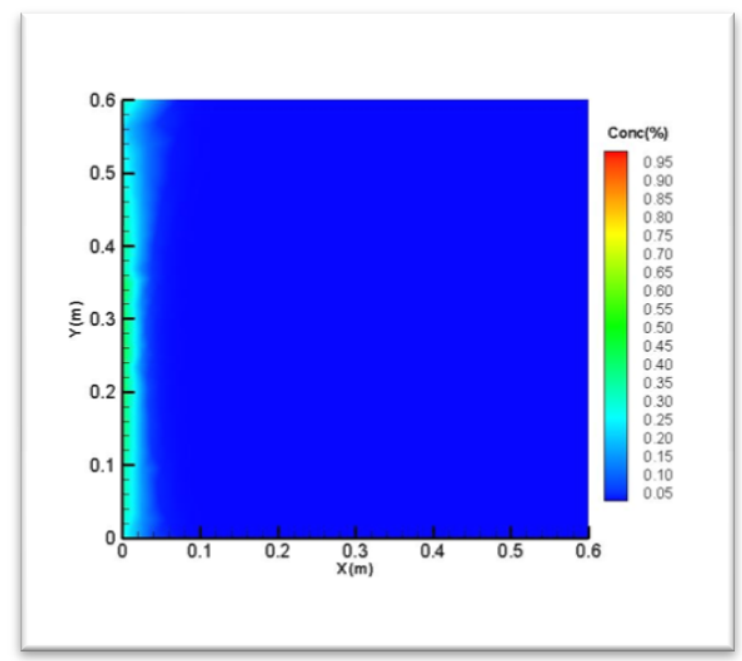

Concentration distribution at 100 year

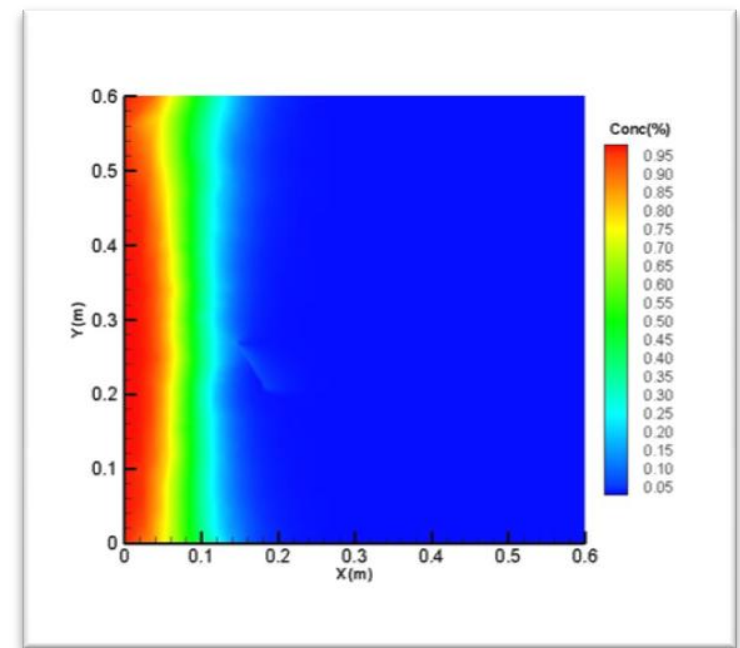

Concentration distribution at 1000 year

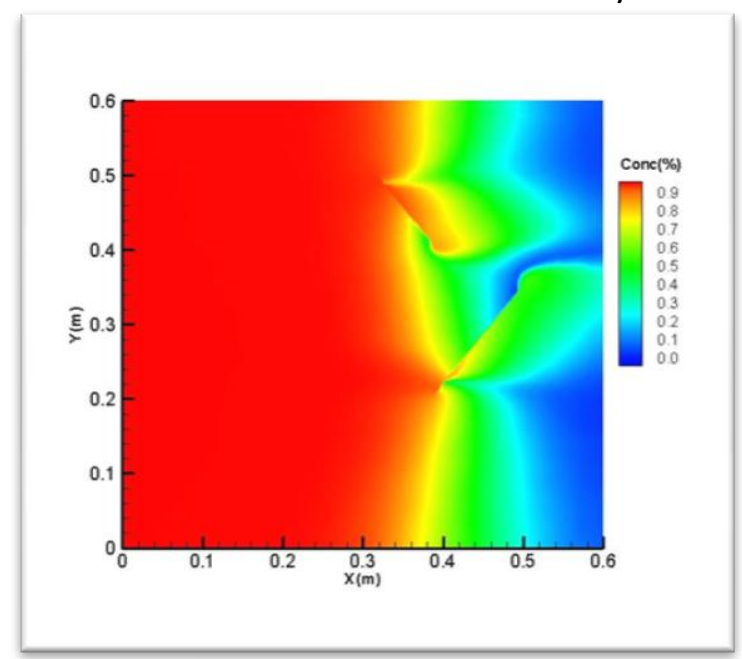

Concentration distribution at 5000 year

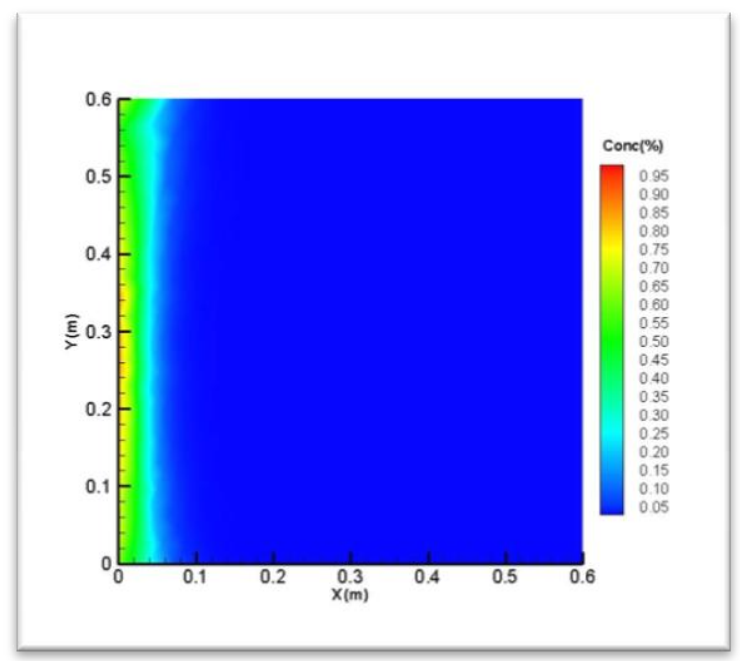

Concentration distribution at 300 year

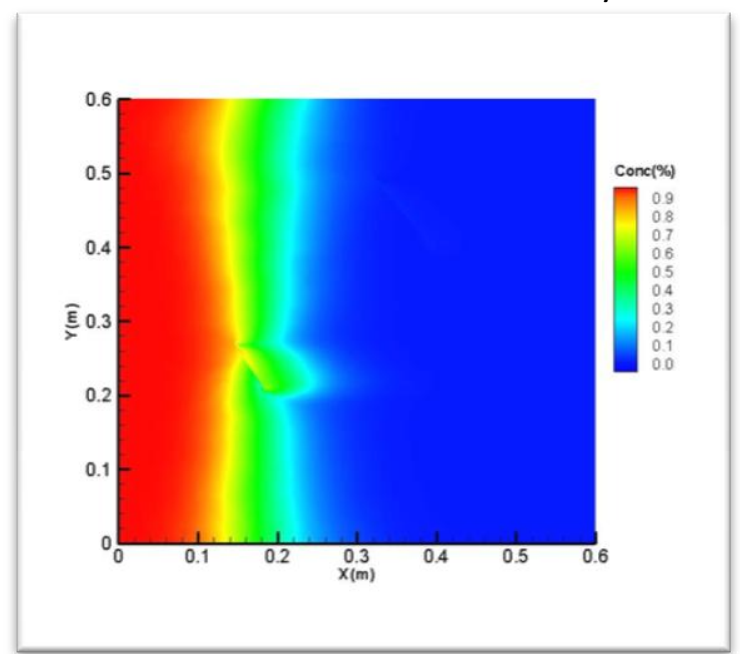

Concentration distribution at 2000 year

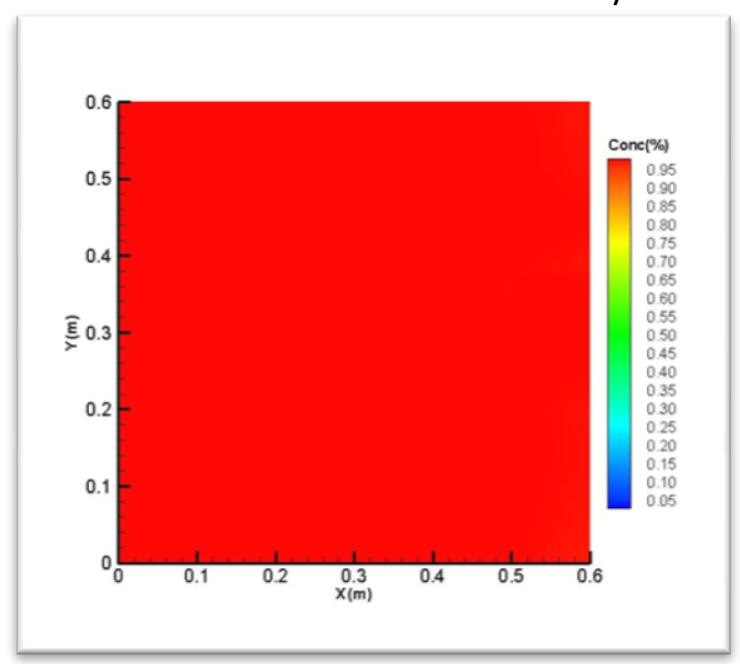

Concentration distribution at 10000 year Figure 22. Concentration at different time within ten thousand years for 3 randomly generated fractures 


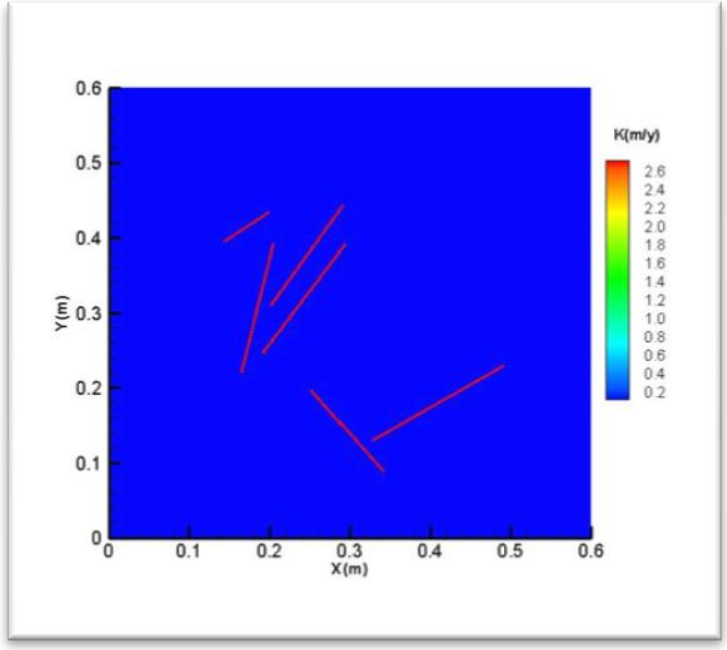

Enlarged fracture and conductivity distribution

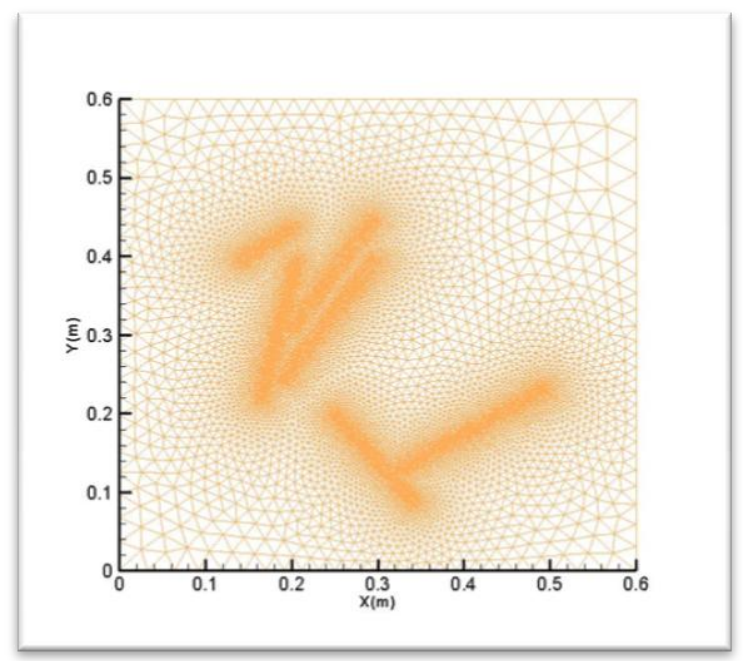

Triangular mesh for single fracture

Figure 23. Fracture network for 6 randomly generated fractures

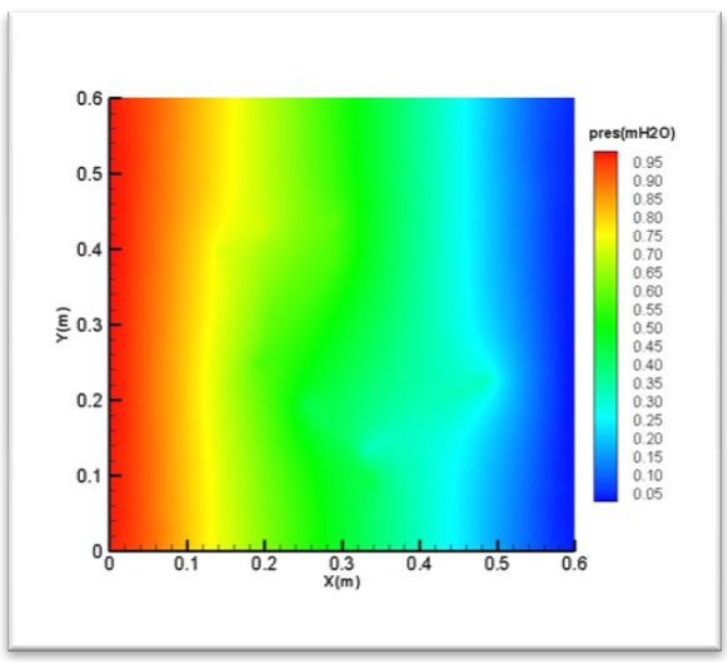

Pressure distribution

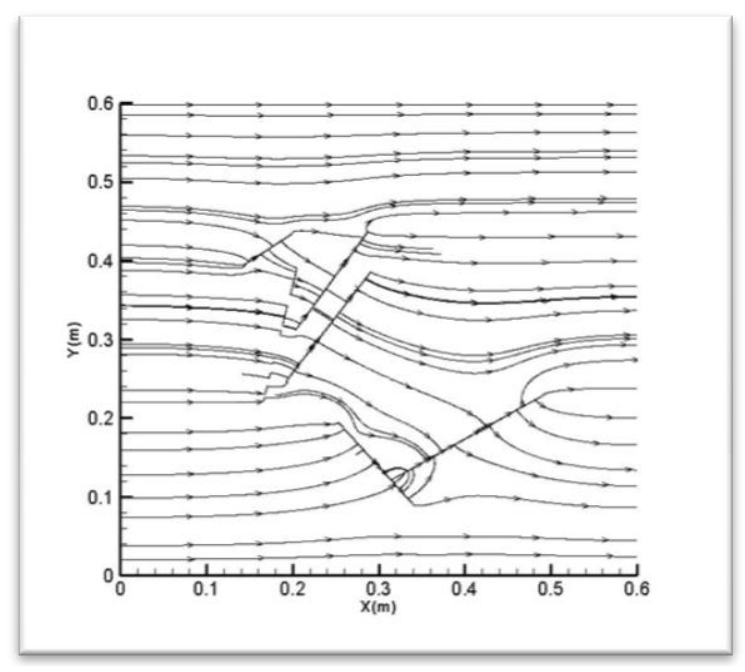

Streamline field

Figure 24. Pressure distribution and streamline filed for 6 randomly generated fractures 


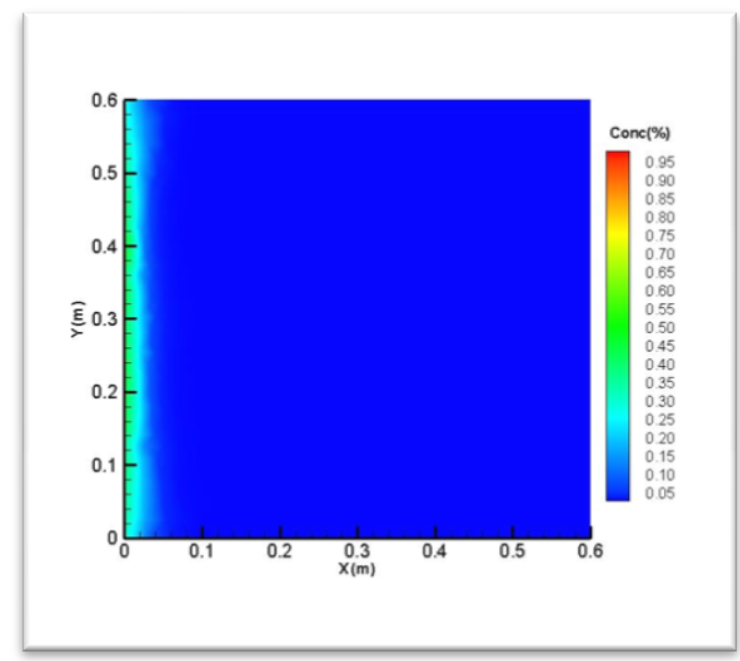

Concentration distribution at 100 year

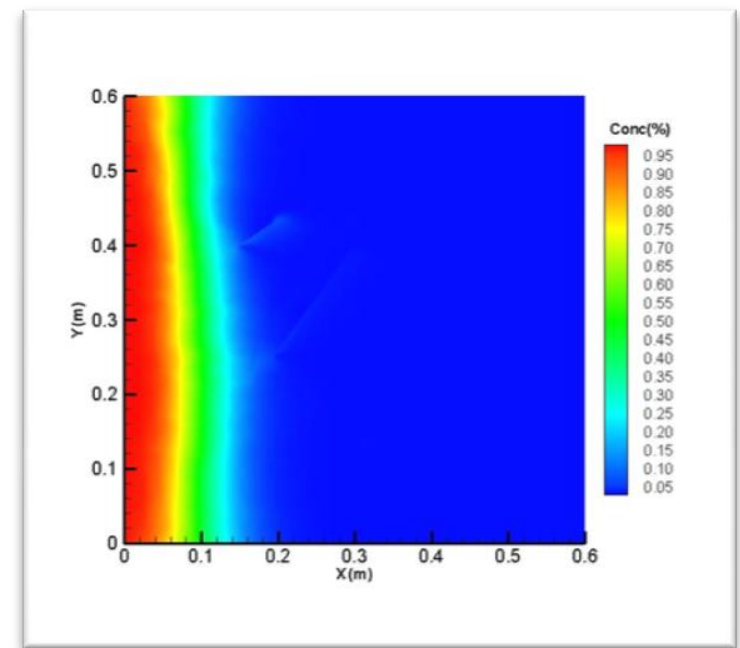

Concentration distribution at 1000 year

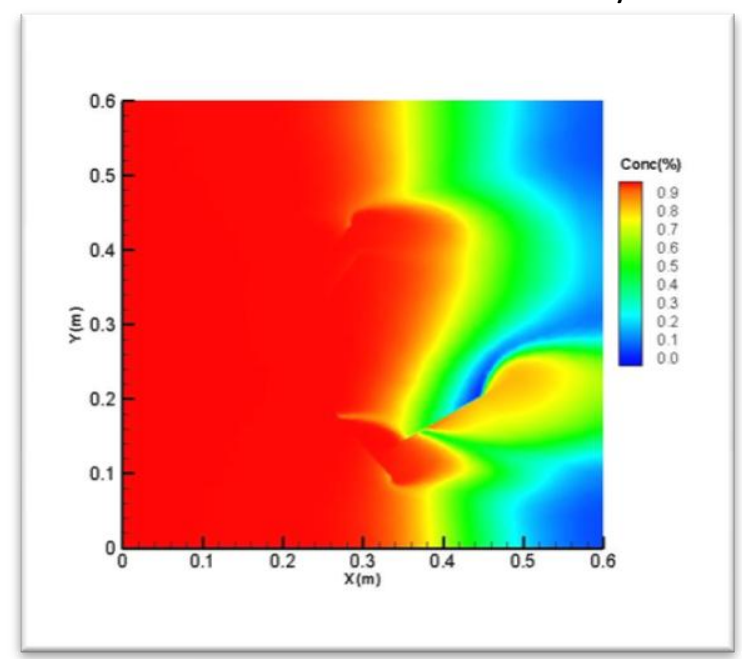

Concentration distribution at 5000 year

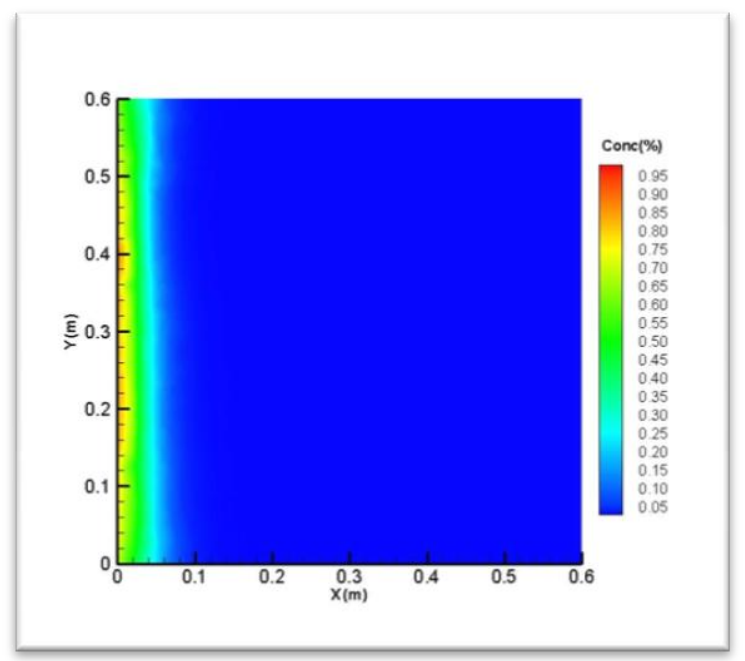

Concentration distribution at 300 year

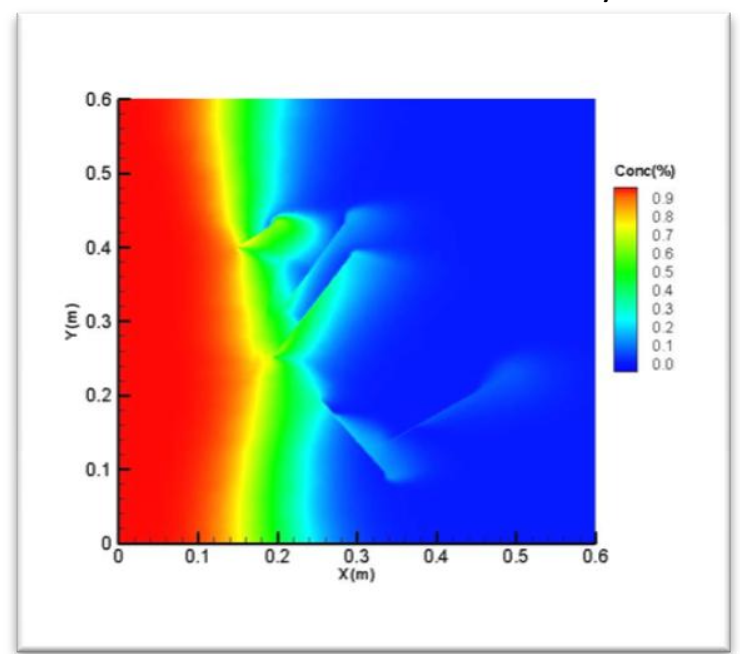

Concentration distribution at 2000 year

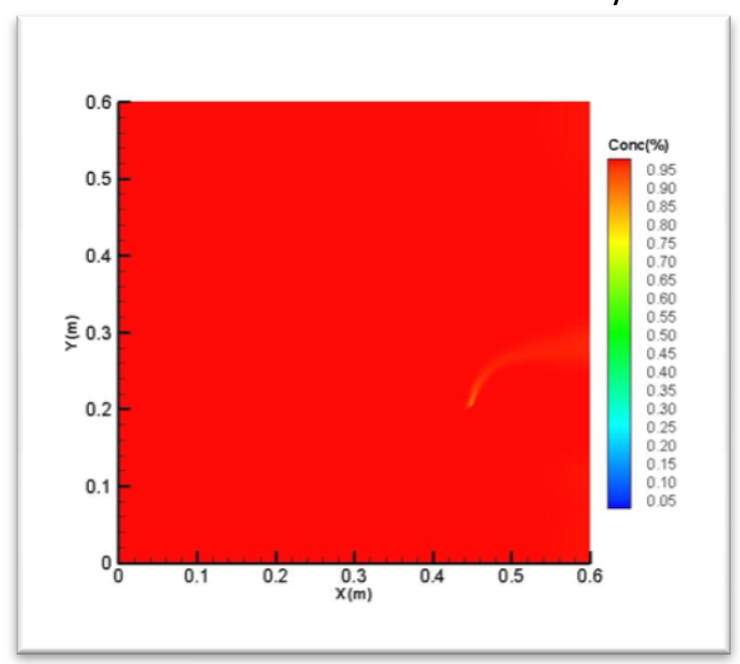

Concentration distribution at 10000 year Figure 25. Concentration at different time within ten thousand years for 6 randomly generated fractures 


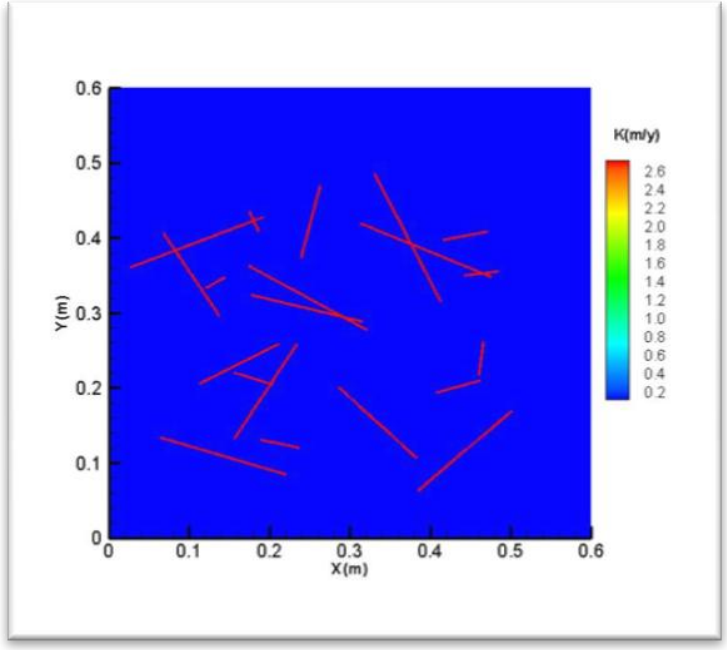

Enlarged fracture and conductivity distribution

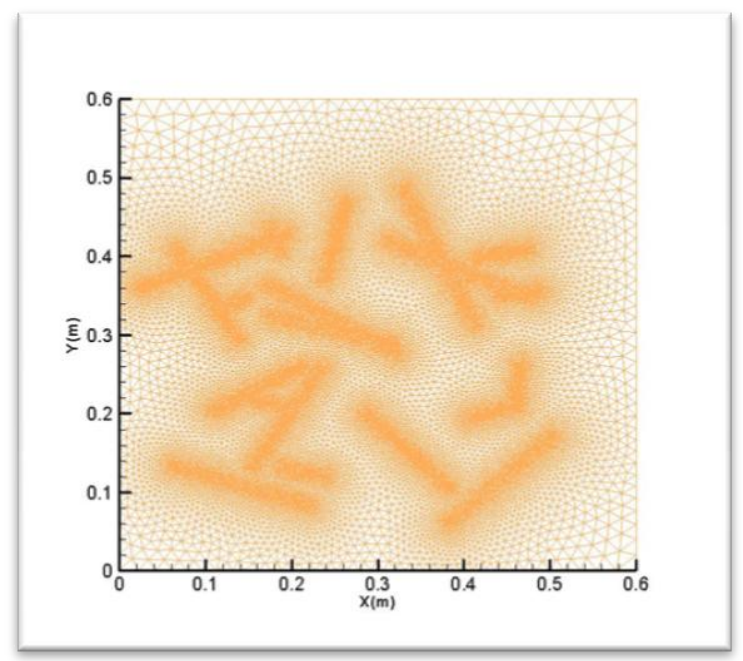

Triangular mesh for single fracture

Figure 26. Fracture network for 20 randomly generated fractures

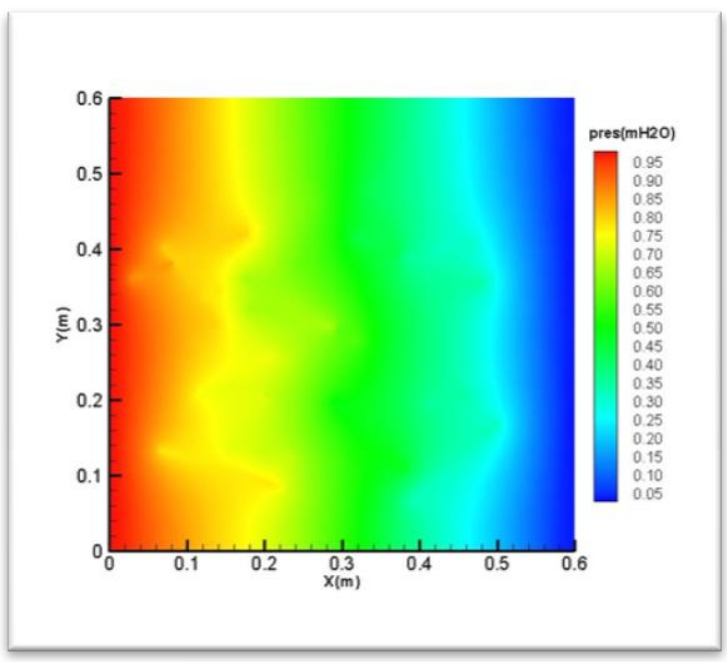

Pressure distribution

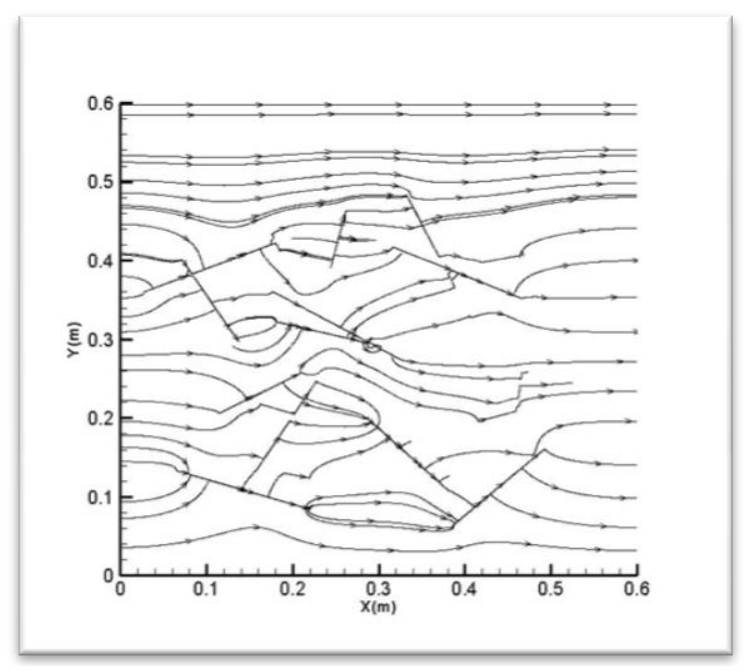

Streamline field

Figure 27. Pressure distribution and streamline filed for 20 randomly generated fractures 


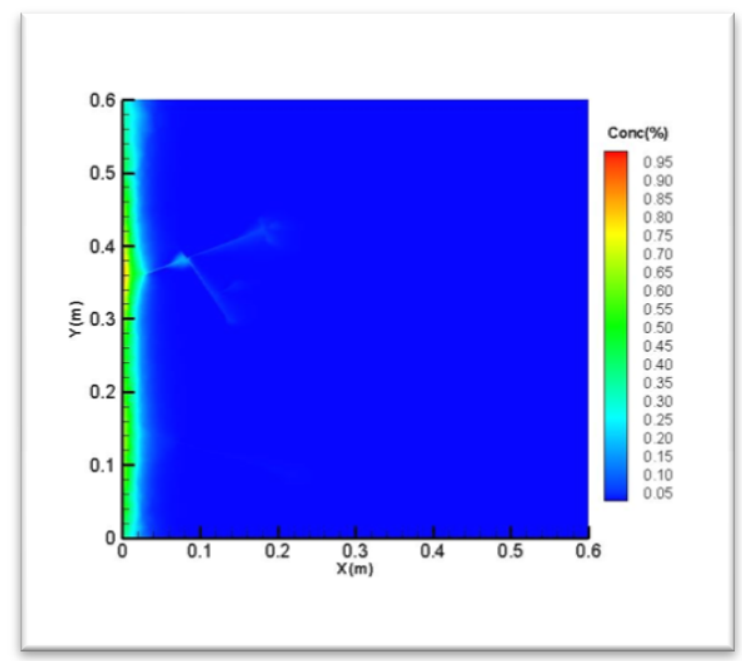

Concentration distribution at 100 year

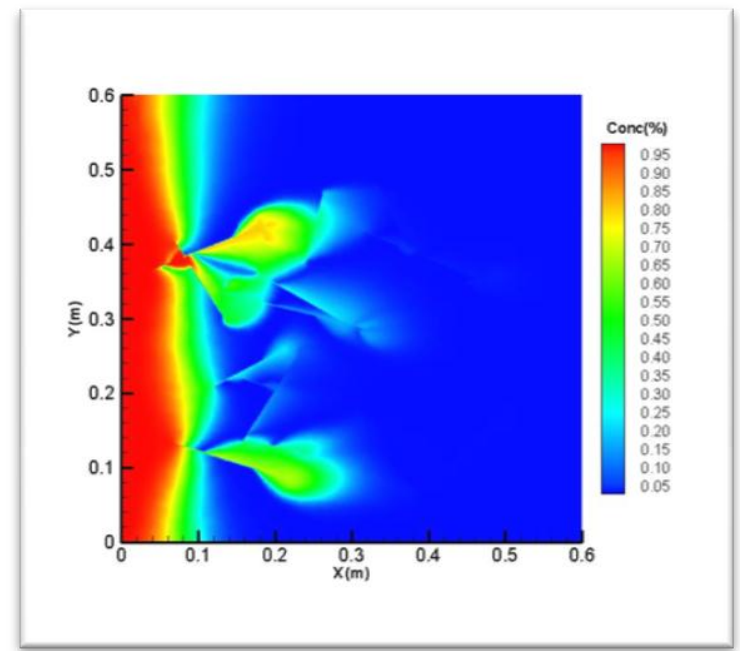

Concentration distribution at 1000 year

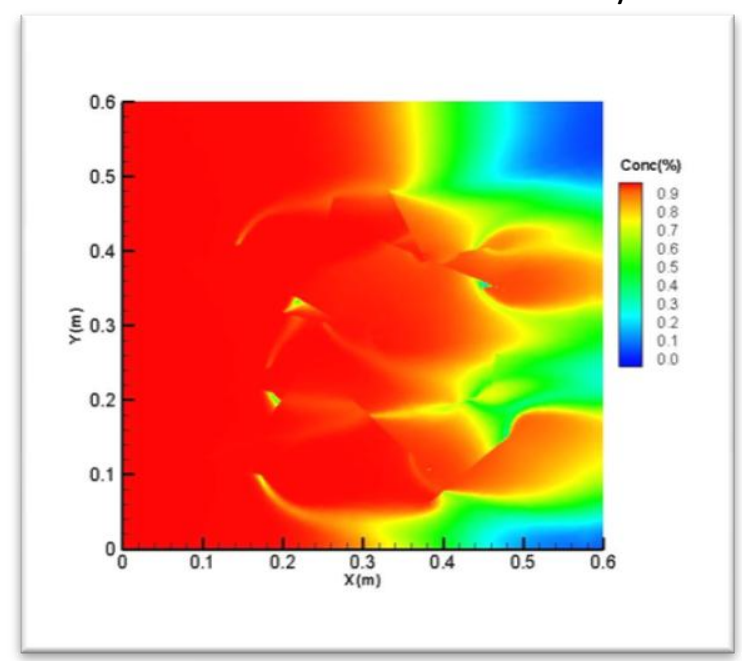

Concentration distribution at 5000 year

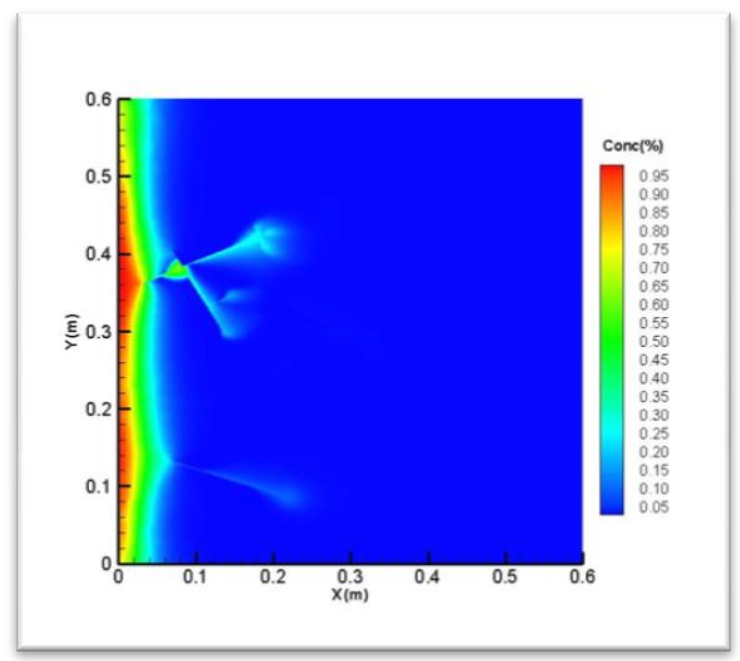

Concentration distribution at 300 year

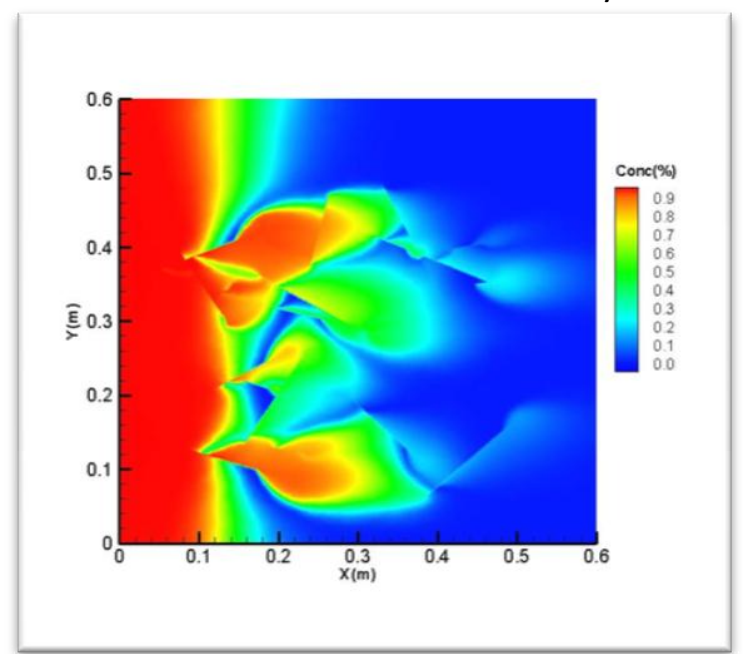

Concentration distribution at 2000 year

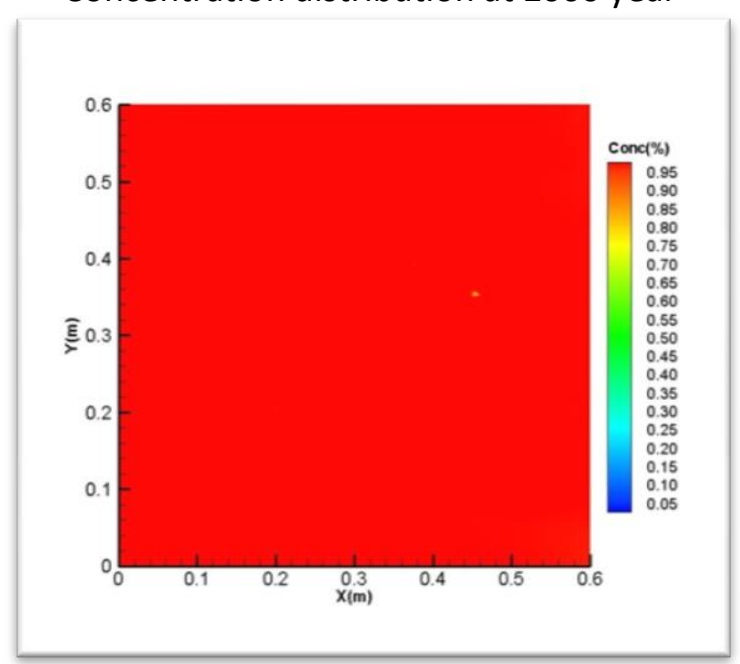

Concentration distribution at 10000 year

Figure 28. Concentration at different time within ten thousand years for 30 randomly generated fractures 


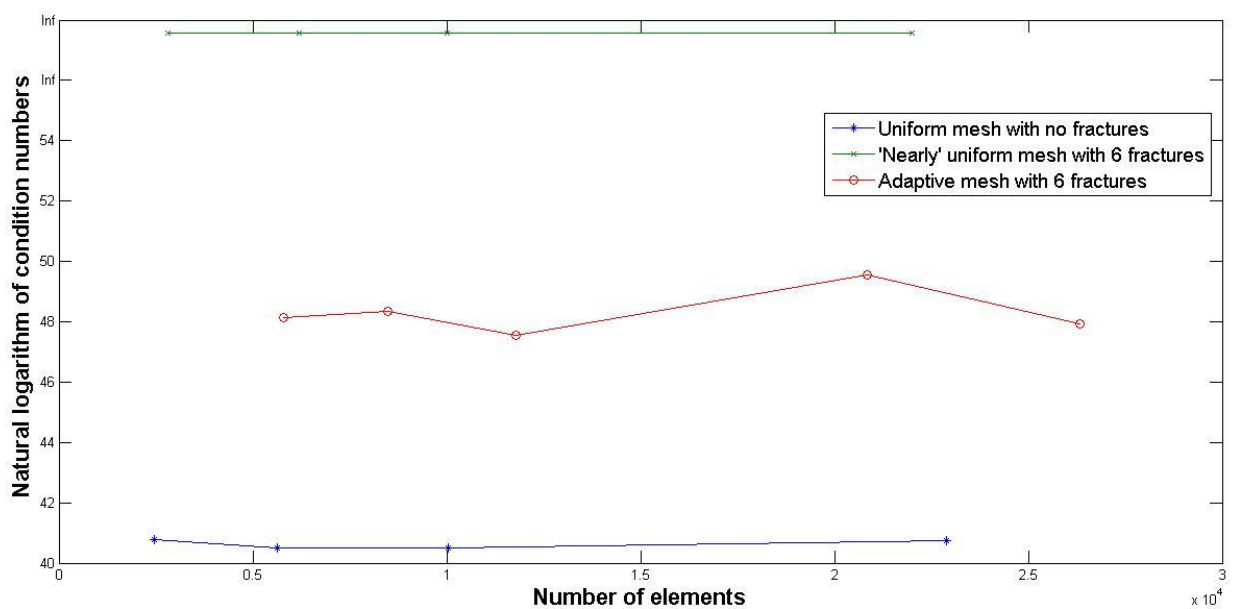

Figure 29. Natural logarithm of condition numbers of $A_{T}$ vs Numbers of elements

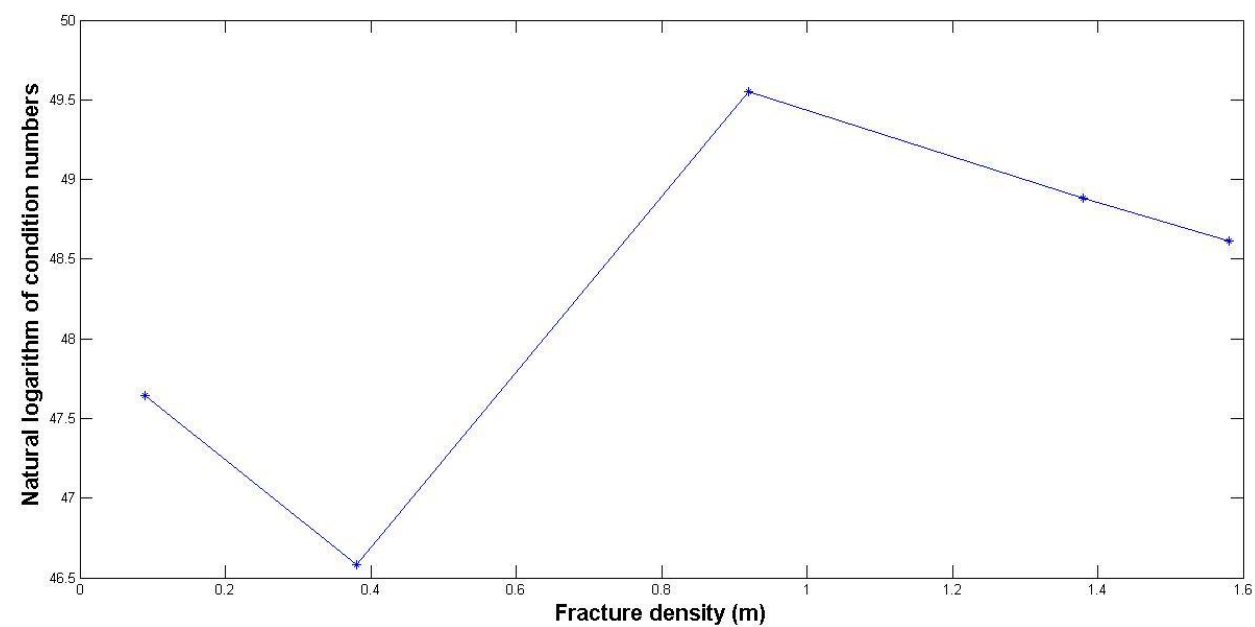

Figure 30. Natural logarithm of condition numbers of $A_{T}$ vs Fracture density 


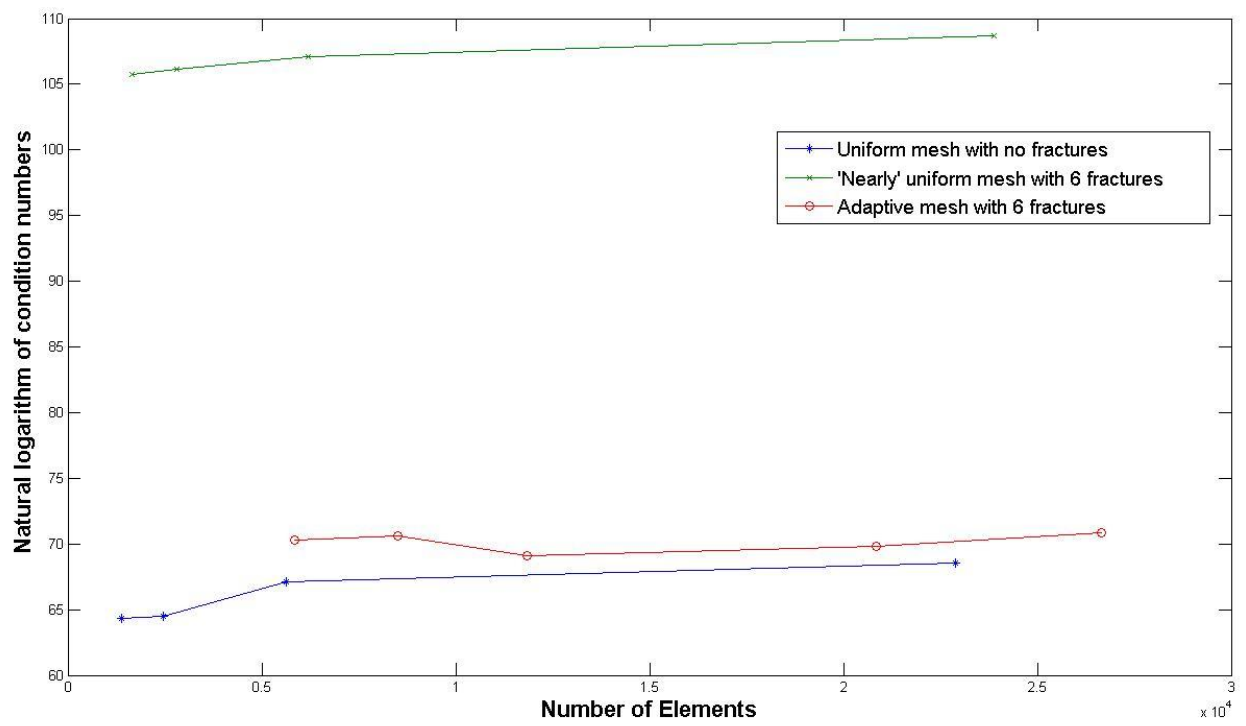

Figure 31. Natural logarithm of condition numbers of A vs Numbers of elements

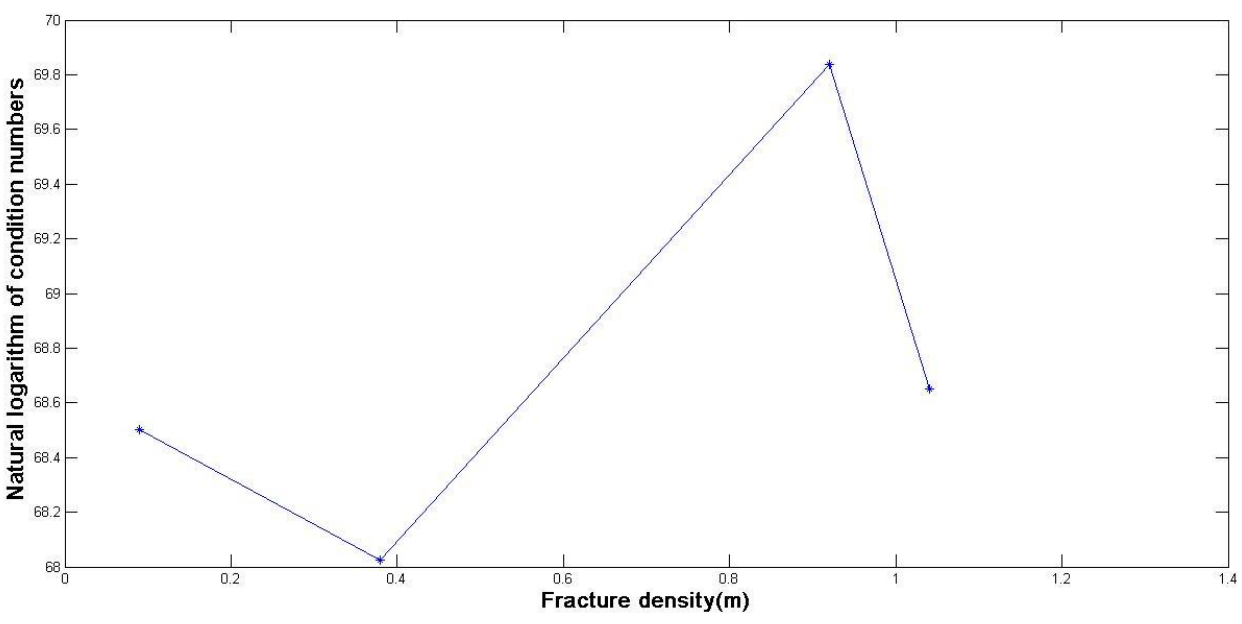

Figure 32. Natural logarithm of condition numbers of A vs Fracture density 


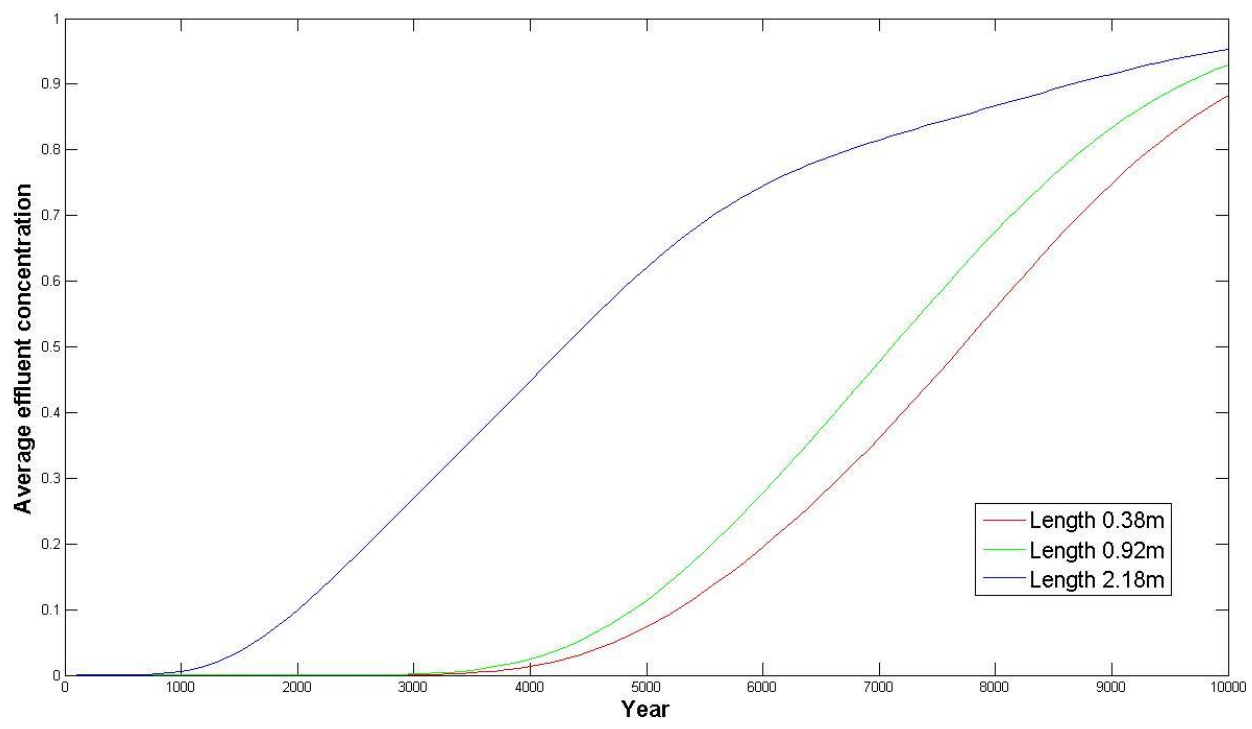

Figure 33. Average effluent concentration on outflow boundary vs time with different fracture density

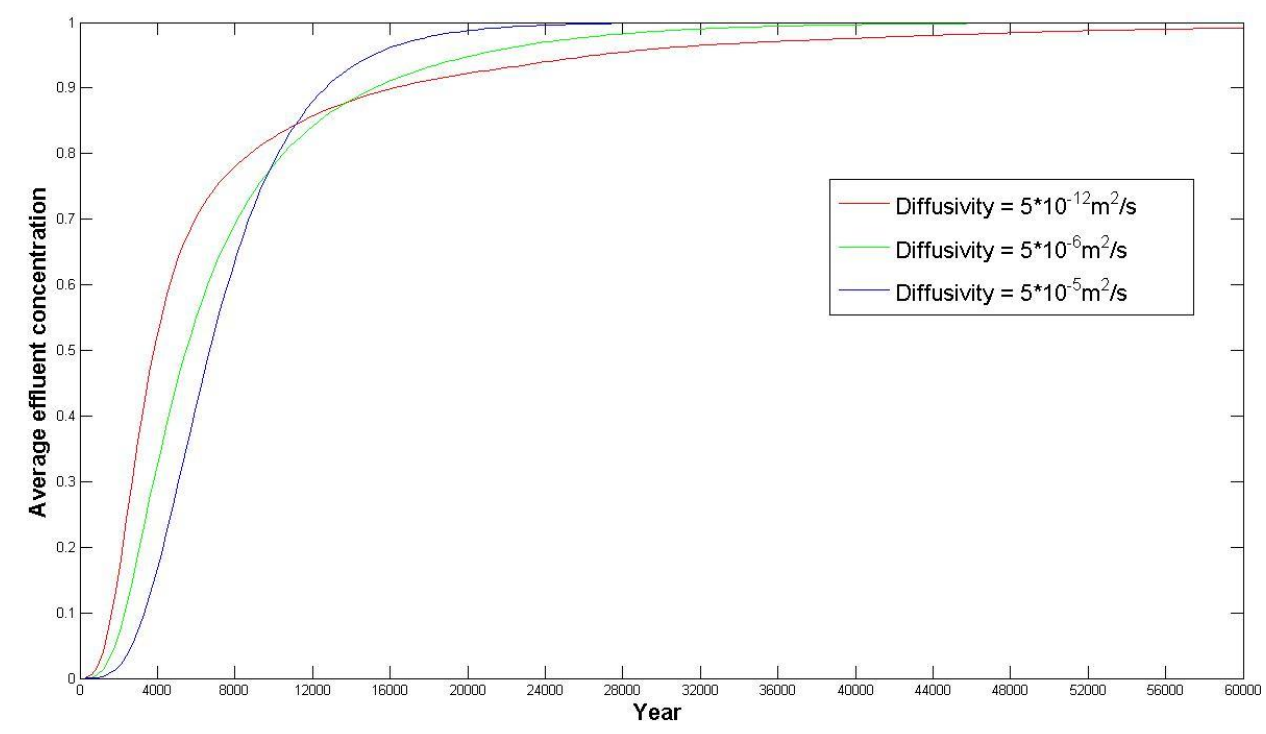

Figure 34. Average effluent concentration on outflow boundary vs time with different diffusivity 


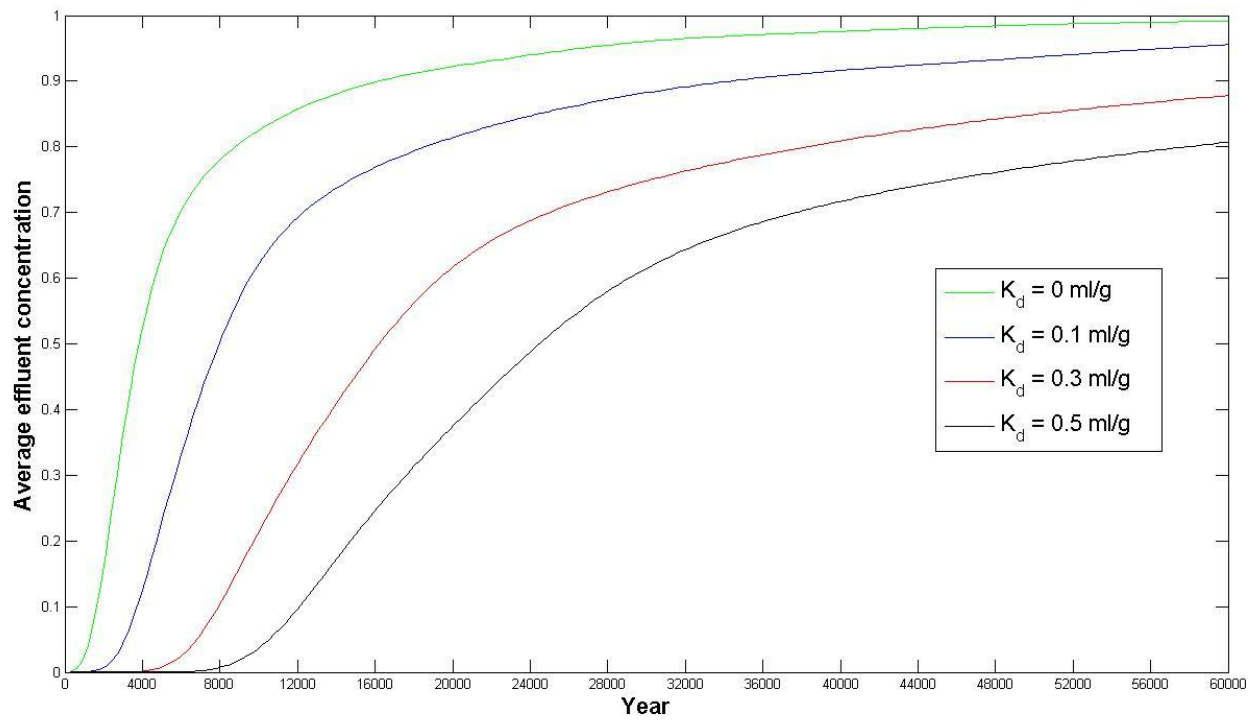

Figure 35. Average effluent concentration on outflow boundary vs time with different $K_{d}$ value

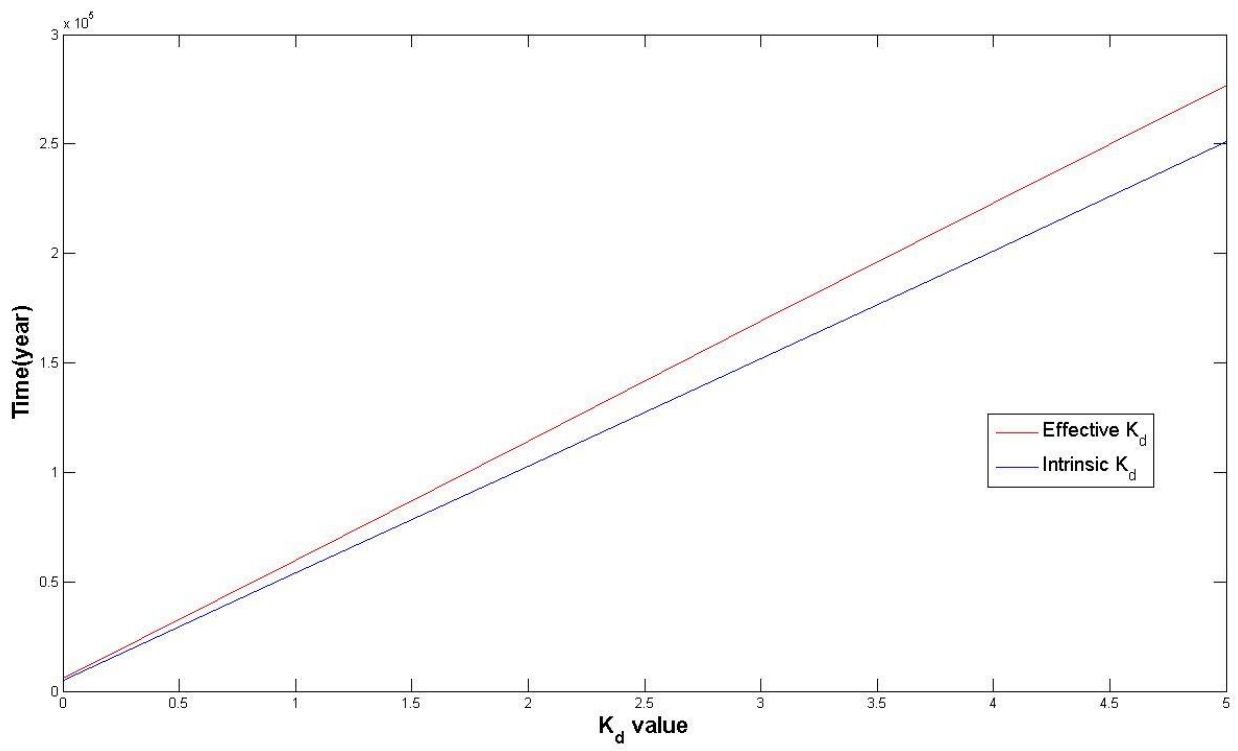

Figure 36. Intrinsic and effective $K_{d}$ value vs time 


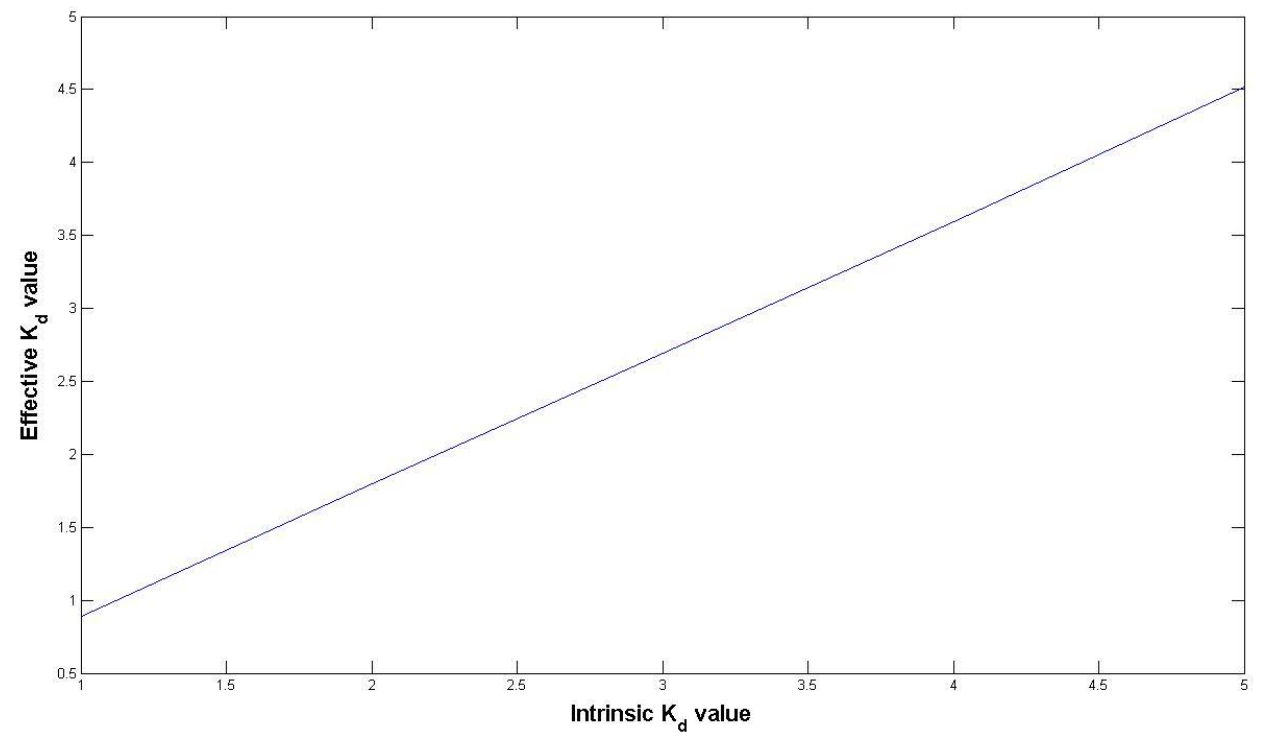

Figure 37. Intrinsic $K_{d}$ value vs effective $K_{d}$ value 